\title{
The extraction of Campbell diagrams from the dynamical system representation of a foil-air bearing rotor model
}

DOI:

10.1016/j.ymssp.2019.04.018

\section{Document Version}

Accepted author manuscript

Link to publication record in Manchester Research Explorer

\section{Citation for published version (APA):}

Bonello, P. (2019). The extraction of Campbell diagrams from the dynamical system representation of a foil-air bearing rotor model. Mechanical Systems and Signal Processing, 129, 502-530.

https://doi.org/10.1016/j.ymssp.2019.04.018

\section{Published in:}

Mechanical Systems and Signal Processing

\section{Citing this paper}

Please note that where the full-text provided on Manchester Research Explorer is the Author Accepted Manuscript or Proof version this may differ from the final Published version. If citing, it is advised that you check and use the publisher's definitive version.

\section{General rights}

Copyright and moral rights for the publications made accessible in the Research Explorer are retained by the authors and/or other copyright owners and it is a condition of accessing publications that users recognise and abide by the legal requirements associated with these rights.

\section{Takedown policy}

If you believe that this document breaches copyright please refer to the University of Manchester's Takedown Procedures [http://man.ac.uk/04Y6Bo] or contact uml.scholarlycommunications@manchester.ac.uk providing relevant details, so we can investigate your claim.

\section{OPEN ACCESS}




\title{
The Extraction of Campbell Diagrams from the
}

\section{Dynamical System Representation of a Foil-Air Bearing Rotor Model}

\author{
Philip Bonello
}

\author{
School of Mechanical, Aerospace and Civil Engineering, \\ University of Manchester,
}

Sackville Street, Manchester M13 9PL, United Kingdom

Corresponding author: P. Bonello

Email: philip.bonello@manchester.ac.uk 


\title{
The Extraction of Campbell Diagrams from the
}

\section{Dynamical System Representation of a Foil-Air Bearing Rotor Model}

P Bonello,

School of Mechanical, Aerospace and Civil Engineering, University of Manchester, United Kingdom

\begin{abstract}
Campbell diagrams describe the variation with speed of the modes of low amplitude (linear) free vibration of a rotordynamic system about its static equilibrium condition, and are an important tool that aids the interpretation of the nonlinear response. However, Campbell diagrams for foil-air bearing (FAB) rotor systems are currently derived using the linear force coefficients (FC) method and individual modes have not been verified by transient nonlinear dynamic analysis (TNDA) at low amplitudes. In fact, significant discrepancies between FC and TNDA predictions for the stability of the leading (i.e. least damped) mode have been reported. This paper establishes for the first time a method for deriving Campbell diagrams of a generic FAB-rotor model that avoids the FC method. It is based on an eigenvalue analysis of the Jacobian matrix of the dynamical system. Through suitable scaling and the application of appropriate criteria, the multitude of eigenvectors of the Jacobian can be filtered to extract the relevant modes. Each mode is verified using TNDA with mode-specific initial conditions derived from the Campbell diagram analysis. The methodology is successfully tested on three cases in the literature, which respectively illustrate its applicability to complex bearing types (3-pad), complex foil models, and flexible rotors.
\end{abstract}

Keywords: Foil-air bearings; dynamical systems; nonlinear analysis; linearisation; Campbell diagrams

\section{Abbreviations: FAB $^{1}$; SESA $^{2} ;$ TNDA $^{3} ;$ OIS $^{4} ;$ FLE CTE $^{5} ;$ SEFM $^{6} ;$ FFSMM $^{7} ;$ FC $^{8} ;$ FD $^{9} ;$ FE $^{10}$}

\footnotetext{
${ }^{1}$ Foil-air bearing

${ }^{2}$ Static equilibrium stability analysis

${ }^{3}$ Transient nonlinear dynamic analysis

${ }^{4}$ Onset of instability speed

${ }^{5}$ Free leading edge/ clamped trailing edge

${ }^{6}$ Simple equivalent foundation model

${ }^{7}$ Full foil structure modal model

${ }^{8}$ Force coefficients

${ }^{9}$ Finite difference

${ }^{10}$ Finite element
} 


\section{INTRODUCTION}

In the predictive dynamic analysis of rotating machinery running in nonlinear bearings, the efficient simulation and interpretation of the various aspects of the dynamic response, whether forced or selfexcited, linear or nonlinear, necessitate a suite of computational tools that produces consistent results. While this has not been a problem for machinery running in incompressible fluid bearings (e.g. oil bearings), it continues to be a problem for foil-air bearing (FAB) rotor systems, as elucidated in the literature review below. This is a significant omission, since FABs are the key to the rapidly expanding green technology of oil-free turbomachinery [1]. The root of this problem is the reluctance, driven by the complexities involved [1], to base all aspects of the dynamic analysis of the generic FAB-rotor model on its dynamical system representation.

In nonlinear dynamics theory, the dynamical system representation of a nonlinear model follows the generic state space format (first order ordinary differential equations system) [2]

$$
\mathbf{s}^{\prime}=\chi(\tau, \mathbf{s})
$$

where ()$^{\prime}$ denotes differentiation with respect to the time variable $\tau, \mathbf{s}$ is the state vector, containing the state variables, and $\chi$ is a vector of nonlinear functions of $\tau$ and $\mathbf{s}$. The explicit presence of $\tau$ arises from the external dynamic excitation $\mathbf{f}_{\text {ext }}$ (e.g. rotating unbalance). The full nonlinear response for given initial conditions $\mathbf{s}(0)$ can be obtained via transient nonlinear dynamic analysis (TNDA) using readily available advanced integrator routines that were devised for the universal format of eq. (1) [3]. The static equilibrium state $\mathbf{s}=\mathbf{s}_{\mathbf{E}}$ of the system of eq. (1) for a given control parameter can be obtained without integration by setting $\mathbf{s}^{\prime}=\mathbf{0}$ for $\mathbf{f}_{\text {ext }}=\mathbf{0}$ and solving the resulting set of nonlinear algebraic equations. The dynamics of the unforced system for small linear perturbations $\Delta \mathbf{s}$ about $\mathbf{s}=\mathbf{s}_{\mathbf{E}}$ can then be examined by computing the eigenvalues of the Jacobian matrix $\partial \chi /\left.\partial \mathbf{s}\right|_{\mathbf{s}=\mathbf{s}_{\mathbf{E}}}[2,4]$. Included in the resulting information will be the onset of instability of the static equilibrium condition (birth of selfexcited vibration, also known as Hopf bifurcation [2,4]) as the control parameter is changed e.g. the onset 
of instability speed (OIS) in rotordynamics. The complete process of finding $\mathbf{S}_{\mathbf{E}}$ and analysing the linearised dynamics based on the Jacobian is referred to in this paper as static equilibrium and stability analysis (SESA), as in the process introduced by Bonello and Pham for FAB-rotor systems in [5, 6].

In the case of rotordynamic systems with incompressible fluid journal bearings, the instantaneous hydrodynamic pressure, and therefore the journal forces $\mathbf{f}_{\mathbf{J}}=\left[\begin{array}{ll}F_{x} & F_{y}\end{array}\right]^{\mathrm{T}}$, are nonlinear functions of the instantaneous displacements of the journal relative to the housing $\boldsymbol{\varepsilon}=\left[\begin{array}{ll}\varepsilon_{x} & \varepsilon_{y}\end{array}\right]^{\mathrm{T}}$ and the velocities $\boldsymbol{\varepsilon}^{\prime}$ [7]. Hence, in this case, the state vector $\mathbf{s}=\left[\begin{array}{ll}\mathbf{q}^{\mathrm{T}} & \mathbf{q}^{\prime \mathrm{T}}\end{array}\right]^{\mathrm{T}}$, where $\mathbf{q}$ is the vector of generalised coordinates, and $\chi$ contains only the rotor equations of motion. Since $\boldsymbol{\varepsilon}$ is related to $\mathbf{q}$ by simple transformation, the properties of the Jacobian matrix $\partial \chi /\left.\partial \mathbf{s}\right|_{\mathbf{s}=\mathbf{s}_{\mathbf{E}}}$ are governed by the stiffness and damping coefficients contained in the stiffness and damping matrices $\partial \mathbf{f}_{\mathbf{J}} /\left.\partial \boldsymbol{\varepsilon}\right|_{\mathbf{s}=\mathbf{s}_{\mathbf{E}}}, \partial \mathbf{f}_{\mathbf{J}} /\left.\partial \boldsymbol{\varepsilon}^{\prime}\right|_{\mathbf{s}=\mathbf{s}_{\mathbf{E}}} \cdot$ Hence, for incompressible fluid bearings, eigenvalue analysis based on the traditional approach of eight speed-dependent stiffness and damping force coefficients (FC) per bearing, applied to a system of second order differential equations [7], is fully consistent with the linearised dynamical system approach (i.e. the eigenvalue analysis of the state Jacobian $\partial \chi /\left.\partial \mathbf{s}\right|_{\mathbf{s}=\mathbf{s}_{\mathbf{E}}}$ ).

FAB-rotor systems are governed by the coupling of the (compressible) air film, foil structure, and rotor domains, with each domain represented by time-based differential equations within $\chi$ (eq. (1)) [1]. It has been the practice for many years to decouple the air film, foil and rotor equations by approximating the air film equations as algebraic, rather than differential, equations e.g. [8, 9]. The traditional solution technique for the full nonlinear response was therefore a non-simultaneous approach wherein TNDA was only applied to the rotor equations, inevitably involving "time lagging" between the air film equations and those of the foil and rotor $[1,5,6,10,11]$. Such an approach does not preserve the true simultaneous coupling between the three domains [5] since it is not based on the dynamical system representation of eq. (1) but nonetheless it remains popular e.g. [12, 9].

A rotordynamic system with FABs was first expressed as a dynamical system (eq. (1)) in 2013/14 by Bonello and Pham $[13,5,6]$, who then performed the simultaneous solution (i.e. applied TNDA to the 
complete system), and the approach has been taken up by other researchers e.g. $[10,11,14,15,16]$. In this case, the state vector $\mathbf{s}$ contains state variables from all three domains (air film, foil and rotor) e.g. in the case of a symmetric rotor and a foil modelled using the simple equivalent foundation model (SEFM $[11]), \mathbf{s}=\left[\begin{array}{llll}\boldsymbol{\Psi}^{\mathrm{T}} & \widetilde{\mathbf{w}}^{\mathrm{T}} & \mathbf{q}^{\mathrm{T}} & \mathbf{q}^{\prime \mathrm{T}}\end{array}\right]^{\mathrm{T}}[5,6]$ where $\widetilde{\mathbf{w}}$ is the vector of non-dimensional foil deflections at discrete points and $\boldsymbol{\Psi}$ is the vector of instantaneous values of the air film variable $\psi \equiv \tilde{p} \tilde{h}$ used in the Reynolds Equation (RE), discretised at points on a mesh, where $\tilde{p}, \tilde{h}$ are the non-dimensional pressure and film thickness respectively. The journal bearing forces $\mathbf{f}_{\mathbf{J}}$ (obtained by spatial integration of $\tilde{p}$ ) are functions of $\boldsymbol{\psi}, \widetilde{\mathbf{w}}$ and the journal displacements in $\boldsymbol{\varepsilon}$ [5]. In such a situation there are two compelling reasons that motivate the avoidance of the FC method for analysing the free vibration response in the neighbourhood of $\mathbf{s}=\mathbf{s}_{\mathbf{E}}$, particularly the computation of vibration modes (Campbell diagram analysis):

- The FC-method eliminates the air film and foil state variables $\boldsymbol{\Psi}, \widetilde{\mathbf{w}}$ from the analysis through an assumed relationship between the pressure and the journal motion (see below).

- The above assumption has not been tested by verifying individual FC-computed vibration modes by (low amplitude) TNDA of the original system (eq. (1)) - indeed, significant discrepancies in the predictions for the stability of the leading mode (which governs the OIS) have been reported [14].

On the other hand, a linearised dynamical system approach based on the state Jacobian $\partial \chi /\left.\partial \mathbf{s}\right|_{\mathbf{s}=\mathbf{s}_{\mathbf{E}}}$, has been shown to be invariably consistent with the free TNDA response in the neighbourhood of $\mathbf{s}=\mathbf{s}_{\mathbf{E}}[1$, $5,6]$.

The FC method, as used in FAB-rotor systems, is based on Lund's method [17], who used the procedure on gas bearings with rigid sleeves (no foil) and oil-lubricated bearings [14]. According to [14], one of the first contributions that applied Lund's method to FABs was [18] and it is used extensively [8, 9, 19-25] to predict stability of FAB-rotor systems, as well as other features of their linear dynamics e.g. synchronous unbalance response. For gas bearings (including FABs), the air-film pressure $p$ (and hence the bearing force) is not a normal function of the instantaneous journal displacements $\boldsymbol{\varepsilon}=\left[\begin{array}{ll}\varepsilon_{x} & \varepsilon_{y}\end{array}\right]^{\mathrm{T}}$ and velocities $\boldsymbol{\varepsilon}^{\prime}$ since $p$ is actually related to $\boldsymbol{\varepsilon}(\tau)$ through a time-based differential equation arising from the presence of 
$\partial \psi / \partial \tau$ (or $\partial \tilde{p} / \partial \tau$ ) in the compressible RE [26] (which is why $\boldsymbol{\psi}$ is a component of $\mathbf{s}$ in eq. (1)). Hence, the stiffness and damping coefficients cannot be found directly by differentiation. Nonetheless, Lund's FC method assumes that $p$ is a normal function of $\boldsymbol{\varepsilon}, \boldsymbol{\varepsilon}^{\prime}$, which are assumed to be harmonic of assumed frequency (whirl frequency). $p$ is then approximated by a first-order Taylor series expansion [17, 18], which is substituted into the RE (considering $\partial \tilde{p} / \partial \tau$ ) and the foil equations $[18,14]$. The resulting equations are solved for the static equilibrium condition (nonlinear equation with zeroth order term of Taylor expansion as the unknown) and the perturbation part (linear equation with first order terms as unknowns). The latter are integrated to yield the FC (stiffness and damping matrices) of the FAB, which are functions of both the rotational speed $\Omega$ and the assumed whirl frequency $[18,14]$.

Significant discrepancies have been reported by Larsen et al. [14] between predictions for stability determined by the eigenvalue problem based on Lund's FC approach and those determined by TNDA of the free dynamical system (eq. (1)) at low amplitudes. Kim [8] also reported significant discrepancies but the applied TNDA followed the non-simultaneous approach described earlier. The theoretical study by Larsen et al [14] focused on a three-pad FAB test rig model that was previously experimentally validated in [11]. Considering three different values for bump foil stiffness, it was found in [14] that the discrepancy in OIS increased from $5.83 \%$ when the foil was practically rigid, to $28.1 \%$ at the design value of the stiffness, and up to $37.9 \%$ when the design stiffness was halved (Table 1). The error in the whirl frequency was less severe. Larsen et al [14] postulated that the fundamental flaw with Lund's method was that it was originally intended for rigid sleeve gas bearings and its extension to FABs should therefore allow for the influence of the foil deflection when performing the Taylor series approximation of the pressure $p$ [14]. However, the researchers in [14] could not account with certainty for the $5.83 \%$ discrepancy when the foil was rigid. A possible reason suggested in [14] was that the Gümbel condition (see section 2.3.3) was applied once in the TNDA and twice in Lund's method.

The SESA method introduced in $[5,6]$ is the present author's linear alternative to Lund's FC method. As will be shown in the present paper, instead of finding the OIS using the TNDA approach of [14], the same OIS and the associated whirl frequency could be precisely determined in a less time-consuming way 
using the SESA method of finding the leading eigenvalue of the state Jacobian. Furthermore, the result for OIS will always precisely match that by TNDA, regardless of the use of the Gümbel condition.

Table 1. Discrepancies between Lund's force coefficient stability analysis and transient nonlinear dynamic analysis (TNDA) of dynamical system (eq. (1)) for predictions for onset of instability speed (OIS), as found by Larsen et al [14].

\begin{tabular}{|c|c|c|c|c|c|c|}
\hline $\begin{array}{c}\text { bump foil } \\
\text { stiffness } \\
\left(\mathbf{N m}^{-\mathbf{1}} / \mathbf{m}^{\mathbf{2}}\right)\end{array}$ & $\begin{array}{c}\text { OIS - Lund } \\
(\mathbf{r p m})\end{array}$ & $\begin{array}{c}\text { OIS - TNDA } \\
(\mathbf{r p m})\end{array}$ & $\begin{array}{c}\% \\
\text { difference } \\
\text { in OIS }\end{array}$ & $\begin{array}{c}\text { unstable } \\
\text { whirl frequency } \\
\text { at OIS - Lund } \\
\mathbf{( H z})\end{array}$ & $\begin{array}{c}\text { unstable whirl } \\
\text { frequency } \\
\text { at OIS - TNDA } \\
\mathbf{( H z )}\end{array}$ & $\begin{array}{c}\text { \% difference in } \\
\text { unstable whirl } \\
\text { frequency } \\
\text { at OIS }\end{array}$ \\
\hline $8.8 \times 10^{16}$ & 20,583 & 19,450 & 5.83 & 95.2 & 95.9 & -0.73 \\
\hline $8.8 \times 10^{9}$ & 37,039 & 28,900 & 28.1 & 99.8 & 95.9 & 4.07 \\
\hline $4.4 \times 10^{9}$ & 43,287 & 31,400 & 37.9 & 93.9 & 90.4 & 3.87 \\
\hline
\end{tabular}

Campbell diagrams, first introduced in [27], describe the variation with rotational speed of the frequencies of the modes of small amplitude (linear) free vibration of the rotor about the static equilibrium condition (whirl modes). The eigenvalue analysis to produce Campbell diagrams produces essential information associated with each point: damping factor (hence, the stability), mode shape, whirl direction. Hence, Campbell diagram analysis can be regarded as an expansion of the stability analysis (which focuses only on the mode with lowest damping i.e. the leading mode). For this reason, Campbell diagrams are an important tool that aids the interpretation of the dynamic response of the full nonlinear system [19, 22-24, 28]. However, Campbell diagrams for FAB-rotor systems are currently based on Lund's FC method [19, 22-24, 28]. In the light of the above, it is important to establish a method for deriving Campbell diagrams of FAB-rotor systems that completely bypasses Lund's FC method.

The novel contribution of this paper is the generation, for the first time, of the Campbell diagrams of a generic FAB-rotor system via direct extraction from its dynamical system model. This new methodology produces Campbell diagrams that are always completely consistent with TNDA (at low amplitudes) of the dynamical system, and can be verified as such for all modes. This verification is done using "modespecific" initial conditions for TNDA, where initial conditions $\mathbf{s}(0)$ are derived from the Campbell 
diagram analysis in order to make the free dynamical system in eq. (1) respond only in any chosen mode of the Campbell diagram in the neighbourhood of the static equilibrium condition. The new Campbell diagram analysis is based on an eigenvalue analysis of the state Jacobian. As will be revealed in section 3.2 , the number of eigenmodes of the Jacobian at a given speed far exceeds the number of degrees of freedom of the rotordynamic system (due to the air film and foil state variables), and so the algorithm needs to be able to filter these to retain only the rotor whirl modes.

\section{Figure 1}

The novel contribution of this paper constitutes the latest addition to the generic rotor-FAB computational suite developed at the University of Manchester (Figure 1, with novel part highlighted). The ongoing development of the full suite since 2013 has been tracked in various publications $[13,5,6,1,29]$. All its components derive from the universal dynamical system representation of eq. (1), ensuring consistency between their individual results.

Table 2. Case studies considered in this paper (DOF: degrees of freedom; SEFM: simple equivalent foundation model; FFSMM: full foil structure modal model; FLE/CTE: free leading edge / clamped trailing edge).

\begin{tabular}{|c|c|c|c|c|c|}
\hline case & $\begin{array}{c}\text { rotor type } \\
\text { (DOF) }\end{array}$ & $\begin{array}{c}\text { bearing type } \\
\text { (Figure 2) }\end{array}$ & $\begin{array}{c}\text { foil model } \\
\text { (sec. 2.2) }\end{array}$ & $\begin{array}{c}\text { air film boundary conditions (sec. } \\
\text { 2.3.3) }\end{array}$ & references \\
\hline $\begin{array}{c}1 \\
(\mathrm{sec} .4)\end{array}$ & rigid, 4 DOF & 3-pad & SEFM & finite $\theta$, Gümbel & {$[14,11,10]$} \\
\hline $\begin{array}{c}2 \\
(\mathrm{sec} .5)\end{array}$ & rigid, 2 DOF & 1-pad & FFSMM & cont. $\theta$, no truncation (FLE/CTE) & {$[29]$} \\
\hline $\begin{array}{c}4 \\
(\mathrm{sec} .6)\end{array}$ & flexible & 1-pad & SEFM & cont. $\theta$, no truncation (FLE/CTE) & {$[30]$} \\
\hline
\end{tabular}

\section{Figure 2}


The new methodology is tested on the three case studies presented in Table 2, all of which refer to systems from the literature that have been expressed in dynamical system form. The focus of the validation will be Case no.1 (see Table 2), which was not previously analysed by the author, and for which the results in Table 1 were obtained [14]. It relates to a Siemens test rig with 3-pad FABs (Figure 2(a)) that was previously studied by Larsen and Santos $[14,11,10]$, who used the Bubnov-Galerkin Finite Element (FE) to model the air film. Since the present author uses Finite Difference (FD) to model the air film, the FD model of the 3-pad bearing is derived from first principles (section 2.3) and integrated into the authors' component mode synthesis computational suite (Figure 1). As a preliminary check, TNDA results from the rotor-FAB model are correlated against those published in [10] (section 4.1). The full SESA based on the parameters in [14] is then performed in section 4.2, where the results, including novel information not generated in $[14,11]$ (i.e. Campbell diagrams, mode shapes,...etc), are validated against TNDA and also correlated with observations in $[14,11]$.

Cases nos. 2, 3 in Table 2 cover systems with single-pad FAB (Figure 2(b)) previously studied by the present author. Case no.2 is included to illustrate applicability of the novel method to foil models that are more complex than the SEFM (in this case the full foil structure modal model (FFSMM), which accounts elastic interaction between the bumps and the inertia of the bump foil [29]). Finally, Case no.3 is included to illustrate the output of the method for a flexible rotor supported on two FABs.

The generalised theory covering all three cases is presented in section 2 (Modelling) and section 3 (Computation and Analysis). The results are presented and discussed in sections 4-6.

As an introduction to the original theory of this paper, the reader may refer to a contemporaneous conference paper by the author [31], wherein the Campbell diagram analysis is developed specifically for the simplest case of rotor-FAB system (a rigid symmetric rotor on single-pad FABs with pad modelled by SEFM, which is not considered in Table 2).

\section{MODELLING}


The differential equations governing the nonlinear dynamics of the generic rotor-FAB system in Figure 3 can be expressed in the dynamical system form of eq. (1) as follows:

$$
\begin{aligned}
& \boldsymbol{\Psi}_{\mathrm{L}}^{\prime}=\mathbf{g}_{\mathrm{RE}_{\mathrm{L}}}\left(\boldsymbol{\Psi}_{\mathrm{L}}, \widetilde{\mathbf{w}}_{\mathrm{L}}\left(\mathbf{s}_{\mathrm{F}_{\mathrm{L}}}\right), \boldsymbol{\varepsilon}_{\mathrm{L}}(\mathbf{q})\right), \mathbf{s}_{\mathrm{F}_{\mathrm{L}}}{ }^{\prime}=\mathbf{g}_{\mathrm{Foil}_{\mathrm{L}}}\left(\boldsymbol{\Psi}_{\mathrm{L}}, \mathbf{s}_{\mathrm{F}_{\mathrm{L}}}, \boldsymbol{\varepsilon}_{\mathrm{L}}(\mathbf{q})\right) \\
& \boldsymbol{\Psi}_{\mathrm{R}}{ }^{\prime}=\mathbf{g}_{\mathrm{RE}}\left(\boldsymbol{\Psi}_{\mathrm{R}}, \widetilde{\mathbf{w}}_{\mathrm{R}}\left(\mathbf{s}_{\mathrm{F}_{\mathrm{R}}}\right), \boldsymbol{\varepsilon}_{\mathrm{R}}(\mathbf{q})\right), \mathbf{s}_{\mathrm{F}_{\mathrm{R}}}{ }^{\prime}=\mathbf{g}_{\mathrm{Foil}_{\mathrm{R}}}\left(\boldsymbol{\Psi}_{\mathrm{R}}, \mathbf{s}_{\mathrm{F}_{\mathrm{R}}}, \boldsymbol{\varepsilon}_{\mathrm{R}}(\mathbf{q})\right)
\end{aligned}
$$

where: the non-dimensional time is defined as $\tau=\Omega t / 2$ ( $\Omega$ being the rotational speed); the subscripts "L", "R" used throughout refer to the left and right hand bearings in Figure 3; and the vector state variable $\mathbf{s}$ comprises sub-vectors containing state variables relating to the air films $\left(\boldsymbol{\Psi}_{\mathrm{L}}, \boldsymbol{\Psi}_{\mathrm{R}}\right)$, the respective foils $\left(\mathbf{s}_{\mathrm{F}_{\mathrm{L}}}, \mathbf{s}_{\mathrm{F}_{\mathrm{R}}}\right)$ and the rotor $\left(\mathbf{q}, \mathbf{q}^{\prime}\right)$ :

$$
\mathbf{s}=\left[\begin{array}{llllll}
\boldsymbol{\Psi}_{\mathrm{L}}^{\mathrm{T}} & \mathbf{s}_{\mathrm{F}_{\mathrm{L}}}^{\mathrm{T}} & \boldsymbol{\Psi}_{\mathrm{R}}^{\mathrm{T}} & \mathbf{s}_{\mathrm{F}_{\mathrm{R}}}^{\mathrm{T}} & \mathbf{q}^{\mathrm{T}} & \mathbf{q}^{\prime T}
\end{array}\right]^{\mathrm{T}}
$$

The details of these three equation sets $(2 \mathrm{a}, \mathrm{c}),(2 \mathrm{~b}, \mathrm{~d}),(2 \mathrm{e})$, pertaining to the air film, foil structure and rotor domains respectively, are described in turn in the following three sub-sections, starting with eqs. (2e).

\section{Figure 3}

\subsection{Rotor Equations (eqs. (2e))}

As in the author's previous FAB work [1], the Adams [32] approach for rotor systems with generic nonlinear bearings is used. The physical degrees of freedom of the rotor are transformed to modal coordinates using a truncated series of component modes. These are the undamped free vibration modes of the linear part at zero rotational speed, where the linear part is the given system minus the nonlinear 
elements (FABs) (i.e. FABs replaced by gaps). The modal transformation uses $H$ modes and $\boldsymbol{\Lambda}$ is the $H \times H$ diagonal matrix of the squares of their circular frequencies. The Cartesian coordinates of any point $\mathrm{P}$ along the rotor are therefore given by:

$$
\mathbf{v}_{\mathbf{P}}=\left[\begin{array}{ll}
x_{\mathrm{P}} & y_{\mathrm{P}}
\end{array}\right]^{\mathrm{T}}=\mathbf{H}_{\mathbf{v}_{\mathbf{P}}} \mathbf{q}(\tau)=\left[\begin{array}{lll}
\boldsymbol{\varphi}_{\mathbf{P}}^{(1)} & \cdots & \boldsymbol{\varphi}_{\mathbf{P}}^{(H)}
\end{array}\right] \mathbf{q}(\tau)
$$

where $\mathbf{q}$ is the $H \times 1$ column matrix (vector) of modal coordinates and the $H$ columns of the modal matrix $\mathbf{H}_{\mathbf{v}_{\mathbf{P}}}$ are the mass-normalised eigenvectors of the respective modes containing the modal displacements evaluated at the degrees of freedom in $\mathbf{v}_{\mathbf{P}}=\left[\begin{array}{ll}x_{\mathrm{P}} & y_{\mathrm{P}}\end{array}\right]^{\mathrm{T}}$.

The term $\mathbf{H}_{\mathbf{g}}^{\mathrm{T}} \mathbf{P} \mathbf{H}_{\boldsymbol{\alpha}} \dot{\mathbf{q}}$, defined in [33], accounts for the gyroscopic effect which is assumed to be concentrated at a number of locations distributed along the rotor. $\mathbf{f}_{\mathrm{J}_{\mathrm{L}, \mathrm{R}}}$ are the $2 \times 1$ vectors of air film pressure forces exerted by the $\mathrm{L}, \mathrm{R}$ FABs on the respective journals in the $x, y$ directions. $\mathbf{f}_{\mathbf{u}}$ the $2 n_{\mathrm{U}} \times 1$ vector of rotational unbalance forces, alternately in the $x, y$ directions, distributed over $n_{\mathrm{U}}$ planes, and $\mathbf{f}_{\mathbf{s}}$

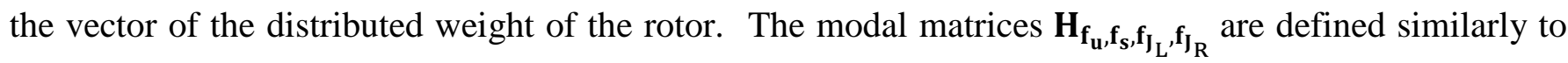
$\mathbf{H}_{\mathbf{v}_{\mathbf{P}}}$ with respect to the corresponding excitation force vectors.

Taking the left hand FAB as an example, the forces in $\mathbf{f}_{\mathrm{J}_{\mathrm{L}}}$ are obtained by integrating the air film pressure distribution $p(\xi, \theta, \tau)$ over the bearing area:

$$
\mathbf{f}_{\mathbf{J}_{\mathrm{L}}}=-2 R^{2} \int_{\xi=0}^{0.5 L / R} \int_{\theta=0}^{2 \pi}\left(p(\xi, \theta, \tau)-p_{\mathrm{a}}\right)\left[\begin{array}{c}
\cos \theta \\
\sin \theta
\end{array}\right] d \theta d \xi
$$

where $R$ and $L$ are the radius and length respectively of the $\mathrm{FAB}, \xi=\frac{z_{\text {local }}}{R}$ the non-dimensional local axial coordinate and $p_{\mathrm{a}}$ is the absolute atmospheric pressure. As seen from eq. (2e), $\mathbf{f}_{\mathbf{J}_{\mathrm{L}}}$ can be calculated for known air film state variables in $\boldsymbol{\psi}_{\mathrm{L}}$, known foil state variables $\mathbf{s}_{\mathrm{F}_{\mathrm{L}}}$, and known journal eccentricity vector $\varepsilon_{\mathrm{L}}$, which, for a rigid support structure, is given by the absolute journal coordinates $x_{\mathrm{J}_{\mathrm{L}}}, y_{\mathrm{J}_{\mathrm{L}}}$ as follows (where $c_{\mathrm{L}}$ is the nominal radial clearance of the left FAB):

$$
\boldsymbol{\varepsilon}_{\mathrm{L}}=\left[\begin{array}{ll}
x_{\mathrm{J}_{\mathrm{L}}} / c_{\mathrm{L}} & y_{\mathrm{J}_{\mathrm{L}}} / c_{\mathrm{L}}
\end{array}\right]=\mathbf{H}_{\mathrm{f}_{\mathrm{J}_{\mathrm{L}}}} \mathbf{q}(\tau) / c_{\mathrm{L}}
$$




\subsection{Foil Equations (eqs. (2b,d))}

In the case studies of the present work (Table 2), two foil models are used:

(a) the simple elastic foundation model (SEFM) $[14,11,5,6]$;

(b) the full foil structure modal model (FFSMM) [29, 1].

Both models have been successfully used in experimentally validated studies e.g. [11, 6] (SEFM) and [1, 29] (FFSMM). Both models are based entirely on the bump foil i.e. the stiffness and inertia of the top foil are neglected and it is assumed to be in contact with the bumps at all times for the purpose of determining the local radial deflection $w$ of the top foil, which is assumed to be a function of $\theta$ only. Defining $\widetilde{w}=w / c$, and taking the left hand FAB as an example, the vector $\widetilde{\mathbf{w}}_{\mathrm{L}}$ of non-dimensional deflections at discrete values $\theta_{j}$ of $\theta$ defining the clearance boundary, is determined from the state variables of the foil model:

$$
\widetilde{\mathbf{w}}_{\mathrm{L}}(\tau)=\mathbf{T}_{\mathrm{F}_{\mathrm{L}}} \mathbf{s}_{\mathrm{F}_{\mathrm{L}}}(\tau)
$$

where $\mathbf{T}_{\mathrm{F}_{\mathrm{L}}}$ is a constant transformation matrix dependent on the foil model used.

For the SEFM, $\mathbf{T}_{\mathrm{F}_{\mathrm{L}}}$ is an identity matrix and the vector function $\mathbf{g}_{\mathrm{Foil}_{\mathrm{L}}}$ in eq. (2b) is given by:

$$
\mathbf{g}_{\mathrm{Foil}_{\mathrm{L}}} \equiv \frac{2}{\eta_{\mathrm{L}}}\left(\frac{\mathbf{p}_{\mathrm{g}, \theta_{\mathrm{L}}}\left(\boldsymbol{\Psi}_{\mathrm{L}}, \widetilde{\mathbf{w}}_{\mathrm{L}}\left(\mathbf{s}_{\mathrm{F}_{\mathrm{L}}}\right), \boldsymbol{\varepsilon}_{\mathrm{L}}(\mathbf{q})\right)}{c_{\mathrm{L}} K_{\mathrm{b}_{\mathrm{L}}}}-\mathbf{s}_{\mathrm{F}_{\mathrm{L}}}\right), \quad\left(\mathbf{s}_{\mathrm{F}_{\mathrm{L}}} \equiv \widetilde{\mathbf{w}}_{\mathrm{L}}\right)
$$

where $K_{\mathrm{b}_{\mathrm{L}}}$ is the stiffness per unit area of the bump foil and $\eta_{\mathrm{L}}$ is damping loss factor. $\mathbf{p}_{\mathrm{g}, \theta_{\mathrm{L}}}$ is the vector of the averages of the gauge pressure $p_{\mathrm{g}}\left(=p-p_{\mathrm{a}}\right)$ over the $\xi$-direction for discrete values of $\theta, \theta_{j}$ :

$$
\mathbf{p}_{\mathrm{g}, \theta_{\mathrm{L}}}=\left[\begin{array}{lll}
\cdots & p_{\mathrm{g}, \theta}\left(\theta_{j}\right) & \cdots
\end{array}\right]^{\mathrm{T}} \quad p_{\mathrm{g}, \theta}\left(\theta_{j}\right)=\frac{2 R}{L} \int_{\xi=0}^{0.5 L / R}\left(p\left(\xi, \theta_{j}, \tau\right)-p_{\mathrm{a}}\right) d \xi
$$


Eq. (8a) assumes an equivalent viscous damping coefficient of $C_{\text {damp,eq }}=K_{\mathrm{b}_{\mathrm{L}}} \eta_{\mathrm{L}} / \Omega[5,6,11,14]$. The use of $\Omega$ rather than the actual vibration frequency $\omega$ for $C_{\text {dampeq }}$ is necessary to work in the time domain where $\omega$ is unknown and the frequency spectrum will generally contain more than one frequency. However, the validity of this assumption (also used in $[8,9]$ ) has been justified by theoretical studies in [5] using the harmonic balance method, as well as experimentally validated studies e.g. [11].

The FFSMM is based on the principle that the vibrating shape of the bump foil can be represented by a truncated series of $n_{\mathrm{F}}$ undamped modes (bump foil component modes). The vector function $\mathbf{g}_{\mathrm{Foil}}$ in eq. (2b) is therefore given by:

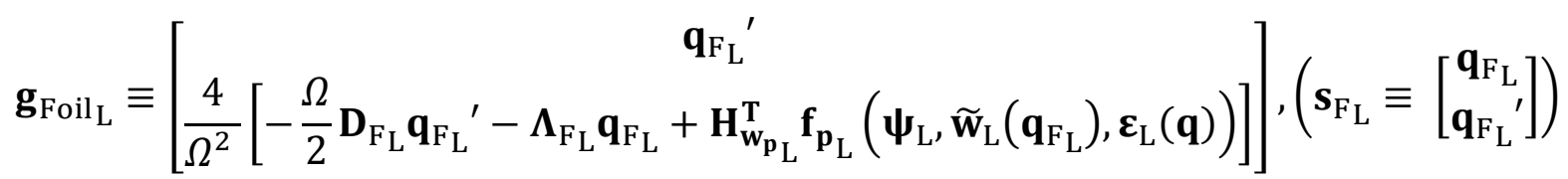

where $\mathbf{q}_{\mathrm{F}_{\mathrm{L}}}$ is the vector of modal coordinates of the foil, $\boldsymbol{\Lambda}_{\mathrm{F}_{\mathrm{L}}}$ is the diagonal matrix of the squares of the natural circular frequencies of the foil modes, and $\mathbf{D}_{\mathrm{F}_{\mathrm{L}}}$ is the modal damping matrix. $\mathbf{H}_{\mathbf{w}_{\mathbf{p}_{\mathrm{L}}}}$ is the matrix whose $n_{\mathrm{F}}$ columns are the $n_{\text {bumps }} \times 1$ mass-normalised eigenvectors containing the radial displacements at the apexes of the bumps. $\mathbf{f}_{\mathbf{p}_{\mathrm{L}}}$ is the $n_{\text {bumps }} \times 1$ vector of air pressure forces on the individual bumps and each element of $\mathbf{f}_{\mathbf{p}_{\mathrm{L}}}$ can be determined by integrating the regional air film pressure distribution over a bump projected area of $S \times L$, where $S$ is the pitch [1]:

$$
\mathbf{f}_{\mathbf{p}_{\mathrm{L}}}=\left[\begin{array}{lll}
\cdots & f_{n} & \cdots
\end{array}\right]^{\mathrm{T}}, \quad f_{n}=-2 R^{2} \int_{\xi=0}^{0.5 L / R} \int_{\theta=\theta_{k}-S / R}^{\theta_{k}+S / R}\left(p(\xi, \theta, \tau)-p_{\mathrm{a}}\right) d \theta d \xi
$$

In the FFSMM, the foil deflections $\widetilde{\mathbf{w}}_{\mathrm{L}}$ in eq. (7) are obtained by interpolation from the radial displacements at the apexes of the bumps contained in vector $\mathbf{w}_{\mathbf{p}_{\mathrm{L}}}$. Hence, in eq. (7), $\mathbf{T}_{\mathrm{F}_{\mathrm{L}}}=\mathbf{T}_{2} \mathbf{T}_{1}$, where $\mathbf{T}_{1}$ is a matrix that transforms from $\mathbf{s}_{\mathrm{F}_{\mathrm{L}}}$ (eq. (10b)) to $\mathbf{w}_{\mathbf{p}_{\mathrm{L}}}$ (see [29]), and $\mathbf{T}_{2}$ is a matrix of interpolating coefficients that transforms from $\mathbf{w}_{\mathbf{p}_{\mathrm{L}}}$ to $\widetilde{\mathbf{w}}_{\mathrm{L}}$. 


\subsection{Air Film Equations (eqs. (2a,c))}

Eqs. (2a,c) each comprise the discretised form of the isothermal RE of the each corresponding air film. Temporarily dropping the subscripts $\mathrm{L}$ and $\mathrm{R}$, the $\mathrm{RE}$ governs the pressure function $p(\xi, \theta, \tau)$ and is formulated in terms of the combined state variable $\psi \equiv \tilde{p} \tilde{h}$ where $\tilde{p}=\frac{p}{p_{\mathrm{a}}}$ and $\tilde{h}=\frac{h}{c}$ are the nondimensional air film pressure and thickness at a position $(\xi, \theta)(c$ being the nominal radial clearance and is replaced by $c_{\mathrm{L}}$ or $c_{\mathrm{R}}$ according to the bearing considered):

$$
\psi^{\prime}=\frac{1}{B}\left\{\frac{\partial}{\partial \theta}\left[\psi\left(\tilde{h} \frac{\partial \psi}{\partial \theta}-\psi \frac{\partial \widetilde{h}}{\partial \theta}\right)\right]+\frac{\partial}{\partial \xi}\left[\psi\left(\tilde{h} \frac{\partial \psi}{\partial \xi}-\psi \frac{\partial \widetilde{h}}{\partial \xi}\right)\right]\right\}-\frac{\partial \psi}{\partial \theta}
$$

$B=\frac{6 \mu \Omega}{p_{\mathrm{a}}}\left(\frac{R}{c}\right)^{2}$ is the bearing number ( $\mu$ is the air viscosity).

\subsubsection{Three-pad bearing}

With reference to Figure 2(a), each pad no. $k$ has a total angular extent of $\alpha^{(k)}$ and an initial slope wherein the profile rises gradually by $h_{\mathrm{s}}^{(k)}\left(=c \tilde{h}_{\mathrm{s}}^{(k)}\right)$ over an angular extent of $\beta^{(k)}$. The RE of eq. (12) is applied to each pad no. $k$, for which the domain of $\theta$ is denoted by $\theta^{(k)}$, starting at $\theta_{\mathrm{st}}^{(k)}$ i.e.

$$
\theta^{(k)} \in\left(\theta_{\mathrm{st}}^{(k)} \leq \theta \leq \theta_{\mathrm{st}}^{(k)}+\alpha^{(k)}\right)
$$

The non-dimensional film thickness is therefore given by:

$$
\tilde{h}\left(\theta^{(k)}\right)=1-\boldsymbol{\varepsilon}^{\mathrm{T}}\left[\begin{array}{c}
\cos \theta^{(k)} \\
\sin \theta^{(k)}
\end{array}\right]+\widetilde{w}\left(\theta^{(k)}\right)+\tilde{h}_{\mathrm{add}}\left(\theta^{(k)}\right)
$$

where

$$
\tilde{h}_{\mathrm{add}}\left(\theta^{(k)}\right)=\left\{\begin{array}{cl}
\tilde{h}_{\mathrm{s}}^{(k)}\left(\theta_{\mathrm{st}}^{(k)}+\beta^{(k)}-\theta^{(k)}\right) / \beta^{(k)} & , \theta^{(k)} \leq \theta_{\mathrm{st}}^{(k)}+\beta^{(k)} \\
0 & , \theta^{(k)}>\theta_{\mathrm{st}}^{(k)}+\beta^{(k)}
\end{array}\right.
$$


The RE for each pad is discretised over a finite difference (FD) grid. The rectangular grid has $N_{z} \times N_{\theta}$ points spaced by $\Delta \xi, \Delta \theta$ in the respective directions where $\xi=\xi_{i}, i=1, \ldots, N_{z}$ and $\theta^{(k)}=\theta_{j}^{(k)}, j=$ $1, \ldots, N_{\theta}$. In the $\xi$ direction the grid extends from $\xi_{1}=\Delta \xi$ to $\xi_{N_{z}}=0.5 L / R$ i.e. covers only half the axial length due to symmetry. In the $\theta^{(k)}$ direction the grid extends from $\theta_{1}^{(k)}=\theta_{\mathrm{st}}^{(k)}+\Delta \theta$ to $\theta_{N_{\theta}}^{(k)}=\theta_{\mathrm{st}}^{(k)}+$ $\alpha^{(k)}-\Delta \theta$. The edges of the pad are excluded from the grid since they are at atmospheric pressure.

Defining $\psi_{i, j}^{(k)}(\tau)=\psi\left(\xi_{i}, \theta_{j}^{(k)}, \tau\right)$ the partial derivatives in eq. (12) are approximated using centraldifference formulae [1]:

$$
\begin{aligned}
& \left.\frac{\partial \psi}{\partial \theta}\right|_{i, j} ^{(k)}=\frac{1}{2 \Delta \theta}\left(\psi_{i, j+1}^{(k)}-\psi_{i, j-1}^{(k)}\right),\left.\frac{\partial^{2} \psi}{\partial \theta^{2}}\right|_{i, j} ^{(k)}=\frac{1}{\Delta \theta^{2}}\left(\psi_{i, j+1}^{(k)}-2 \psi_{i, j}^{(k)}+\psi_{i, j-1}^{(k)}\right) \\
& \left.\frac{\partial \psi}{\partial \xi}\right|_{i, j} ^{(k)}=\frac{1}{2 \Delta \xi}\left(\psi_{i+1, j}^{(k)}-\psi_{i-1, j}^{(k)}\right),\left.\frac{\partial^{2} \psi}{\partial \xi^{2}}\right|_{i, j} ^{(k)}=\frac{1}{\Delta \xi^{2}}\left(\psi_{i+1, j}^{(k)}-2 \psi_{i, j}^{(k)}+\psi_{i-1, j}^{(k)}\right)
\end{aligned}
$$

where the following boundary conditions are applied when calculating the derivatives at the edges of the grid:

$$
\begin{gathered}
\psi_{N_{z}+1, j}^{(k)}=\psi_{N_{z}-1, j}^{(k)}, \psi_{0, j}^{(k)}=\tilde{h}_{j}^{(k)} \\
\psi_{i, N_{\theta}+1}^{(k)}=\tilde{h}\left(\theta^{(k)}=\theta_{\mathrm{st}}^{(k)}+\alpha^{(k)}\right), \psi_{i, 0}^{(k)}=\tilde{h}\left(\theta^{(k)}=\theta_{\mathrm{st}}^{(k)}\right)
\end{gathered}
$$

Eq. (17a) is due to symmetry and eqs. $(17 \mathrm{~b}, \mathrm{c}, \mathrm{d})$ are due to atmospheric conditions. In eq. (17b) $\tilde{h}_{j}^{(k)}=$ $\tilde{h}\left(\theta_{j}^{(k)}\right)$ and is computed from eq. (14). The values of the derivatives of $\tilde{h}$ at the grid points are:

$$
\left.\frac{\partial \widetilde{h}}{\partial \theta}\right|_{j} ^{(k)}=\boldsymbol{\varepsilon}^{\mathrm{T}}\left[\begin{array}{c}
\sin \theta_{j} \\
-\cos \theta_{j}
\end{array}\right]+\left.\frac{\partial \widetilde{w}}{\partial \theta}\right|_{j} ^{(k)}+\left.\frac{\partial \widetilde{h}_{\mathrm{add}}}{\partial \theta}\right|_{j} ^{(k)},\left.\frac{\partial^{2} \widetilde{h}}{\partial \theta^{2}}\right|_{j} ^{(k)}=\boldsymbol{\varepsilon}^{\mathrm{T}}\left[\begin{array}{c}
\cos \theta_{j} \\
\sin \theta_{j}
\end{array}\right]+\left.\frac{\partial^{2} \widetilde{w}}{\partial \theta^{2}}\right|_{j} ^{(k)}+\left.\frac{\partial^{2} \widetilde{h}_{\mathrm{add}}}{\partial \theta^{2}}\right|_{j} ^{(k)}
$$

In eqs. (18), the partial derivatives are computed similarly to eqs. (16) using grid point values $\widetilde{w}_{j}^{(k)}=$ $\widetilde{w}\left(\theta_{j}^{(k)}\right)$ and $\tilde{h}_{\operatorname{add}_{j}}^{(k)}=\tilde{h}_{\text {add }}\left(\theta_{j}^{(k)}\right) . \quad \tilde{h}_{\operatorname{add}_{j}}^{(k)}$ is computed directly from eq. (15), whereas the $\widetilde{w}_{j}^{(k)}$ 
values are determined from the state variables of the differential equations of the corresponding foil eqs. (2b) or $(2 \mathrm{~d})$. The values of $\widetilde{w}$ at the edges of the pad i.e. $\widetilde{w}\left(\theta^{(k)}=\theta_{\mathrm{st}}^{(k)}+\alpha^{(k)}\right), \widetilde{w}\left(\theta^{(k)}=\theta_{\mathrm{st}}^{(k)}\right)$, which are excluded from the grid but required for eqs. $(17 \mathrm{c}, \mathrm{d})$ and in the calculation of the derivatives at the edges of the grid, are calculated by extrapolation from the grid foil deflections $\widetilde{w}_{j}^{(k)}$.

Focusing on the left hand FAB and substituting eqs. (18), (14), (16) into the RE (eq. (12)) yields the FD RE equation set for pad no. $k$, which comprises $N_{z} N_{\theta}$ first order differential equations with $\tau$ as independent variable and $\boldsymbol{\psi}_{\mathrm{L}}^{(k)}$ as the main state vector:

$$
\boldsymbol{\Psi}_{\mathrm{L}}^{(k) \prime}=\mathbf{g}_{\mathrm{RE}}^{(k)}\left(\boldsymbol{\Psi}_{\mathrm{L}}^{(k)}, \widetilde{\mathbf{w}}_{\mathrm{L}}^{(k)}, \boldsymbol{\varepsilon}_{\mathrm{L}}(\mathbf{q})\right)
$$

where $\boldsymbol{\Psi}_{\mathrm{L}}^{(k)}$ and $\widetilde{\mathbf{w}}_{\mathrm{L}}^{(k)}$ are of dimensions $N_{z} N_{\theta} \times 1$ and $N_{\theta} \times 1$ respectively and defined as follows:

$$
\boldsymbol{\Psi}_{\mathrm{L}}^{(k)}=\left[\begin{array}{lll}
\cdots & \psi_{i, j}^{(k)} & \cdots
\end{array}\right]^{\mathrm{T}}, \widetilde{\mathbf{w}}_{\mathrm{L}}^{(k)}=\left[\begin{array}{lll}
\cdots & \widetilde{w}_{j}^{(k)} & \cdots
\end{array}\right]^{\mathrm{T}}
$$

The right hand side of eq. (2a), which defines the full system of discretised RE equations of the left hand $\mathrm{FAB}$, is then obtained by assembling the state space equations of its individual $N_{\mathrm{p}_{\mathrm{L}}}$ pads (where $N_{\mathrm{p}_{\mathrm{L}}}=3$ for the multi-pad FAB used in this paper):

$$
\mathbf{g}_{\mathrm{RE}_{\mathrm{L}}}=\left[\begin{array}{c}
\vdots \\
\mathbf{g}_{\mathrm{RE}}^{(k)} \mathrm{L} \\
\vdots
\end{array}\right], \boldsymbol{\Psi}_{\mathrm{L}}=\left[\begin{array}{c}
\vdots \\
\boldsymbol{\Psi}_{\mathrm{L}}^{(k)} \\
\vdots
\end{array}\right], \widetilde{\mathbf{w}}_{\mathrm{L}}=\left[\begin{array}{c}
\vdots \\
\widetilde{\mathbf{w}}_{\mathrm{L}}^{(k)} \\
\vdots
\end{array}\right]
$$

\subsubsection{Single pad bearing}

For a single pad bearing shown in Figure 2(b), the pad covers the full extent in the $\theta$ direction i.e. from $\theta=\pi / 2$ to $\theta=5 \pi / 2$. As discussed in [1], in view of the uncertainty in the conditions at location $\theta=\pi / 2$ (or $5 \pi / 2)$, two models are considered:
A. Infinite (continuous) pad in $\theta$ direction;
B. Finite pad in $\theta$ direction. 
In the finite $\theta$ model (B), used by Bonello and Bin Hassan in [1], it is assumed that a narrow opening (slit) is formed at $\theta=\pi / 2$ (or $5 \pi / 2$ ) along the $z$ direction, and the pressure is fixed at atmospheric along this line regardless of the dynamics of the journal. The FAB is then a special case of the multi-pad FAB of the previous section, where the number of pads is $1, \theta_{\mathrm{st}}^{(k)}=\pi / 2$, and $\tilde{h}_{\mathrm{add}}=0$ in eq. (14).

In the continuous $\theta$ model (A), used by Bonello and co-authors in $[1,5,6,13,29,30]$, there is no imposition of atmospheric pressure at $\theta=\pi / 2$ and the pressure there is wholly determined by the dynamics. In this case, eqs. $(17 \mathrm{c}, \mathrm{d})$ are replaced by a periodicity condition in the $\theta$ direction:

$$
\psi_{i, N_{\theta}+1}=\psi_{i, 1}, \psi_{i, 0}=\psi_{i, N_{\theta}}
$$

and the grid extends from $\theta_{1}=\pi / 2$ to $\theta_{N_{\theta}}=5 \pi / 2-\Delta \theta$.

\subsubsection{Air film forces on foil and journal}

In eqs. (5), (9b), (11b), the gauge pressure $p-p_{\mathrm{a}} \equiv p_{\mathrm{a}} \times\left(\frac{\psi}{\widetilde{h}}-1\right)$. As discussed in [1], two alternative conditions are considered for the force calculation:

i. Full pressure condition (no truncation).

ii. Gümbel condition (truncation of pressures below atmospheric condition) i.e. $p$ is replaced by $p_{\text {trunc }}$ where $p_{\text {trunc }}=p_{\mathrm{a}}$ in those regions where $p<p_{\mathrm{a}}$ and $p_{\text {trunc }}=p$ elsewhere.

The Gümbel condition is a retrospective correction for the detachment of the top foil from the bump foil in regions of sub-atmospheric pressure, when using SEFM or FFSMM [1]. This condition is appropriate for FABs with pads that have a clamped leading edge and free trailing edge (CLE/FTE), as in the case of the three-pad bearing (Figure 2(a)) $[14,11,10]$. Single-pad FABs are normally used with a free leading edge and clamped trailing edge (FLE/CTE) (as in Figure 2(b)) [1] for which a theoretical study [34] showed that the Gümbel condition may not be appropriate. This was backed by a theoretical and experimental study [1] which showed that a continuous pad model (A in section 2.3.2) with full (no truncation) pressure condition ((i) above) is a satisfactory alternative. 
It is noted that when applying the Gümbel condition, Larsen et al $[14,11,10]$ truncated the pressure used to evaluate the journal forces (eq. (5)) but did not truncate the pressure used within the foil equations (eqs. (9b)). The truncation of this latter pressure had little effect on the results $[14,11,10]$, as can be confirmed by the author of the present paper, who will therefore follow the same approach.

\section{Computation and Analysis}

This section focuses on the development of the highlighted area of the chart in Figure 1.

\subsection{The Jacobian and its Computation}

The Jacobian matrix of the dynamical system of eq. (1) is defined as:

$$
\frac{\partial \chi}{\partial \mathbf{s}}=\left[\begin{array}{lllll}
\frac{\partial \chi}{\partial s_{1}} & \cdots & \frac{\partial \chi}{\partial s_{n}} & \cdots & \frac{\partial \chi}{\partial s_{N_{s}}}
\end{array}\right], \quad\left(\mathbf{s}=\left[\begin{array}{lllll}
s_{1} & \cdots & s_{n} & \cdots & s_{N_{\mathbf{s}}}
\end{array}\right]^{\mathrm{T}}\right)
$$

This matrix is required by the following processes in the full suite depicted in Figure 1.

1) Transient nonlinear dynamic analysis (TNDA). In this process eqs. (2a-e) are solved for the time history of the nonlinear response, typically at fixed rotational speed and a certain level of unbalance excitation (or none at all). The solver used is an implicit time domain integrator like the function ode23s in Matlab, which is a stiff solver with automatic time-step control for maintaining the numerical accuracy within a prescribed tolerance [3]. The Jacobian matrix is required to be computed at each time step.

2) The first part of the static equilibrium and stability analysis (SESA). This process involves the determination of static equilibrium configuration at each given rotational speed by setting $\mathbf{s}^{\prime}=\mathbf{0}$ in eq. (1) and finding the solution $\mathbf{s}=\mathbf{s}_{\mathrm{E}}$ of the resulting system of nonlinear algebraic equations

$$
\left.\chi(\tau, \mathbf{s})\right|_{\mathbf{f}_{\mathrm{u}}=\mathbf{0}}=\mathbf{0}
$$


The Jacobian is needed here to solve eq. (24) using the damped Newton-Raphson iterative method [35]. At the beginning of the iterative process at each given speed, the Jacobian computed is

$$
\left.\frac{\partial \chi}{\partial s}\right|_{f_{u}=0, s=s_{E}^{(0)}}
$$

where $\mathbf{s}=\mathbf{s}_{\mathbf{E}}^{(0)}$ is the initial approximation for the solution $\mathbf{s}=\mathbf{s}_{\mathrm{E}}$ (obtained from the static equilibrium solution at the previous speed, or from TNDA in the case of the first speed). For subsequent iterations, the required inverse of the Jacobian is updated using the approximation of Broyden's Method [35]. After convergence has been achieved, the Jacobian at $\mathbf{s}=\mathbf{s}_{\mathrm{E}}$ is computed for the second part of SESA.

3) The second part of SESA. This requires the eigenvalue analysis of the state Jacobian matrix for each speed:

$$
J=\left.\frac{\partial \chi}{\partial s}\right|_{f_{u}=0, s=s_{E}}
$$

The data from this computation is then used for stability analysis and the extraction of Campbell diagrams and associated information highlighted in Figure 1.

The Jacobian of the vector function $\chi$ can be calculated in two ways:

- Full numerical approach;

- Analytical/part-analytical approach.

The full numerical approach can be done in Matlab by applying the subroutine numjac.m [3], which evaluates numerically each column of the Jacobian in turn, as per basic definition of eq. (23a). This process therefore involves $N_{\mathbf{s}}+1$ evaluations of $\boldsymbol{\chi}$, making it time consuming and completely unfeasible for the TNDA. Hence, an analytical/part-analytical approach is used to compute the Jacobian, which also accelerates the SESA.

\subsubsection{Analytical/part-analytical Jacobian}


The Jacobian is written as

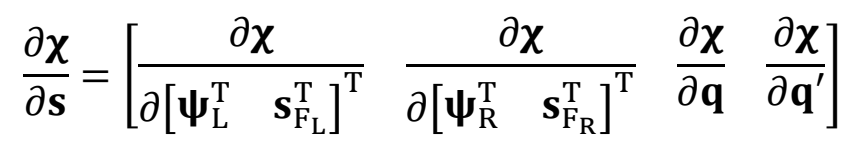

The sub-matrices in eq. (27) are given by the expressions below, where the following notation is adopted: $\mathbf{0}_{m \times n}$ denotes an $m \times n$ matrix of zeros; $\mathbf{I}_{m \times m}$ denotes an $m$-square identity matrix; $N_{\mathbf{s}_{\mathrm{F}_{\mathrm{L}}}}, N_{\mathbf{S}_{\mathrm{F}_{\mathrm{R}}}}$ denote the number of foil state variables of left and right hand FABs respectively; $N_{\mathbf{p}_{\mathrm{L}}}, N_{\mathbf{p}_{\mathrm{R}}}$ denote the respective number of pads (for which the respective FD grids are $N_{\mathrm{z}_{\mathrm{L}}}$ by $N_{\theta_{\mathrm{L}}}$ and $N_{\mathrm{z}_{\mathrm{R}}}$ by $N_{\theta_{\mathrm{R}}}$ ).

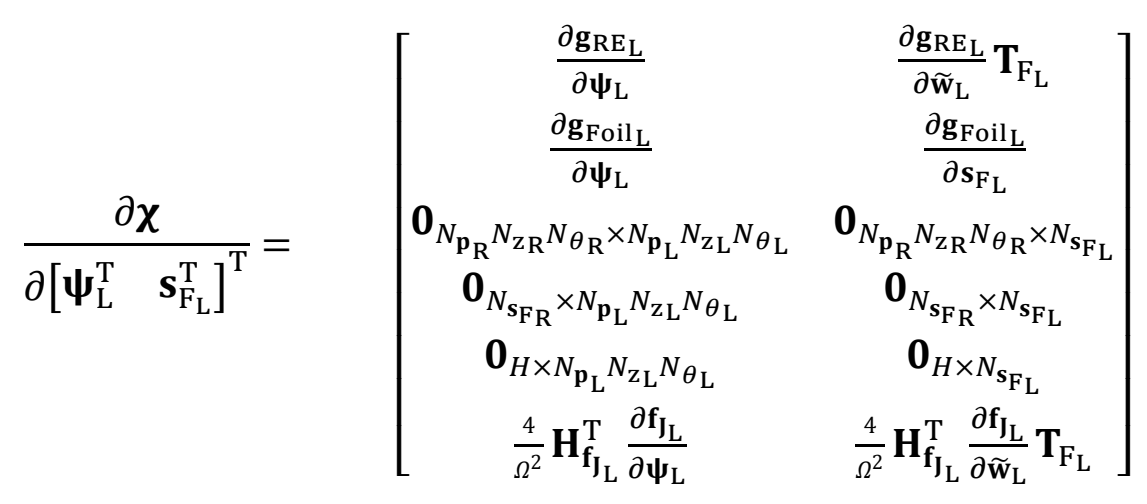

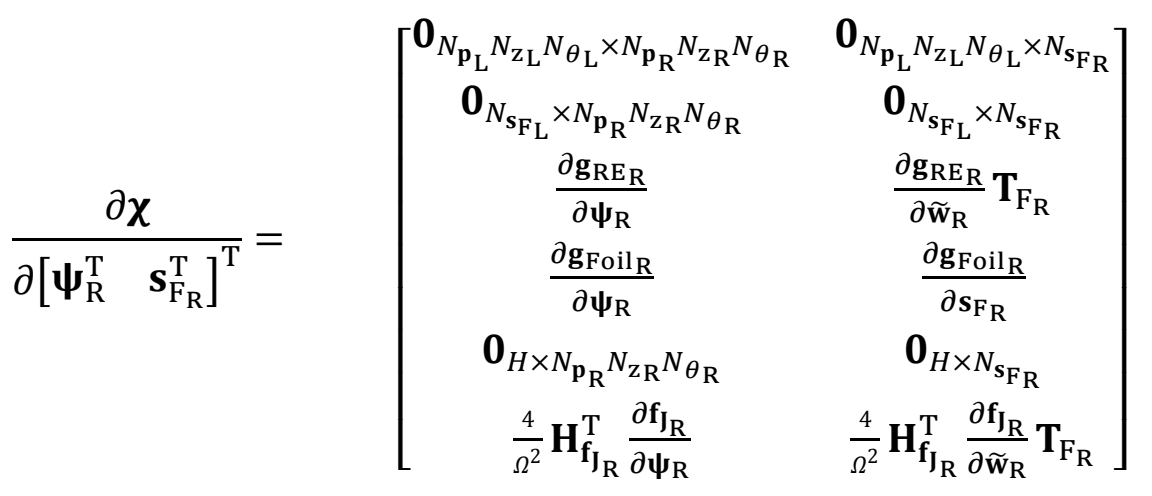




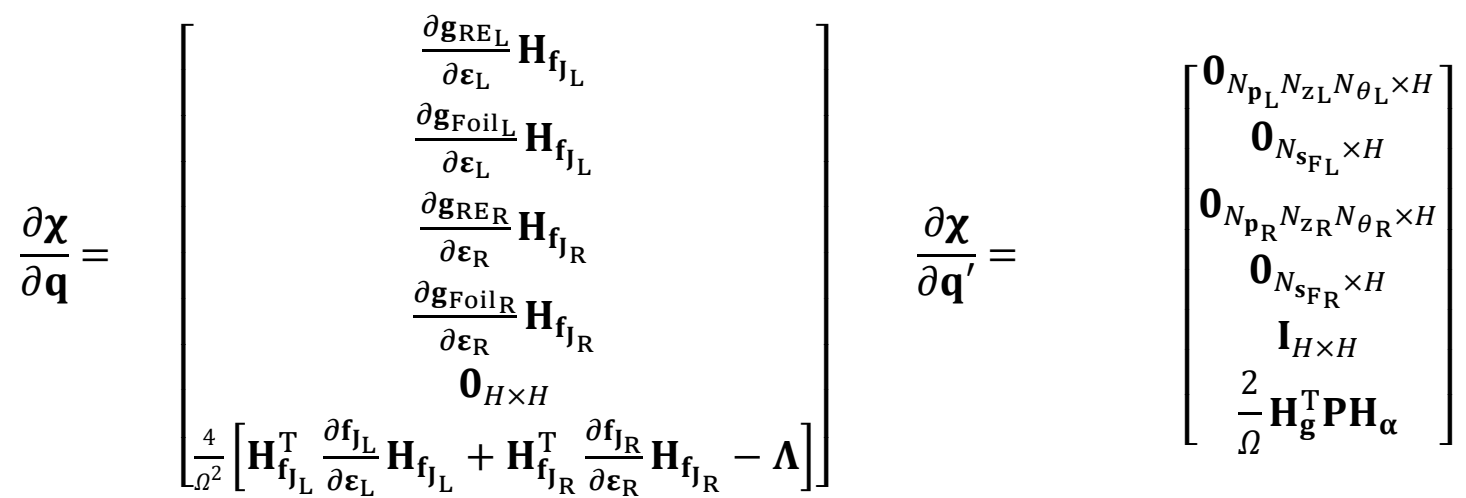

For the SEFM foil model (eq. (8)), the Jacobians of $\mathbf{g}_{\mathrm{Foil}}$ with respect to $\boldsymbol{\Psi}_{\mathrm{L}}, \mathbf{s}_{\mathrm{F}_{\mathrm{L}}}, \boldsymbol{\varepsilon}_{\mathrm{L}}$ are:

$$
\begin{aligned}
& \frac{\partial \mathbf{g}_{\mathrm{Foil}} \mathrm{L}}{\partial \boldsymbol{\Psi}_{\mathrm{L}}}=\frac{2}{\eta_{\mathrm{L}} c_{\mathrm{L}} K_{\mathrm{b}_{\mathrm{L}}}} \frac{\partial \mathbf{p}_{\mathrm{g}, \theta_{\mathrm{L}}}}{\partial \boldsymbol{\Psi}_{\mathrm{L}}} \\
& \frac{\partial \mathbf{g}_{\mathrm{Foil}_{\mathrm{L}}}}{\partial \mathbf{s}_{\mathrm{F}_{\mathrm{L}}}}=\frac{2}{\eta_{\mathrm{L}} c_{\mathrm{L}} K_{\mathrm{b}_{\mathrm{L}}}} \frac{\partial \mathbf{p}_{\mathrm{g}, \theta} \mathrm{L}}{\partial \widetilde{\mathbf{w}}_{\mathrm{L}}}-\frac{2}{\eta_{\mathrm{L}}} \mathbf{I}_{N_{\mathrm{S}_{\mathrm{L}}} \times N_{\mathrm{s}_{\mathrm{L}}}} \\
& \frac{\partial \mathbf{g}_{\mathrm{Foil}}}{\partial \boldsymbol{\varepsilon}_{\mathrm{L}}}=\frac{2}{\eta_{\mathrm{L}} c_{\mathrm{L}} K_{\mathrm{b}_{\mathrm{L}}}} \frac{\partial \mathbf{p}_{\mathrm{g}, \theta}}{\partial \boldsymbol{\varepsilon}_{\mathrm{L}}}
\end{aligned}
$$

For the FFSMM foil model (eq. (10)), the Jacobians of $\mathbf{g}_{\mathrm{Foil}}$ with respect to $\boldsymbol{\psi}_{\mathrm{L}}, \mathbf{s}_{\mathrm{F}_{\mathrm{L}}}, \boldsymbol{\varepsilon}_{\mathrm{L}}$ are:

$$
\begin{aligned}
& \frac{\partial \mathbf{g}_{\mathrm{Foil}}}{\partial \boldsymbol{\Psi}_{\mathrm{L}}}=\left[\begin{array}{c}
\mathbf{0}_{0.5 N_{\mathrm{s}_{\mathrm{F}}}} \times N_{\mathbf{p}_{\mathrm{L}}} N_{\mathrm{Z}_{\mathrm{L}}} N_{\theta_{\mathrm{L}}} \\
\frac{4}{\Omega^{2}} \mathbf{H}_{\mathbf{w}_{\mathbf{p}}}^{\mathbf{T}} \frac{\partial \mathbf{f}_{\mathbf{p}_{\mathrm{L}}}}{\partial \boldsymbol{\Psi}_{\mathrm{L}}}
\end{array}\right]
\end{aligned}
$$

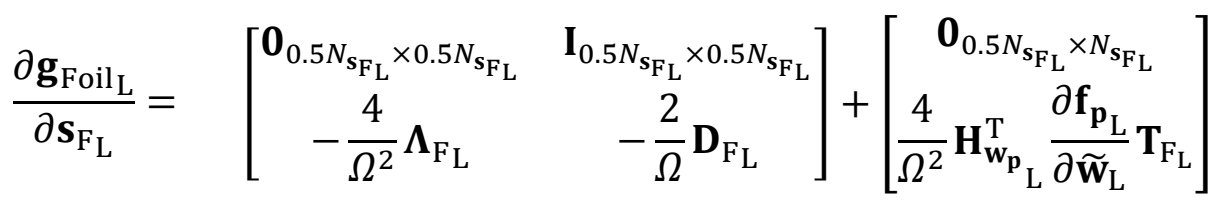

$$
\begin{aligned}
& \frac{\partial \mathbf{g}_{\text {Foil }}}{\partial \boldsymbol{\varepsilon}_{\mathrm{L}}}=\quad\left[\begin{array}{c}
\mathbf{0}_{0.5 N_{\mathrm{s}_{\mathrm{L}}}} \times 2 \\
\frac{4}{\Omega^{2}} \mathbf{H}_{\mathbf{w}_{\mathbf{p}}}^{\mathrm{T}} \frac{\partial \mathbf{f}_{\mathbf{p}_{\mathrm{L}}}}{\partial \boldsymbol{\varepsilon}_{\mathrm{L}}}
\end{array}\right]
\end{aligned}
$$

The Jacobians of $\mathbf{g}_{\mathrm{Foil}_{\mathrm{R}}}$ with respect to $\boldsymbol{\Psi}_{\mathrm{R}}, \mathbf{s}_{\mathrm{F}_{\mathrm{R}}}, \boldsymbol{\varepsilon}_{\mathrm{R}}$ are as eqs. (32) or (33) with L replaced by R. 
Upon examining eqs. (28-33) and focusing on the left hand FAB, symbolic functional expressions of $\mathbf{g}_{\mathrm{RE}_{\mathrm{L}}}, \mathbf{f}_{\mathrm{J}_{\mathrm{L}}}, \mathbf{p}_{\mathrm{g}, \theta_{\mathrm{L}}}(\mathrm{SEFM}) / \mathbf{f}_{\mathbf{p}_{\mathrm{L}}}(\mathrm{FFSMM})$, each in terms of $\left(\boldsymbol{\Psi}_{\mathrm{L}}, \widetilde{\mathbf{w}}_{\mathrm{L}}, \boldsymbol{\varepsilon}_{\mathrm{L}}\right)$, can be established based on the modelling in section 2. This is performed using a facility like the Symbolic Math Toolbox ${ }^{\mathrm{TM}}$ [36], which is then used to derive symbolic expressions for the Jacobians of these functions with respect to $\Psi_{\mathrm{L}}, \widetilde{\mathbf{w}}_{\mathrm{L}}$, $\boldsymbol{\varepsilon}_{\mathrm{L}}$. These symbolic Jacobians are then converted into subroutines. This results in considerable time savings in the case of the Jacobians with respect to $\Psi_{\mathrm{L}, \mathrm{R}}, \widetilde{\mathbf{w}}_{\mathrm{L}, \mathrm{R}}$ which have numerous columns. In the case of the Jacobians with respect to $\varepsilon_{\mathrm{L}, \mathrm{R}}$ one can alternatively apply a numerical calculation (using numjac) since these Jacobians have only two columns. In any case, if applying the Gümbel condition to $\mathbf{f}_{\mathrm{J}_{\mathrm{L}, \mathrm{R}}}$ (section 2.3.3), the Jacobians of $\mathbf{f}_{\mathrm{L}, \mathrm{R}}$ with respect to $\boldsymbol{\varepsilon}_{\mathrm{L}, \mathrm{R}}$ must be computed numerically. Also, in this case, those state variables in $\Psi_{\mathrm{L}, \mathrm{R}}, \widetilde{\mathbf{w}}_{\mathrm{L}, \mathrm{R}}$ that are associated with sub-atmospheric regions of the FD grid must be identified and the corresponding columns in the symbolic forms of $\frac{\partial \mathbf{f}_{\mathrm{J}_{\mathrm{L}}}}{\partial \Psi_{\mathrm{L}}}, \frac{\partial \mathbf{f}_{\mathrm{I}_{\mathrm{L}}}}{\partial \widetilde{\mathbf{w}}_{\mathrm{L}}}, \frac{\partial \mathbf{f}_{\mathrm{I}_{\mathrm{R}}}}{\partial \boldsymbol{\Psi}_{\mathrm{R}}}, \frac{\partial \mathbf{f}_{\mathrm{I}_{R}}}{\partial \widetilde{\mathrm{w}}_{\mathrm{R}}}$ corrected by replacing them with numerical partial derivatives. Efficient coding ensures that this additional procedure has little effect on the computation time.

\subsection{Eigenvalue Analysis, Extraction of Campbell Diagram and Associated Information}

The second part of the SESA involves the analysis of small perturbations $\Delta \mathbf{s}$ about the static equilibrium condition $\mathbf{s}=\mathbf{s}_{\mathrm{E}}$ at a given speed for no unbalance excitation, for which the dynamical system of eq. (1) can be linearised as follows:

$$
(\Delta \mathbf{s})^{\prime}=\mathbf{J}(\Delta \mathbf{s})
$$

where $\mathbf{J}$ is given by eq. (26).

\subsubsection{Eigenvalue Analysis}

The eigenvalue analysis of the matrix $\mathbf{J}$ yields $N_{\mathrm{s}}$ eigenvalues that, in the most general case, comprise $K_{\mathrm{e}}$ real eigenvalues $\lambda_{k}$ with associated eigenvectors $\gamma^{(k)}\left(k=1 \ldots K_{\mathrm{e}}\right)$ and $N_{\mathrm{o}}$ pairs of complex conjugate 
eigenvalues $\lambda_{n, \operatorname{Re}} \pm \mathrm{j} \lambda_{n, \operatorname{Im}}, \lambda_{n, \operatorname{Im}}>0$, with associated conjugate eigenvectors $\boldsymbol{\rho}^{(n)}, \boldsymbol{\rho}^{(n) *}\left(n=1 \ldots N_{\mathrm{o}}\right)$. The general solution of the perturbation equation (34) is therefore given by:

$$
\boldsymbol{\Delta} \mathbf{s}=\sum_{k=1}^{K_{\mathrm{e}}} b_{k} \boldsymbol{\gamma}^{(k)} \mathrm{e}^{\lambda_{k} \tau}+\sum_{n=1}^{N_{\mathrm{o}}} c_{n} \mathrm{e}^{\lambda_{n, \mathrm{Re}} \tau} \bmod \left\{\boldsymbol{\rho}^{(n)}\right\} \cdot * \cos \left(\lambda_{n, \mathrm{Im}} \tau+\arg \left\{\boldsymbol{\rho}^{(n)}\right\}+\alpha_{n}\right)
$$

where $b_{k}, c_{n}, \alpha_{n}$ are arbitrary real scalar constants and, for a vector $\mathbf{v}$ with complex scalar elements $\mathbf{v}=\left[\begin{array}{lll}v_{1} & \cdots & v_{N_{\mathrm{o}}}\end{array}\right]^{\mathrm{T}}:$

$$
\bmod \{\mathbf{u}\}=\left[\begin{array}{lll}
\left|v_{1}\right| & \cdots & \left|v_{N_{\mathrm{o}}}\right|
\end{array}\right]^{\mathrm{T}}, \arg \{\mathbf{v}\}=\left[\begin{array}{lll}
\arg \left\{v_{1}\right\} & \cdots & \arg \left\{v_{N_{\mathrm{o}}}\right\}
\end{array}\right]^{\mathrm{T}}
$$

and the operation .* in eq. (35) denotes term-by-term multiplication of two vectors $\mathbf{a}, \mathbf{b}$

$$
\mathbf{a}=\left[\begin{array}{lll}
a_{1} & \cdots & a_{N_{\mathrm{o}}}
\end{array}\right]^{\mathrm{T}}, \mathbf{b}=\left[\begin{array}{lll}
b_{1} & \cdots & b_{N_{\mathrm{o}}}
\end{array}\right]^{\mathrm{T}}, \mathbf{a} \cdot * \mathbf{b}=\left[\begin{array}{lll}
a_{1} b_{1} & \cdots & a_{N_{\mathrm{o}}} b_{N_{\mathrm{o}}}
\end{array}\right]^{\mathrm{T}}
$$

The stability of the perturbation is governed by the leading eigenvalue $\lambda_{\mathrm{L}}$, which is one with highest real part:

$$
\operatorname{Re}\left\{\lambda_{\mathrm{L}}\right\}=\max \left(\lambda_{1}, \quad \cdots \quad, \lambda_{K_{\mathrm{e}}}, \lambda_{1, \mathrm{Re}}, \quad \cdots \quad, \lambda_{N_{\mathrm{o}}, \mathrm{Re}}\right)
$$

From eq. (35), transforming from non-dimensional time $\tau=\Omega t / 2$ to time $t$ in seconds:

$$
\varpi_{\mathrm{d}_{\mathrm{L}}} / \Omega=\left|\operatorname{Im}\left\{\lambda_{\mathrm{L}}\right\}\right| / 2
$$

where $\varpi_{d_{L}}(\mathrm{rad} / \mathrm{s})$ is the circular frequency of the whirl component of eq. (35) that is associated with the leading eigenvalue $\lambda_{\mathrm{L}}$.

As in [1], the onset of instability (OIS) speed is defined as the first speed, in discrete steps, to register a leading eigenvalue $\lambda_{\mathrm{L}}$ with positive real part (Hopf bifurcation [2, 4]). 
The expression in eq. (35) is more conveniently expressed in terms of $t$ as follows:

$$
\boldsymbol{\Delta} \mathbf{s}=\sum_{k=1}^{K_{\mathrm{e}}} b_{k} \boldsymbol{\gamma}^{(k)} \mathrm{e}^{\left(\frac{\Omega}{2} \lambda_{k}\right) t}+\sum_{n=1}^{N_{\mathrm{o}}} c_{n} \mathrm{e}^{-\zeta_{n} \varpi_{\mathrm{u}, n} t} \bmod \left\{\boldsymbol{\rho}^{(n)}\right\} \cdot * \cos \left(\varpi_{\mathrm{d}, n} t+\arg \left\{\boldsymbol{\rho}^{(n)}\right\}+\alpha_{n}\right)
$$

where the $\varpi_{\mathrm{d}, n}, \zeta_{n}, \varpi_{\mathrm{u}, n}$ are, respectively, the damped natural circular frequency, equivalent viscous damping ratio, and undamped natural circular frequency of (oscillatory) mode no. $n$ :

$$
\varpi_{\mathrm{d}, n}=\frac{\Omega}{2} \lambda_{n, \mathrm{Im}}, \zeta_{n}=\frac{-\lambda_{n, \mathrm{Re}}}{\sqrt{\lambda_{n, \mathrm{Re}}^{2}+\varpi_{\mathrm{d}, n}^{2}}}, \varpi_{\mathrm{u}, n}=\frac{\varpi_{\mathrm{d}, n}}{\sqrt{1-\zeta_{n}^{2}}}
$$

It is important to emphasise that these modes pertain to small linearised perturbations about the static equilibrium state $\mathbf{S}=\mathbf{s}_{\mathrm{E}}$ of the nonlinear coupled rotor-FAB system (eq. (1)) at a given rotational speed $\Omega$ and should not be confused with component modes (used in eqs. (2e)).

The number of oscillatory modes $N_{\mathrm{o}}$ at each given rotational speed $\Omega$ is typically very high since the dimension of the Jacobian is very high. As an illustration of this, the reader is referred to the eigenfrequency vs speed map shown in Figure 9, where, for a given speed, each dot that is not on the horizontal axis is one of the $N_{\mathrm{o}}$ oscillatory modes in eq. (40). This graph belongs to the test rig rotor of Case no. 1 in Table 2. As shall be revealed later, most of these dots do not represent any significant vibration (whirl) of the rotor and so, the graph in Figure 9 cannot be the Campbell diagram. The Campbell diagram of the FAB-rotor system is in fact "buried" within the eigenfrequency vs speed map in Figure 9 and needs to be extracted by applying a filtering criterion.

\subsubsection{Extraction of Campbell Diagram and Associated Information}


The eigenvectors $\boldsymbol{\rho}^{(n)}$ first need to be scaled by an appropriate factor $\kappa^{(n)}$. This factor is chosen so that, for mode no. $n$, the overall greatest non-dimensional amplitude of vibration within the FABs (comparing journal vibration with foil pad vibration, and considering both "L" and "R" FABs) is a prescribed fraction $\sigma$ of the nominal radial clearance of the FAB where it occurs. The scaling factor that will fulfil this requirement is

$$
\kappa^{(n)}=\sigma / \tilde{A}^{(n)}
$$

where $\tilde{A}^{(n)}$ is the overall greatest non-dimensional vibration amplitude within the two FABs:

$$
\tilde{A}^{(n)}=\max \left(\bmod \left\{\boldsymbol{\rho}_{\boldsymbol{\varepsilon}_{\mathrm{L}}}^{(n)}\right\}, \bmod \left\{\boldsymbol{\rho}_{\widetilde{\mathbf{w}}_{\mathrm{L}}}^{(n)}\right\}, \bmod \left\{\boldsymbol{\rho}_{\boldsymbol{\varepsilon}_{\mathrm{R}}}^{(n)}\right\}, \bmod \left\{\boldsymbol{\rho}_{\widetilde{\mathbf{w}}_{\mathrm{R}}}^{(n)}\right\}\right)
$$

where $\boldsymbol{\rho}_{\varepsilon_{\mathrm{L}}}^{(n)}, \boldsymbol{\rho}_{\widetilde{\mathbf{w}}_{\mathrm{L}}}^{(n)}, \boldsymbol{\rho}_{\varepsilon_{\mathrm{R}}}^{(n)}, \boldsymbol{\rho}_{\widetilde{\mathbf{w}}_{\mathrm{R}}}^{(n)}$ are the complex amplitudes of the damped harmonic perturbations $\Delta \boldsymbol{\varepsilon}_{\mathrm{L}}^{(n)}$, $\Delta \widetilde{\mathbf{w}}_{\mathrm{L}}^{(n)}, \Delta \boldsymbol{\varepsilon}_{\mathrm{R}}^{(n)}, \Delta \widetilde{\mathbf{w}}_{\mathrm{R}}^{(n)}$ respectively, which, from eq. (40), are given by:

$$
\begin{aligned}
& \Delta \boldsymbol{\varepsilon}_{\mathrm{L}, \mathrm{R}}^{(n)}(t)=c_{n} \mathrm{e}^{-\zeta_{n} \varpi_{\mathrm{u}, n} t} \bmod \left\{\boldsymbol{\rho}_{\varepsilon_{\mathrm{L}, \mathrm{R}}}^{(n)}\right\} * \cos \left(\varpi_{\mathrm{d}, n} t+\arg \left\{\boldsymbol{\rho}_{\varepsilon_{\mathrm{L}, \mathrm{R}}}^{(n)}\right\}+\alpha_{n}\right) \\
& \Delta \widetilde{\mathbf{w}}_{\mathrm{L}, \mathrm{R}}^{(n)}(t)=c_{n} \mathrm{e}^{-\zeta_{n} \varpi_{\mathrm{u}, n} t} \bmod \left\{\boldsymbol{\rho}_{\widetilde{\mathbf{w}}_{\mathrm{L}, \mathrm{R}}}^{(n)}\right\} * \cos \left(\varpi_{\mathrm{d}, n} t+\arg \left\{\boldsymbol{\rho}_{\widetilde{\mathbf{w}}_{\mathrm{L}, \mathrm{R}}}^{(n)}\right\}+\alpha_{n}\right)
\end{aligned}
$$

From eqs. (6), (7):

$$
\boldsymbol{\rho}_{\varepsilon_{\mathrm{L}, \mathrm{R}}}^{(n)}=\mathbf{H}_{\mathrm{f}_{\mathrm{L}, \mathrm{R}}} \boldsymbol{\rho}_{\mathbf{q}}^{(n)} / c_{\mathrm{L}, \mathrm{R}}, \quad \boldsymbol{\rho}_{\widetilde{\mathbf{w}}_{\mathrm{L}, \mathrm{R}}}^{(n)}=\mathbf{T}_{\mathrm{F}_{\mathrm{L}}} \boldsymbol{\rho}_{\mathbf{s}_{\mathrm{L}, \mathrm{R}}}^{(n)}
$$

where the complex vectors $\boldsymbol{\rho}_{\mathbf{q}}^{(n)}, \boldsymbol{\rho}_{\mathbf{s}_{\mathrm{F}, \mathrm{R}}}^{(n)}$ are obtained by partitioning the eigenvector $\boldsymbol{\rho}^{(n)}$ into its constituent sub-vectors according to the state vector $\mathbf{s}$ (eq. (3)):

$$
\boldsymbol{\rho}^{(n)}=\left[\begin{array}{c}
\boldsymbol{\rho}_{\boldsymbol{\Psi}_{\mathrm{L}}}^{(n)} \\
\boldsymbol{\rho}_{\mathbf{s}_{\mathrm{F}_{\mathrm{L}}}}^{(n)} \\
\boldsymbol{\rho}_{\boldsymbol{\Psi}_{\mathrm{R}}}^{(n)} \\
\boldsymbol{\rho}_{\mathbf{s}_{\mathrm{F}_{\mathrm{R}}}}^{(n)} \\
\boldsymbol{\rho}_{\mathbf{q}}^{(n)} \\
\boldsymbol{\rho}_{\mathbf{q}^{\prime}}^{(n)}
\end{array}\right]
$$


The scaled eigenvectors are denoted by $\tilde{\boldsymbol{\rho}}^{(n)}$ where

$$
\tilde{\boldsymbol{\rho}}^{(n)}=\kappa^{(n)} \boldsymbol{\rho}^{(n)}
$$

The vectors derived according to eqs. $(46,47)$ using the sub-vectors of the scaled eigenvectors $\tilde{\boldsymbol{\rho}}^{(n)}$ are given similar notation i.e. $\widetilde{\boldsymbol{\rho}}_{\varepsilon_{\mathrm{L}, \mathrm{R}}}^{(n)}, \widetilde{\boldsymbol{\rho}}_{\widetilde{\mathbf{w}}_{\mathrm{L}, \mathrm{R}}}^{(n)}$,...etc. and the result of eq. (43) using these vectors becomes $\tilde{A}^{(n)}=\sigma$.

Let $\tilde{A}_{\varepsilon_{\mathrm{L}}}^{(n)}, \tilde{A}_{\varepsilon_{\mathrm{R}}}^{(n)}$ denote the mean of the $x, y$ non-dimensional scaled vibration amplitudes of the journals of the L, R FABs respectively:

$$
\tilde{A}_{\boldsymbol{\varepsilon}_{\mathrm{L}}}^{(n)}=\operatorname{mean}\left(\bmod \left\{\tilde{\boldsymbol{\rho}}_{\boldsymbol{\varepsilon}_{\mathrm{L}}}^{(n)}\right\}\right), \tilde{A}_{\boldsymbol{\varepsilon}_{\mathrm{R}}}^{(n)}=\operatorname{mean}\left(\bmod \left\{\tilde{\boldsymbol{\rho}}_{\boldsymbol{\varepsilon}_{\mathrm{R}}}^{(n)}\right\}\right)
$$

The filtering criterion to be adopted is that mode no. $n$ will be rejected from the Campbell diagram unless at least one of $\tilde{A}_{\varepsilon_{\mathrm{L}}}^{(n)}, \tilde{A}_{\mathcal{\varepsilon}_{\mathrm{R}}}^{(n)}$ is greater than $C$ times $\sigma$, where $C$ is a cut-off threshold. Hence, going through the series in the second term (i.e. the oscillatory part) of eq. (40), a mode is only accepted into the Campbell diagram if the following condition is satisfied:

$$
\left(\tilde{A}_{\mathcal{\varepsilon}_{\mathrm{L}}}^{(n)}>C \sigma\right) \operatorname{OR}\left(\stackrel{m}{A}_{\boldsymbol{\varepsilon}_{\mathrm{R}}}^{(n)}>C \sigma\right)
$$

In this work, $\sigma=0.2$ and $C=0.1$. The eigenvectors are therefore scaled such that the greatest overall non-dimensional amplitude of vibration in the FABs is $20 \%$ (of the nominal radial clearance of the FAB where it occurs). The scaled mode is then excluded from the Campbell diagram if both FABs have mean $x, y$ journal vibration amplitudes that are less than (or equal to) $2 \%$ (i.e. 0.1 of 0.2 ) of their respective nominal radial clearances.

The application of the filtering criterion defined by the condition in (51) to the raw eigenfrequency $v s$ speed map of Figure 9 results in the emergence of the clearly defined Campbell diagram in Figure 10. The Campbell diagram is seen to have 4 whirl modes at each speed, which correlates with the fact that the 
test rig rotor model of Case no. 1 (Table 2) is a rigid rotor with 4 degrees of freedom. Examples of modes that were rejected shall be given later on. As will be shown in the results sections (sections 4,5 ), it may also be appropriate in some situations to apply another criterion based on maximum allowable modal damping ratio $\left(\zeta_{n}\right.$ in eq. (40)) in conjunction with the above minimum journal amplitude criterion of (51).

Each point on the Campbell diagram in Figure 10 also indicates the stability of the mode and its direction of whirl. The latter is determined from the mode shape of the rotor that is derived as follows.

Considering eqs. (4) and (40), the 3D-shape of the rotor in mode no. $n$ at speed $\Omega$ is defined by the orbit of generic point $\mathrm{P}$ along the rotor centreline (at axial coordinate $z$ ) about its static equilibrium position at $\Omega$ :

$$
\begin{aligned}
& \mathbf{v}_{\mathrm{P}}^{(n)}(z, t)=\left[\begin{array}{c}
x_{\mathrm{P}}^{(n)}(z, t) \\
y_{\mathrm{P}}^{(n)}(z, t)
\end{array}\right]=\left[\begin{array}{l}
x_{\mathrm{P}_{\mathrm{E}}}(z) \\
y_{\mathrm{P}_{\mathrm{E}}}(z)
\end{array}\right]+\left[\begin{array}{l}
\Delta x_{\mathrm{P}}^{(n)}(z, t) \\
\Delta y_{\mathrm{P}}^{(n)}(z, t)
\end{array}\right] \\
& \Rightarrow \\
& \mathbf{v}_{\mathbf{P}}^{(n)}(z, t)=\left[\begin{array}{l}
x_{\mathbf{P}}^{(n)}(z, t) \\
y_{\mathbf{P}}^{(n)}(z, t)
\end{array}\right]=\mathbf{H}_{\mathbf{v}_{\mathbf{P}}} \mathbf{q}_{\mathbf{E}}+c_{n} \mathrm{e}^{-\zeta_{n} \varpi_{\mathrm{u}, n} t} \bmod \left\{\mathbf{H}_{\mathbf{v}_{\mathbf{P}}} \hat{\boldsymbol{\rho}}_{\mathbf{q}}^{(n)}\right\} \cdot \cos \left(\varpi_{\mathrm{d}, n} t+\arg \left\{\mathbf{H}_{\mathbf{v}_{\mathbf{P}}} \hat{\boldsymbol{\rho}}_{\mathbf{q}}^{(n)}\right\}+\alpha_{n}\right)
\end{aligned}
$$

where $\mathbf{q}_{\mathbf{E}}$ is the $\mathbf{q}$ component of the static equilibrium solution $\mathbf{s}=\mathbf{s}_{\mathbf{E}}$ (partitioned as per eq. (3)). The 3D-mode shape is traced by plotting eq. (53) at a number of discrete times covering the period $2 \pi / \varpi_{\mathrm{d}, n}$, from which the direction of whirl (forward or reverse) can be determined.

The results of the above novel Campbell diagram analysis for any specific mode can be correlated with the full nonlinear dynamical system (eq. (1)) through the novel approach of mode-specific initial conditions for TNDA. The initial conditions to induce the dynamical system to respond in one specific mode only (with no unbalance excitation applied) are given by:

$$
\mathbf{s}(\tau=0)=\mathbf{s}_{\mathbf{E}}+c_{n} \bmod \left\{\tilde{\boldsymbol{\rho}}^{(n)}\right\} \cdot * \cos \left(\arg \left\{\tilde{\boldsymbol{\rho}}^{(n)}\right\}+\alpha_{n}\right)
$$


where the arbitrary scalar phase $\alpha_{n}$ can be set to zero and the factor $c_{n}$ set to any convenient fraction $\left(c_{n}=0.1\right.$ in this work - this is, in fact, a further reduction factor that is over and above the reduction involved in the scaling the eigenvectors as per eq. (49)).

\section{PRESENTATION OF RESULTS AND DISCUSSION: CASE No. 1 (TABLE 2)}

From [14, 11, 10], for Case No. 1 (Table 2), the parameters of the 3-pad FABs (Figure 2(a)) are as follows (L, R bearings are identical): $c_{\mathrm{L}}=40 \times 10^{-6} \mathrm{~m} ; \alpha^{(k)}=115 \pi / 180 ; h_{\mathrm{s}}^{(k)}=50 \times 10^{-6} \mathrm{~m}$; $\beta^{(k)}=30 \pi / 180 ; \theta_{\mathrm{st}}^{(k)}=30 \pi / 180+(k-1) 2 \pi / 3+\pi / 2 ; R_{\mathrm{L}}=33.5 \times 10^{-3} \mathrm{~m} ; L_{\mathrm{L}}=53 \times 10^{-3} \mathrm{~m} ;$ viscosity $\mu=1.95 \times 10^{-5} \mathrm{~Pa} \cdot \mathrm{s} ; p_{\mathrm{a}}=100 \times 10^{3} \mathrm{~Pa}$; damping loss factor $\eta_{\mathrm{L}}=0.15$. In all cases, $K_{\mathrm{b}_{\mathrm{L}}}=K_{\mathrm{b}_{\mathrm{R}}}=K_{\mathrm{b}}$

With regard to Figure $3, l_{\mathrm{L}}=0.1979 \mathrm{~m}, l_{\mathrm{R}}=0.2011 \mathrm{~m}$, rotor mass $M_{\text {tot }}=21.1166 \mathrm{~kg}$, diametral moment of inertia (MoI) about $\mathrm{G}, I_{\mathrm{G}}=525.166 \times 10^{-3} \mathrm{kgm}^{2}$; polar MoI $I_{\mathrm{p}}=30.079 \times 10^{-3} \mathrm{kgm}^{2}[14$, $11,10]$. The rigid rotor equations in $[14,11,10]$ were not expressed in the generic component mode synthesis format (section 2.1), but the system can be expressed in this form by considering a total of $H=4$ modes of the free-free rotor, comprising two modes at $0 \mathrm{~Hz}$ in each of the $x z$ and $y z$ planes, one mode $\varphi_{\text {trans }}(z)$ defining pure translation, the other $\varphi_{\text {rot }}(z)$ defining pure rotation about G:

$$
\varphi_{\text {trans }}(z)=1 / \sqrt{M_{\text {tot }}}, \quad \varphi_{\text {trans }}(z)=\left(z-l_{\mathrm{L}}\right) / \sqrt{I_{\mathrm{G}}}
$$

where $z$ is the distance from the L FAB. All modal matrices in eq. (2e) can be constructed using eqs. (55a,b) and their slopes (for the gyroscopic terms). Modal data and other data for this case, and the other two cases in Table 2, are supplied as datasets linked to this paper.

Unless otherwise stated, the FD grid for each pad of the FAB is $7 \times 49$, which is equivalent to a $15 \times 51$ grid covering the entire pad considering symmetry about the vertical mid-section of the bearing and including the edges. The $7 \times 49$ FD grid was chosen after a mesh dependency study (section 4.2). The onset of 
instability (OIS) speed is defined as the first speed, in steps of $500 \mathrm{rpm}$ (reduced to $100 \mathrm{rpm}$ if appropriate), to show instability.

\subsection{Correlation with TNDA Results of [10]}

The modelling technique used in this paper was first correlated with that of $[14,11,10]$, using the results published in [10], where the bump foil stiffness was $K_{\mathrm{b}_{\mathrm{L}}}=K_{\mathrm{b}_{\mathrm{R}}}=9.26 \times 10^{9} \mathrm{~N} / \mathrm{m}^{3}$. Figures $4(\mathrm{a}, \mathrm{b})$ show, for a speed of $20 \mathrm{krpm}$, the TNDA simulations of the trajectory of R FAB journal centre at zero rotor unbalance at default initial conditions (zero journal displacement and velocity, air film at atmospheric pressure, pads undeformed). For both models, the trajectory converges to a static equilibrium position. Figures $4(\mathrm{c}, \mathrm{d})$ repeat the same for a speed of $30 \mathrm{krpm}$. For both models, the trajectory diverges. In fact the OIS was computed by SESA to be $30 \mathrm{krpm}$ for $K_{\mathrm{b}_{\mathrm{L}}}=K_{\mathrm{b}_{\mathrm{R}}}=9.26 \times 10^{9}$ $\mathrm{N} / \mathrm{m}^{3}$. The trajectories of both models (Figures 4(a,c) vs Figures 4(b,d)) are considered satisfactorily similar, despite visible differences. Such differences are explicable by the different modelling techniques for the air film (FD vs Bubnov-Galerkin FE) and subtle differences in modelling the pad contour. As a further check, the steady-state unbalance response at the stable speed of $20 \mathrm{krpm}$ was computed by TNDA for two levels of unbalance and compared with the results in [10]. With $20 \mathrm{~g} \cdot \mathrm{mm}$ applied at each bearing in anti-phase, the response frequency spectra of both models exhibit the synchronous component only (Figures 5(a,b)). As the unbalance level at each bearing is doubled, both models predict the appearance of non-synchronous frequencies of significant strength at similar locations on the frequency spectrum (Figures 6(a,b)).

\section{Figure 4}

\section{Figure 5}

\section{Figure 6}




\subsection{Campbell Diagram Analysis Using Parameters of [14]}

In this section, the three stiffness values in Table 1 are used for $K_{\mathrm{b}}\left(=K_{\mathrm{b}_{\mathrm{L}}}=K_{\mathrm{b}_{\mathrm{R}}}\right)$. The OIS values computed by SESA for the three cases in Table 1 were $19.5 \mathrm{krpm}, 30.5 \mathrm{krpm}, 33.6 \mathrm{krpm}$ respectively, the corresponding dominant whirl frequencies being $97.33 \mathrm{~Hz}, 97.22 \mathrm{~Hz}, 90.51 \mathrm{~Hz}$. Comparing with the TNDA values in the third and sixth columns of Table 1 , the discrepancies were $0.3 \%, 5.5 \%, 7 \%$ for OIS and $1.5 \%, 1.4 \%, 0.1 \%$ for the dominant whirl frequencies. These discrepancies are again attributed to the different modelling techniques. Nonetheless, the discrepancies in OIS are much lower than the OIS discrepancies in Table 1 (as reported by [14]) for the linearised method (Lund's) relative to TNDA where both methods were based on the same model (Bubnov-Galerkin FE for air film). Significantly, for a given model (FD for air film), the author's linearised method (SESA) has virtually zero error relative to TNDA. This was already shown for the dominant whirl mode for other cases e.g. $[1,5,6]$, and will be shown for each mode on the Campbell diagram in the following subsection, which focuses on the design stiffness value in Table 1 i.e. $8.8 \times 10^{9} \mathrm{~N} / \mathrm{m}^{3}$, that is $5 \%$ lower than in section 4.1 and $2 \%$ lower than used in the experimental work in [10].

\subsubsection{Results for $K_{\mathrm{b}}=8.8 \times 10^{9} \mathrm{~N} / \mathrm{m}^{3}$ (Design Stiffness)}

Figure 7 shows the results of a study showing the dependency of the leading eigenvalue $\lambda_{\mathrm{L}}$ (eqs. (38), (39)) on the FD grid size. Increasing the grid size per pad from $7 \times 23$ to $7 \times 49$ (i.e. adding more points in the $\theta$ direction) shifted the OIS from $31 \mathrm{krpm}$ to $30.5 \mathrm{krpm}$. Raising the grid size to $15 \times 49$ did not affect the OIS. Hence, a grid size of $7 \times 49$ was considered appropriate.

\section{Figure 7}

Figure 8 compares the leading eigenvalue of the Jacobian computed by the fully numerical method using numjac.m [3] with that of the Jacobian computed by the part-analytical method of section 3.1.1. As can be seen, the agreement is very good. For each speed the calculation of $\lambda_{\mathrm{L}}$ involves two parts: (a) process 
no. 2 in section 3.1 where the Jacobian $\mathbf{J}$ is computed by one of the two methods; (b) process no. 3 in section 3.1 involving the eigenvalue analysis of $\mathbf{J}$. Use of the part-analytical method reduced the cumulative time for step (a) over the full speed range by $56 \%$. However, this translated to a time saving of $28 \%$ when the total cumulative time for both steps (a) and (b) was considered - on a desktop PC running Matlab R2012a the total time using the part-analytical method was 19.4 mins compared to a total time of 26.9 mins using the full numerical method. The reason is that step (b) occupies a large portion of the time due to the size of $\mathbf{J}$, which is $2360 \times 2360$ for a $7 \times 49$ grid per pad, since, from eq. (3), the length of $\mathbf{s}$ is $N_{\mathrm{s}}=2(3 \times 7 \times 49+3 \times 49)+2 \times 4$.

\section{Figure 8}

Figure 9 shows the unfiltered eigenfrequency $f_{\mathrm{d}, n}\left(=\varpi_{\mathrm{d}, n} /(2 \pi)\right) v s$ speed map. For each given speed at or beyond the OIS, only one dot per speed represents an unstable mode. Figure 10 shows the Campbell diagram extracted from Figure 9 using the minimum journal amplitude criterion, eq. (51), with $C=0.1$. The modes ("mode points") at individual speeds each lie on a distinct curve covering the speed range. These curves are labelled "mode 1", "mode 2", ... etc. The 1 EO line intersects with modes 1, 2 and 4 at $83 \mathrm{~Hz}, 104 \mathrm{~Hz}$ and $194 \mathrm{~Hz}$. The latter two are 5.8\%, 8.2\% different from the approximate critical speeds $(110 \mathrm{~Hz}, 210 \mathrm{~Hz})$ observed in [11] from the waterfall diagram of the steady-state TNDA response at residual unbalance covering the speed range $90-440 \mathrm{~Hz}$ in steps of $5 \mathrm{~Hz}$. Figure 10 also shows that the 1 EO line intersects with mode 3 , but the intersection point is a reverse whirl mode and such modes are not normally excited by rotational unbalance [7].

\section{Figure 9}

\section{Figure 10}

Figure 11 shows two examples of modes rejected by the minimum journal amplitude criterion. These modes are characterised by significant vibration at one of the pads of either the left or right hand FAB and negligible journal vibration. These modes are definitely oscillatory (as evident by their non-zero 
eigenfrequencies), but have very high damping ratios. Those oscillatory modes in Figure 9 that failed the minimum journal amplitude criterion (eq. (51)) with $C=0.1$ were found to have damping ratios $\zeta_{n} \geq$ 0.3 .

\section{Figure 11}

Figures 12-15 respectively show the results for modes 1, 2, 3, 4 of Figure 10 as computed by the eigenvalue analysis of section 3.2, at a speed of $30 \mathrm{krpm}$, which is just below the OIS (30.5 krpm). Modes 1 and 3 both define translational rotor modes ("cylindrical" mode shape with a narrow elliptical cross-section) that are respectively forward and reverse whirl. Modes 2 and 4 both define pitching modes ("conical" mode shape with narrow elliptical cross-section) that are respectively reverse and forward whirl. It is noted from Figure 10 that mode 2 transitions from forward to reverse whirl as the speed increases beyond $14.5 \mathrm{krpm}$. At this transition speed, the ellipse in Figure 13(a) degenerates into a straight line.

\section{Figure 12}

\section{Figure 13}

\section{Figure 14}

\section{Figure 15}

The precision of the Campbell diagram results in Figures 12-15 was confirmed by TNDA using modespecific initial conditions (eq, (54)), and the results are shown in Figure 16, where Figure 16(a,b) (mode 1 specific) verifies Figure 12, Figure 16(c,d) (mode 2 specific) verifies Figure 13,...etc. The trajectories in Figures 16(a,c,e,g) confirm the whirl directions in Figures 12(a), 13(a), 14(a), 15(a) respectively. For each time history in Figures $16(\mathrm{~b}, \mathrm{~d}, \mathrm{f}, \mathrm{h}), f_{\mathrm{d}, n}$ and $\zeta_{n}$ were determined by calculating the period and applying the logarithmic decrement method [37] and the results confirmed the eigenvalue analysis values for $f_{\mathrm{d}, n}$ and $\zeta_{n}$ (eqs. $\left.(41 \mathrm{a}, \mathrm{b})\right)$ given in the captions of Figures 12-15. 


\section{Figure 16}

Figure 17 shows the TNDA result at $30.5 \mathrm{krpm}$ (OIS) for mode 1 specific initial conditions. The trajectory and time history diverge as predicted by the sign of the Campbell diagram analysis damping ratio for mode $1\left(\zeta_{n}=-0.0001252\right)$, and the frequency spectrum (Figure 17(c)) confirms the eigenfrequency.

\section{Figure 17}

It is also interesting to report how the TNDA journal trajectory evolves when starting from initial conditions that are specific to a stable mode (e.g. mode 4) at speeds that are unstable due to the instability of mode 1 . At the OIS, where the degree of instability $\left(\left|\zeta_{n}\right|\right)$ of mode 1 is very low, it is possible for the trajectory to find its way to the static equilibrium position along mode 4. However, at higher speeds (e.g. $35 \mathrm{krpm}$ ), where the degree of instability of mode 1 is considerably higher, the trajectory initially converges along mode 4 but then starts diverging along mode 1 . Similar behavior was observed by the author in a much simpler system [31].

4.2.2 Results for $K_{\mathrm{b}}=4.4 \times 10^{9} \mathrm{~N} / \mathrm{m}^{3}$ and $K_{\mathrm{b}}=8.8 \times 10^{16} \mathrm{~N} / \mathrm{m}^{3}$

Figure 18 shows the Campbell diagram for the system with low stiffness pad FABs, where the frequencies are typically lower at all speeds. It is noted that the dominant instability shifts from mode 1 to mode 4 at $35 \mathrm{krpm}$.

\section{Figure 18}

Of more interest is the case for the system with rigid pad FABs $\left(K_{\mathrm{b}}=8.8 \times 10^{16} \mathrm{~N} / \mathrm{m}^{3}\right)$. As can be seen from Figure 19, the minimum journal amplitude criterion (eq. (51)) fails to extract the Campbell diagram from the unfiltered eigenfrequency $v s$ speed map since this criterion relies on a comparison between the journal and foil amplitudes and the latter are invariably negligible relative to the journal (since the pad is rigid). Hence, an alternative filtering criterion is needed. Based on the observation made in the previous section that the unwanted modes tend to have high damping ratios, it was decided to apply a maximum 
damping ratio filtering criterion to the map in Figure 19. By imposing the condition $\zeta_{n}<0.8$, the Campbell diagram in Figure 20 was extracted. The black dots at low speeds represent isolated modes that can be removed if the maximum damping ratio is lowered to 0.7 . The diagram in Figure 20 is consistent with those of Figures 18 and 10 since the frequencies in Figure 20 are higher at all speeds. The rotor mode shapes are also similar to those in Figures 12-15.

\section{Figure 19}

\section{Figure 20}

\section{PRESENTATION OF RESULTS AND DISCUSSION: CASE No. 2 (TABLE 2)}

Case no.2 (Table 2) refers to the symmetric rigid rotor in [29], with total mass $M_{\text {tot }}=2 \times 3.061 \mathrm{~kg}$. The total number of rotor component modes is $H=2$ modes, comprising one mode at $0 \mathrm{~Hz}$ defined by $\varphi_{\text {trans }}(z)$ (eq. (55a)) in each of the $x z$ and $y z$ planes. For the two identical FABs, $c_{\mathrm{L}}=32 \times 10^{-6} \mathrm{~m}$; $R_{\mathrm{L}}=19.05 \times 10^{-3} \mathrm{~m} ; L_{\mathrm{L}}=38.1 \times 10^{-3} \mathrm{~m}$; viscosity $\mu=1.95 \times 10^{-5} \mathrm{~Pa} \cdot \mathrm{s} ; p_{\mathrm{a}}=101325 \mathrm{~Pa}$. For the FFSMM, the 5 component modes of the bump foil are those depicted in Figures 5(b1-b5) of reference [29], whose frequencies are: $1.9076 \mathrm{kHz}, 5.6798 \mathrm{kHz}, 9.3268 \mathrm{kHz}, 12.7785 \mathrm{kHz}, 15.9815 \mathrm{kHz}$. The modal damping ratio of each mode was set to 0.125 [29].

Figure 21 shows the unfiltered eigenfrequency $v s$ speed map. In addition to the dense accumulation of dots (eigenmodes) encountered in the previous section (using SEFM), one observes five high frequency modes at each speed that are introduced by the inertia of the bump foil. Three of these modes are depicted in Figure 22 at $15 \mathrm{krpm}$, where it is seen that the journal vibration is negligible. These correspond to the foil component modes but are at somewhat higher frequencies since they include the coupling of the air film which introduces a distributed radial constraint. It is noted that with the FFSMM the foil deflection is based entirely on the bump foil i.e. top foil detachment is not considered and the foil profile is based on an interpolation through the apexes of the bumps. For this reason, in Figure 22 (and 
Figure 24), the radial deflection is minimal towards the free end of the pad in accordance with bump foil component modes presented in [29].

\section{Figure 21}

\section{Figure 22}

The application of the minimum journal amplitude criterion, eq. (51), with $C=0.1$ results in the extraction of the Campbell diagram in Figure 23, for which the modes at $15 \mathrm{krpm}$ are depicted in Figures 24(a-d). Only two of the modes in the Campbell diagram are of practical significance - mode 1 (forward whirl) and mode 2 (reverse whirl). The other two modes are highly damped and can be filtered out by applying an additional maximum damping criterion of $\zeta_{n}<0.7$.

\section{Figure 23}

\section{Figure 24}

\section{PRESENTATION OF RESULTS AND DISCUSSION: CASE No. 3 (TABLE 2)}

Case no.3 (Table 2) refers to a small commercial turbocharger rotor whose theoretical model was slightly adapted to run on FABs instead of floating ring bearings $[30,6]$. The total number of rotor component modes is $H=8$ modes, comprising 4 modes in each of the $x z$ and $y z$ planes, as shown in Figure 25, where the first two modes are the $0 \mathrm{~Hz}$ modes $\varphi_{\text {trans }}(z)$ and $\varphi_{\text {rot }}(z)$ of eqs. $(55 \mathrm{a}, \mathrm{b})$. For the two identical FABs, $R_{\mathrm{L}}=10 \times 10^{-3} \mathrm{~m} ; L_{\mathrm{L}}=20 \times 10^{-3} \mathrm{~m} ; c_{\mathrm{L}}=1.6 \times 10^{-6} \mathrm{~m}$, viscosity $\mu=1.95 \times 10^{-5} \mathrm{~Pa} \cdot \mathrm{s}$; $p_{\mathrm{a}}=101325 \mathrm{~Pa}$. For the SEFM, the damping loss factor is 0.25 and the bump foil stiffness is 23.6950 $\mathrm{GN} / \mathrm{m}^{3}$. All these parameters were used in [30]. The relatively high stiffness value is justified for a bearing of this size - in fact, for a similar size bearing in Table 1 of reference [24], the stiffness was calculated to be $43.223 \mathrm{GN} / \mathrm{m}^{3}$ using SEFM formula in eq. (15) of reference [15]. The radial clearance is rather low, but was chosen in [30] since it gave (linear) stability beyond $88 \mathrm{krpm}$. 


\section{Figure 25}

Figure 26 shows the unfiltered eigenfrequency vs speed map, and Figure 27 shows the Campbell diagram extracted from this using the minimum journal amplitude criterion, eq. (51), with $C=0.1$. The Campbell diagram is of the typical form for a high-speed flexible rotor, with modes appearing in forward/reverse whirl pairs (modes 1, 2; modes 3, 4; modes 5, 6; modes 7,8) that separate out with increasing speed due to the gyroscopic effect [7]. Additionally, there are multiple half-frequency (0.5 EO) whirl modes that are responsible for the instability below $88 \mathrm{krpm}$.

\section{Figure 26}

\section{Figure 27}

Figure 28 shows the unstable $0.5 \mathrm{EO}$ whirl mode at $72 \mathrm{krpm}$. When the TNDA is performed at zero unbalance with initial conditions specific to this mode, the trajectory diverges in accordance with the predicted instability but eventually settles down to a high amplitude oscillation (limit cycle). The vibrating form (operating deflection shape (ODS)) and frequency of this limit cycle were presented in [30] and the present Campbell diagram analysis reveals them to be similar to stable mode 2 (Figure 29(a)), which has a conical form and is the lowest mode in forward whirl. Figure 29(b) shows the next stable mode in forward whirl at $72 \mathrm{krpm}$, which has a "horn" shape. The use of TNDA with different unbalance distributions in [30] revealed the occurrence of unbalance-driven non-synchronous vibration in the region of (linear) stability (as also observed in $[10,11]$ and Figures $6(a, b)$ ). The ODS of the nonlinear unbalance response simulations presented in [30], as well as their non-sychrononous frequency component, can now be associated with either mode 2 or mode 4 in Figures 29(a,b).

\section{Figure 28}

\section{Figure 29}


The nonlinear simulations in [30] at $2500 \mathrm{rev} / \mathrm{s}$ and $3000 \mathrm{rev} / \mathrm{s}$ showed that, for a particular unbalance distribution, the ODS exhibited purely synchronous bending vibration, thus indicating the proximity of the first bending critical speed. This can now be related to the intersection of the 1 EO line with mode 6 (forward whirl) at $2.9 \mathrm{kHz}$ in Figure 27, for which the mode shape is presented in Figure 30(a). It is noted from Figure 27 that the 1 EO line intersects with mode 7 at $2.5 \mathrm{kHz}$ but this reverse whirl mode was definitely not excited by the unbalance in [30] since its vibrating shape (see Figure 30(b)) does not match with the ODS at $150 \mathrm{krpm}(2.5 \mathrm{kHz})$ presented in [30]. This latter ODS, and the one at $180 \mathrm{krpm}$ (3 kHz) [30], in fact match mode 6 in Figure 30(a). It should also be noted that the occurrence of the first bending critical was erroneously explained in [30] with reference to the free-free modes (Figure 25) since the author did not, at that time, possess the Campbell diagram in Figure 27 for guidance.

\section{Figure 30}

\section{CONCLUSIONS}

This paper has established, for the first time, a methodology for the generation of the Campbell diagrams of a generic FAB-rotor system via direct extraction from its dynamical system model, thus avoiding Lund's FC method. This work was motivated by reported discrepancies between transient nonlinear dynamic analysis (TNDA) and the FC method for free low-amplitude dynamic analysis. The new method was based on the eigenvalue analysis of the state Jacobian of the recently established dynamical system representation of a generic rotor-FAB system. Through appropriate scaling of the eigenvectors and the application of a minimum journal amplitude criterion, it was found that the multitude of eigenvalues/eigenvectors of the Jacobian could be filtered in order to extract only the relevant modes. Where appropriate, a maximum damping criterion was also found necessary. Each extracted mode could be precisely verified for its frequency, damping ratio (stability) and whirl direction using TNDA of the dynamical system through mode-specific low-amplitude initial conditions. Such verification was not 
previously presented for FC-based Campbell diagrams. The methodology was successfully tested on three cases in the literature, which respectively illustrated its applicability to complex bearing types (3pad), complex foil models (FFSMM), and flexible rotors. The main case was one for which significant discrepancies between FC and TNDA stability predictions had been previously reported in the literature. The replacement of the FC method by the present linearised method was shown to completely eliminate such discrepancies. This contribution lays the groundwork for a potential future study that will benchmark traditional FC-based Campbell diagrams against those produced by this paper's novel method. This research completes the framework of the computational suite in Figure 1. Future research will focus on developing more advanced foil and air film models which will continue to be cast in the state space form of eq. (1) to benefit from the present methodology.

\section{ACKNOWLEDGMENTS}

The author acknowledges the support of the Engineering and Physical Sciences Research Council of the UK who funded the initial development of the computational suite in Figure 1 through EP/I029184/1. Datasets for sections 4-6 have been provided.

\section{DECLARATIONS OF INTEREST}

None.

\section{REFERENCES}

[1] P. Bonello, M.F. Bin Hassan, An experimental and theoretical analysis of a foil-air bearing rotor system, Journal of Sound and Vibration 413 (2018) 395-420, doi: 10.1016/j.jsv.2017.10.036.

[2] E. Ott, Chaos in Dynamical Systems, Cambridge University Press, Cambridge, 1993.

[3] L. F. Shampine, M. W. Reichelt, The Matlab ODE suite, SIAM J. Sci. Comput. 18 (1) (1997) 1-22.

[4] R. Seydel, From Equilibrium to Chaos: Practical Bifurcation and Stability Analysis, Elsevier Science, New York, 1988. 
[5] P. Bonello, H. M. Pham, The efficient computation of the nonlinear dynamic response of a foil-air bearing rotor system, Journal of Sound and Vibration 333 (15) (2014) 3459-3478, doi: 10.1016/j.jsv.2014.03.001.

[6] P. Bonello, H. M. Pham, Nonlinear dynamic analysis of high speed oil-free turbomachinery with focus on stability and self-excited vibration, Journal of Tribology 136(4) (2014) 041705, doi:10.1115/1.4027859.

[7] E. Kramer, Dynamics of Rotors and Foundations, Springer-Verlag, Berlin, 1993.

[8] D. Kim, Parametric studies on static and dynamic performance of air foil bearings with different top foil geometries and bump stiffness distributions, Journal of Tribology 129 (2) (2007) 354-364, doi:10.1115/1.2540065.

[9] R. Hoffmann, T. Pronobis, R. Liebich, Non-linear stability analysis of a modified gas foil bearing structure, in: Proceedings of the 9th IFToMM International Conference on Rotor Dynamics, 2015, pp. 1259-1276, http://dx.doi.org/10.1007/978-3-319-06590-8_103.

[10] J.S. Larsen, B.B. Nielsen, I.F. Santos, On the Numerical Simulation of Nonlinear Transient Behaviour of Compliant Air-Foil Bearings, in: Proceedings of SIRM 2015, Magdeburg, Deutschland, 2015, Paper-ID 39.

[11] J.S. Larsen, I.F. Santos, On the nonlinear steady-state response of rigid rotors supported by air foil bearings - Theory and experiments, Journal of Sound and Vibration 346 (2015) 284-297, doi:10.1016/j.jsv.2015.02.017.

[12] Z. Guo, K. Feng, T. Liu, P. Lyu, T. Zhang, Nonlinear dynamic analysis of rigid rotor supported by gas foil bearings: Effects of gas film and foil structure on subsynchronous vibrations, Mechanical Systems and Signal Processing 107 (2018) 549-566, doi: 10.1016/j.ymssp.2018.02.005 . 
[13] H.M. Pham, P. Bonello, Efficient Techniques for the Computation of the Nonlinear Dynamics of a Foil-Air Bearing-Rotor System, in: Proceedings of the ASME Turbo Expo 2013, San Antonio, Texas, USA, paper no. GT-2013-94389.

[14] J.S. Larsen, I.F. Santos, S. von Osmanski, Stability of rigid rotors supported by air foil bearings: Comparison of two fundamental approaches, Journal of Sound and Vibration 381 (2016) 179-191, doi:10.1016/j.jsv.2016.06.022

[15] B.B. Nielsen, I.F. Santos, Transient and steady state behaviour of elasto-aerodynamic air foil bearings, considering bump foil compliance and top foil inertia and flexibility: a numerical investigation, Proc. IMechE Part J: Journal of Engineering Tribology (2017), doi: $10.1177 / 1350650117689985$.

[16] T. Leister, C. Baum, W. Seemann, On the importance of frictional energy dissipation in the prevention of undesirable self-excited vibrations in gas foil bearing rotor systems, Technische Mechanik 37, 2-5, (2017), 280-290, doi: 0.24352/UB.OVGU-2017-104.

[17] J.W. Lund, Calculation of stiffness and damping properties of gas bearings, Journal of Lubrication Technology (1968) 793-804.

[18] J.P. Peng, M. Carpino, Calculation of stiffness and damping coefficients for elastically supported gas foil bearings, Journal of Tribology 115 (1993) 20-27.

[19] R. Hoffmann, R. Liebich, Characterisation and calculation of nonlinear vibrations in gas foil bearing systems - An experimental and numerical investigation, Journal of Sound and Vibration 412 (2018) 389-409, doi: 10.1016/j.jsv.2017.09.040.

[20] T.H. Kim, L. San Andrés, Analysis of advanced gas foil bearings with piecewise linear elastic supports, Tribology International 40 (2007) 1239-1245. 
[21] P. Vleugels, T. Waumans, J. Peirs, F. Al-Bender, D. Reynaerts, High speed bearings for micro gas turbines: stability analysis of foil bearings, Journal of Micromechanics and Microengineering 16 (2006) 282-289.

[22] Z. Guo, L. Peng, K. Feng, W. Liu, Measurement and prediction of nonlinear dynamics of a gas foil bearing supported rigid rotor system, Measurement 121 (2018) 205-217, doi: 10.1016/j.measurement.2017.12.039.

[23] K. Sim, Y-B Lee, T. H. Kim, J. Lee, Rotordynamic performance of shimmed gas foil bearings for oil-free turbochargers, Journal of Tribology 134 (2012) 031102, doi: 10.1115/1.4005892.

[24] K. Sim, Y-B Lee, T. H. Kim, Effects of mechanical preload and bearing clearance on rotordynamic performance of lobed gas foil bearings for oil-free turbochargers, Tribology Transactions 56 (2013) 224-235, doi: 10.1080/10402004.2012.737502.

[25] J.S. Larsen, Nonlinear Analysis of Rotors Supported by Air Foil Journal Bearings - Theory and Experiments, Ph.D. thesis, DTU Mechanical Engineering, DCAMM Special Report, no. S177, ISBN 978-87-7475-402-2, Technical University of Denmark, Lingby, Denmark, 2014.

[26] M.F. Bin Hassan, P. Bonello, A neural network identification technique for a foil-air bearing under variable speed conditions and its application to unbalance response analysis, Journal of Tribology, 139(2) (2017) 021501, doi:10.1115/1.4033455.

[27] W. Campbell, The protection of steam turbine disk wheels from axial vibration, Transactions of the ASME 46 (1924) 31-160.

[28] J. Simek, Specific features of aerodynamic journal bearings with elastically supported pads, Lubricants 5, 10 (2017) 255-273, doi: 10.1016/j.jsv.2017.02.028.

[29] M.F. Bin Hassan, P. Bonello, A new modal-based approach for modelling the bump foil structure in the simultaneous solution of foil-air bearing rotor dynamic problems, Journal of Sound and Vibration 396 (2017) 255-273, doi: 10.3390/lubricants5020010. 
[30] P. Bonello, Simulations of the nonlinear dynamic response of a turbocharger on foil-air bearings with focus on unbalance excitation, in: VIRM 11 - Vibrations in Rotating Machinery, Manchester UK 13-16 September 2016, Institution of Mechanical Engineers, ISBN (Print) 978-0-9572374-8-3.

[31] P. Bonello, A new method for the calculation of the Campbell diagram of a foil-air bearing rotor model, in: SIRM $2019-13^{\text {th }}$ International Conference on Dynamics of Rotating Machines, Copenhagen, Denmark, 2019, Paper ID- SIRM2019-41.

[32] M.L. Adams, Non-linear dynamics of flexible multi-bearing rotors, Journal of Sound and Vibration 71 (1) (1980) 129-144.

[33] P. Bonello, P., Transient Modal Analysis of the Nonlinear Dynamics of a Turbocharger on Floating Ring Bearings, Proc. IMechE Part J: Journal of Engineering Tribology 223 (2009) 79-93.

[34] B. B. Nielsen, Combining Gas Bearing and Smart Material Technologies for Improved Machine Performance: Theory and Experiment, Ph.D. thesis, DTU Mechanical Engineering. DCAMM Special Report, no. S221, ISBN 978-87-7475-481-7 Technical University of Denmark, Lingby, Denmark, 2017.

[35] G. Dahlquist, Numerical Methods, Prentice Hall, Englewood Cliffs N. J., 1974.

[36] Symbolic Math Toolbox ${ }^{\mathrm{TM}}$ - User's Guide, The MathWorks, Inc, Natick MA, 2018.

[37] S. S. Rao, Mechanical Vibrations (Fifth Edition), Prentice Hall, Upper Saddle River N. J., 2011. 


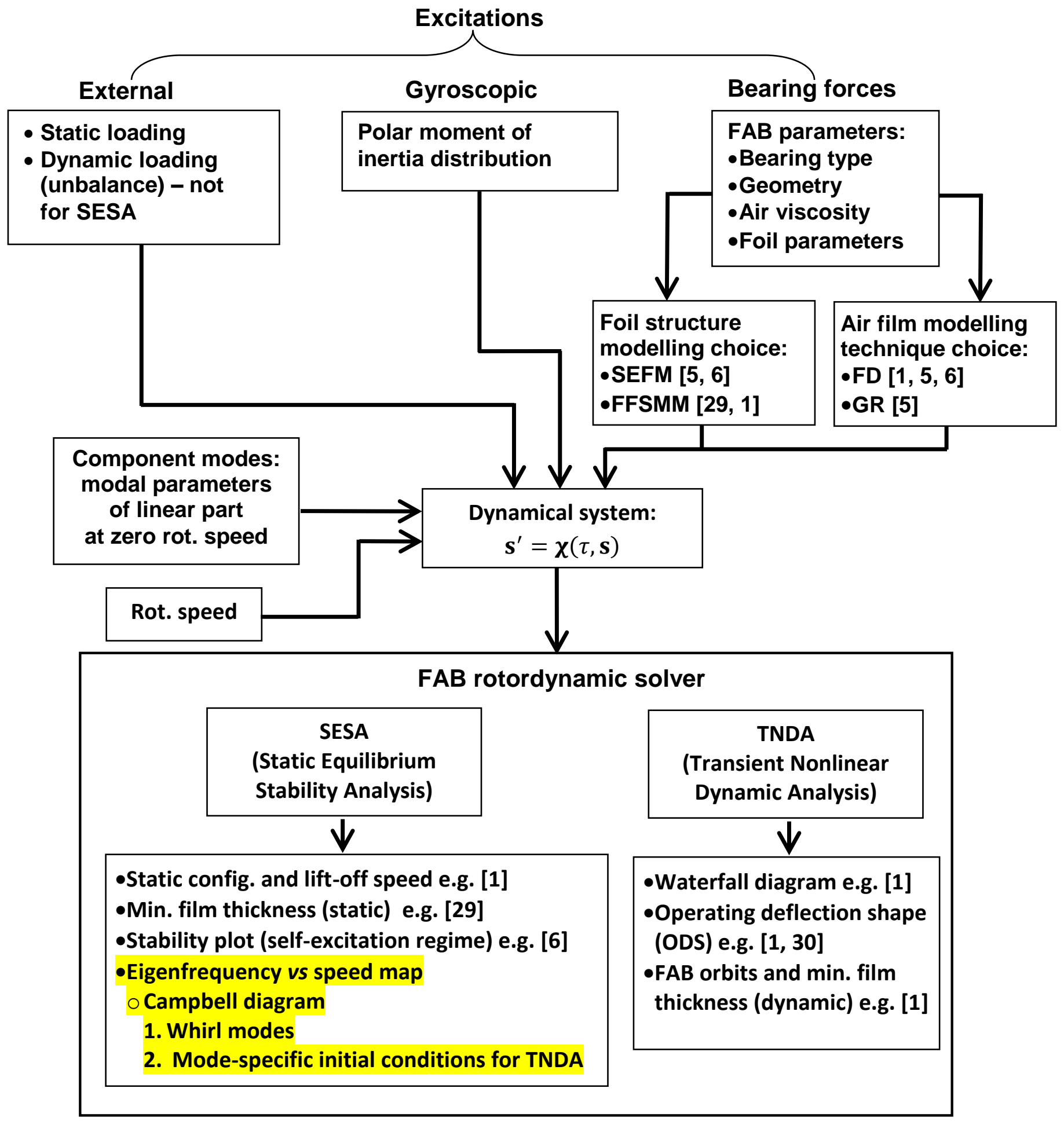

Figure 1. Overview of the computational suite developed at the University of Manchester for the simulation of the dynamic performance of rotating machinery running on foil-air bearings (FABs) - the novel addition presented in this paper is highlighted (SEFM - simple equivalent foundation model; FFSMM - full foil structure modal model; FD - finite difference; GR - Galerkin Reduction). 

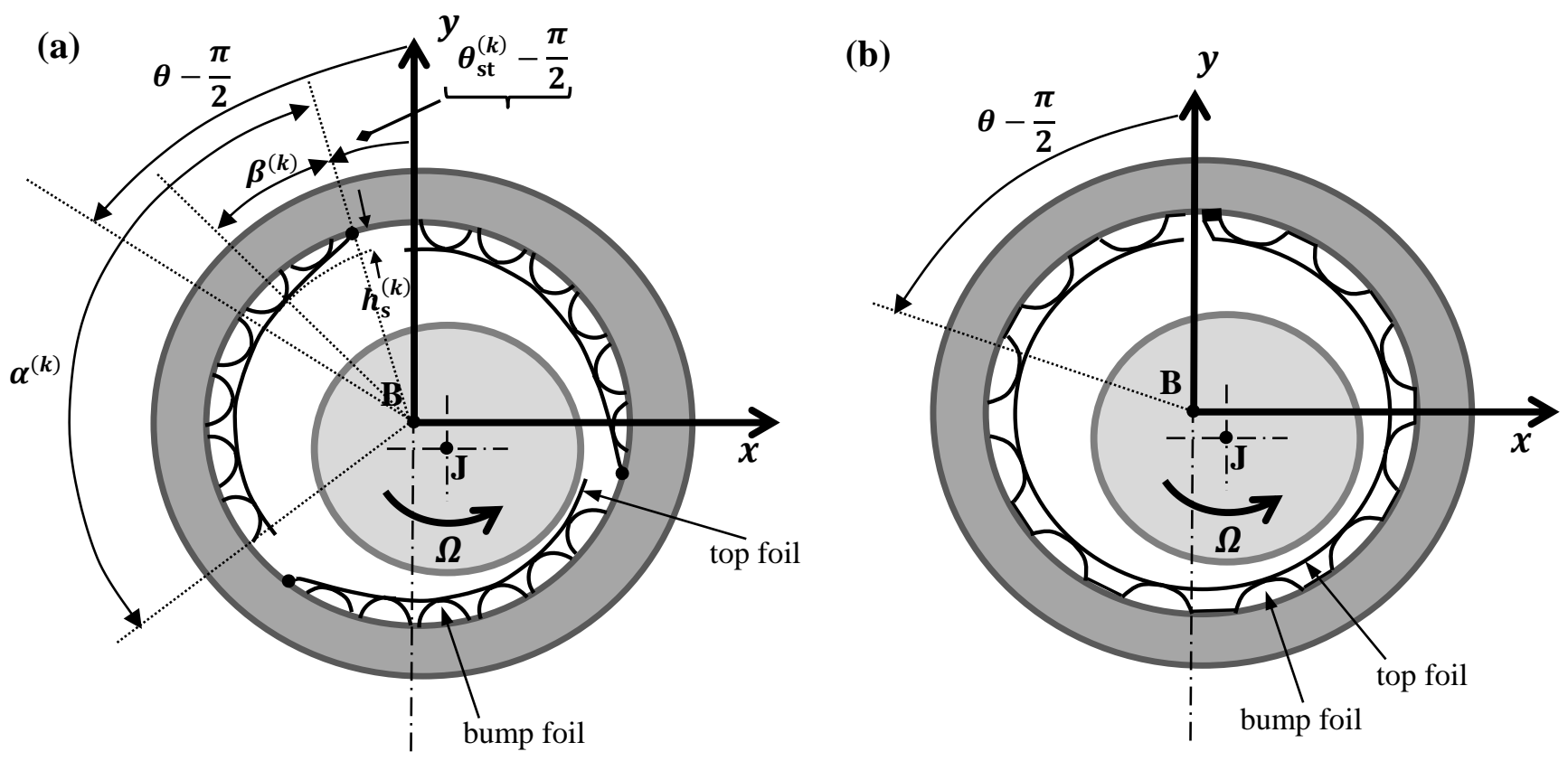

Figure 2. Foil-air bearing types considered in this paper: (a) three-pad bearing (Case 1, Table 2); (b) single-pad bearing (Cases 2, 3, Table 2).

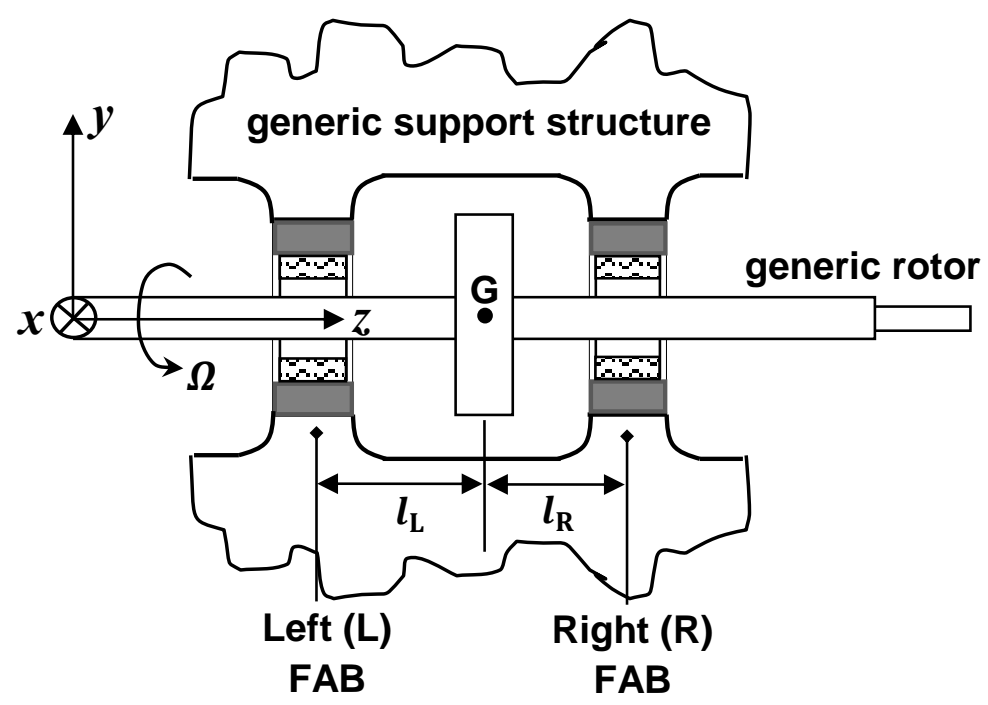

Figure 3. Generic representation of a rotating machine running on two FABs. 
(a)

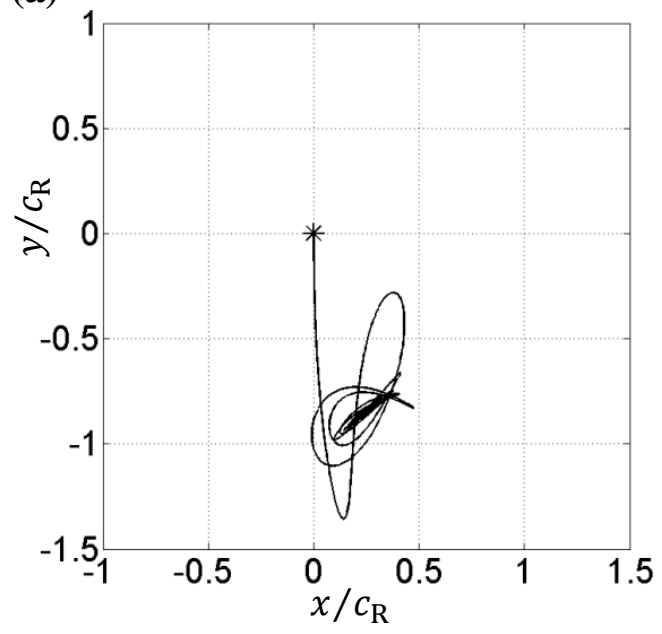

(c)

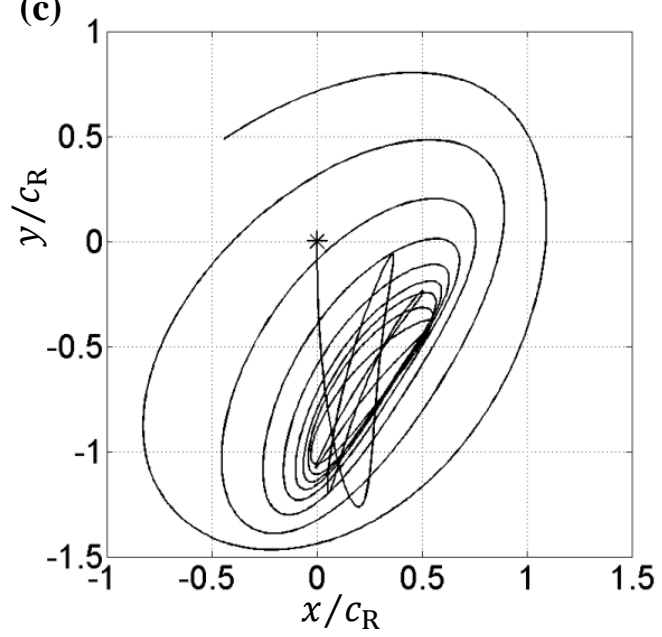

(b)

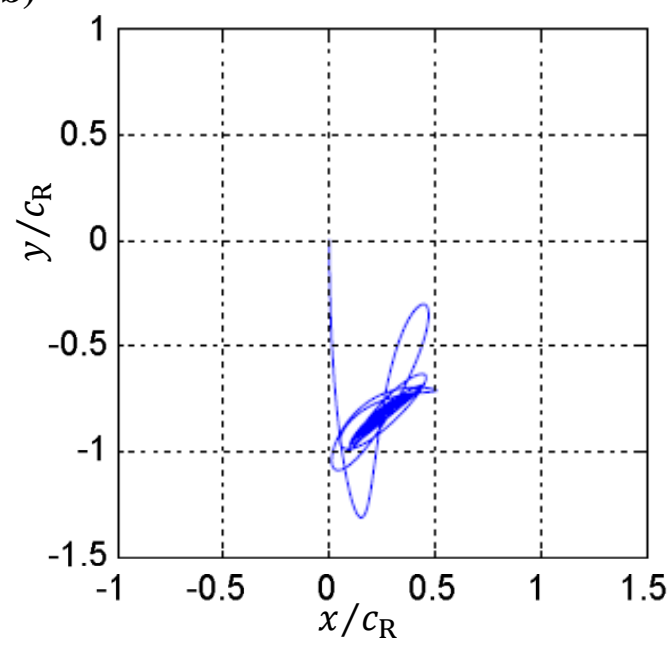

(d)

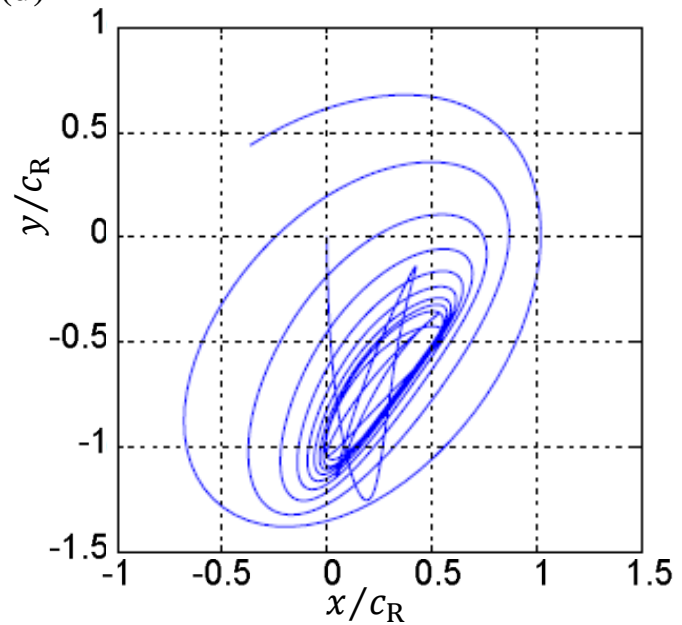

Figure 4. TNDA simulation of trajectory of right (R) FAB journal centre from default initial conditions at zero rotor unbalance for Case no.1 (Table 2) with $K_{\mathrm{b}_{\mathrm{L}}}=K_{\mathrm{b}_{\mathrm{R}}}=9.26 \times 10^{9} \mathrm{~N} / \mathrm{m}^{3}:$ (a) 20 krpm, present model; (b) $20 \mathrm{krpm}$, Larsen and Santos result [10]; (c) $30 \mathrm{krpm}$, present model (7×49 FD grid per pad); (d) $30 \mathrm{krpm}$, Larsen and Santos result [10]. (NB: "7×49 FD grid per pad" is equivalent to a $15 \times 51$ grid covering entire pad considering symmetry and edges). 

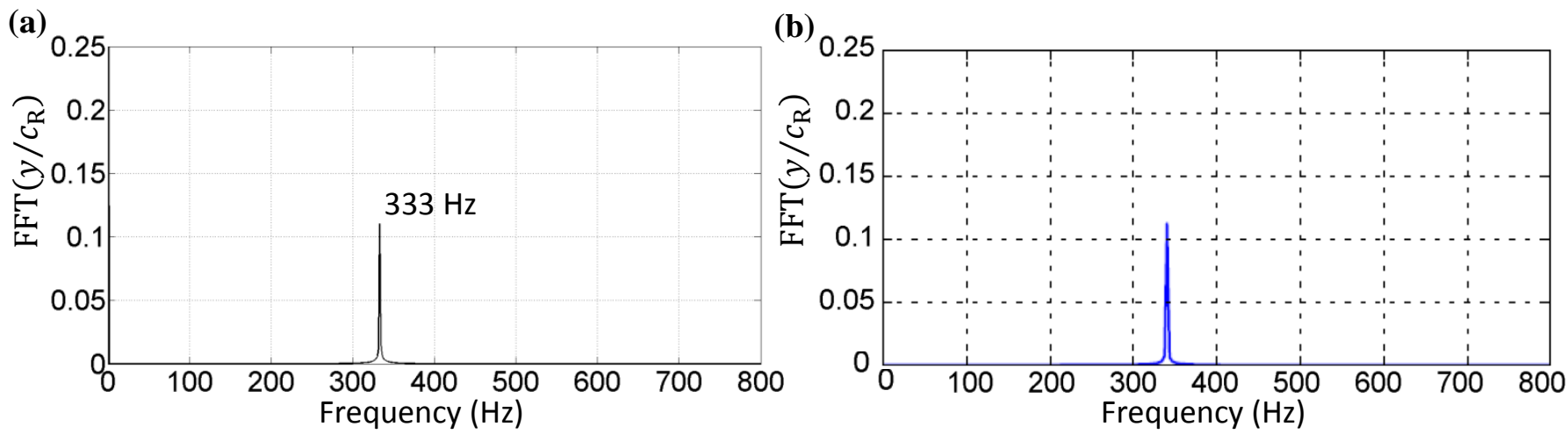

Figure 5. Frequency spectrum of steady-state $y$ vibration response of the right (R) FAB journal at 20 $\mathrm{krpm}$ for Case no.1 (Table 2) with $K_{\mathrm{b}_{\mathrm{L}}}=K_{\mathrm{b}_{\mathrm{R}}}=9.26 \times 10^{9} \mathrm{~N} / \mathrm{m}^{3}$ and equivalent unbalance of 20 g.mm applied at each FAB, $180^{\circ}$ out of phase: (a) present model ( $7 \times 49$ FD grid per pad); (b) Larsen and Santos result [10].

(a)

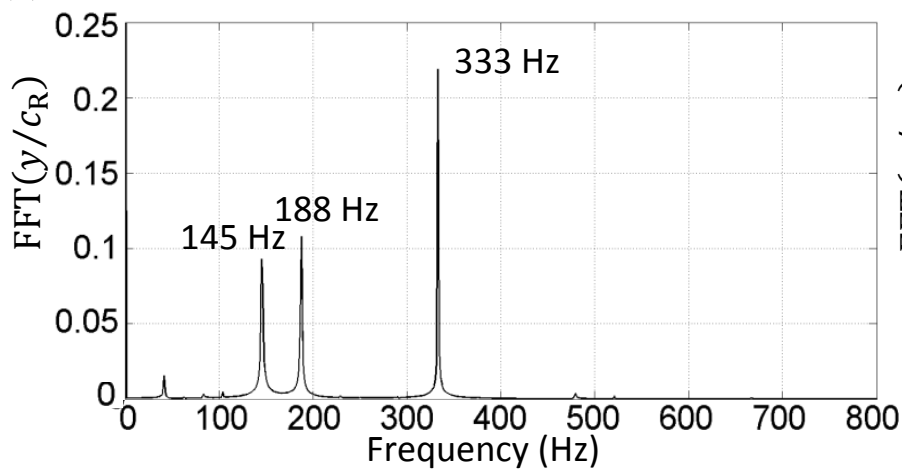

(b)

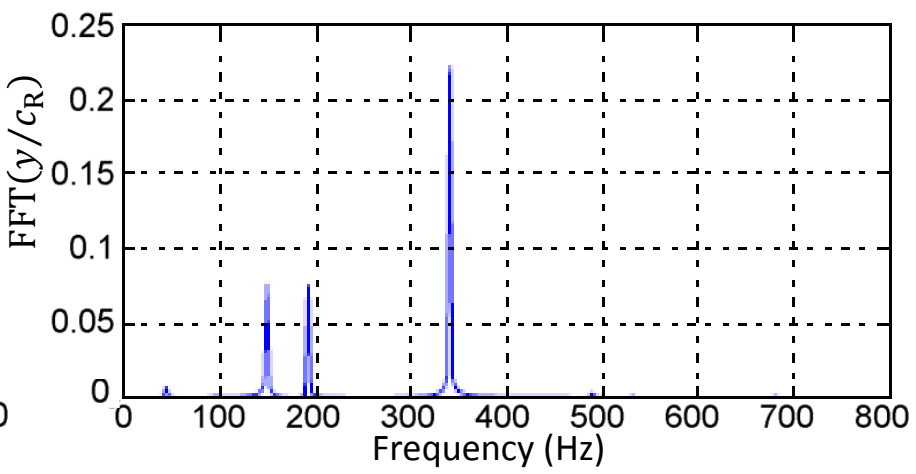

Figure 6. Frequency spectrum of steady-state $y$ vibration response of the right (R) FAB journal at 20 $\mathrm{krpm}$ for Case no.1 (Table 2) with $K_{\mathrm{b}_{\mathrm{L}}}=K_{\mathrm{b}_{\mathrm{R}}}=9.26 \times 10^{9} \mathrm{~N} / \mathrm{m}^{3}$ and equivalent unbalance of $40 \mathrm{~g} \cdot \mathrm{mm}$ applied at each FAB, $180^{\circ}$ out of phase: (a) present model ( $7 \times 49$ FD grid per pad); (b) Larsen and Santos result [10]. 

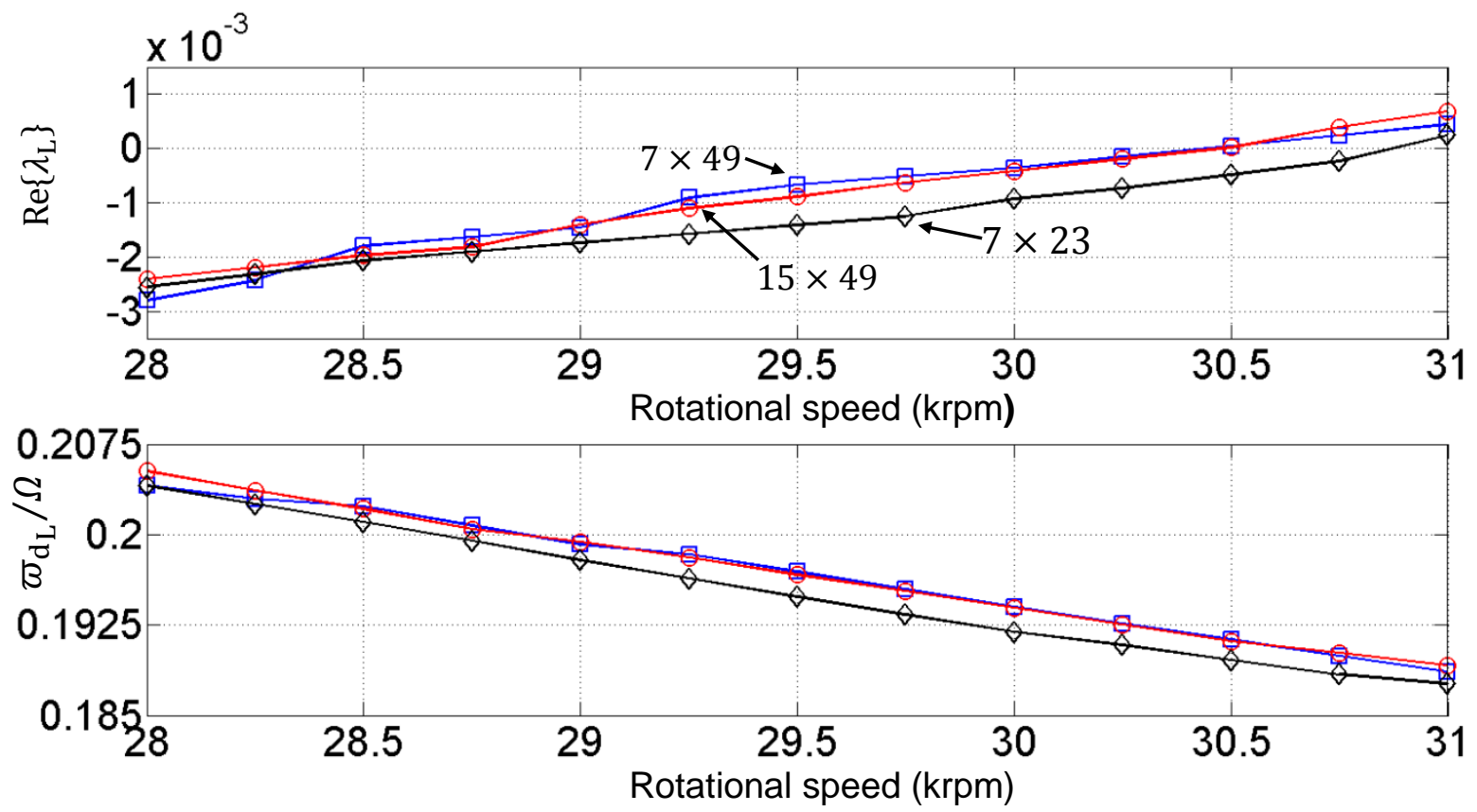

Figure 7. $\operatorname{Re}\left\{\lambda_{\mathrm{L}}\right\}$ vs rotational speed (upper figure), $\varpi_{\mathrm{d}_{\mathrm{L}}} / \Omega\left(=\left|\operatorname{Im}\left\{\lambda_{\mathrm{L}}\right\}\right| / 2\right)$ vs rotational speed (lower figure) for Case no. 1 (Table 2) with $K_{\mathrm{b}_{\mathrm{L}}}=K_{\mathrm{b}_{\mathrm{R}}}=8.8 \times 10^{9} \mathrm{~N} / \mathrm{m}^{3}$ and different FD grid sizes $N_{z} \times N_{\theta}$ per pad: $7 \times 23$ (black diamonds); $7 \times 49$ (blue squares); $15 \times 49$ (red circles).
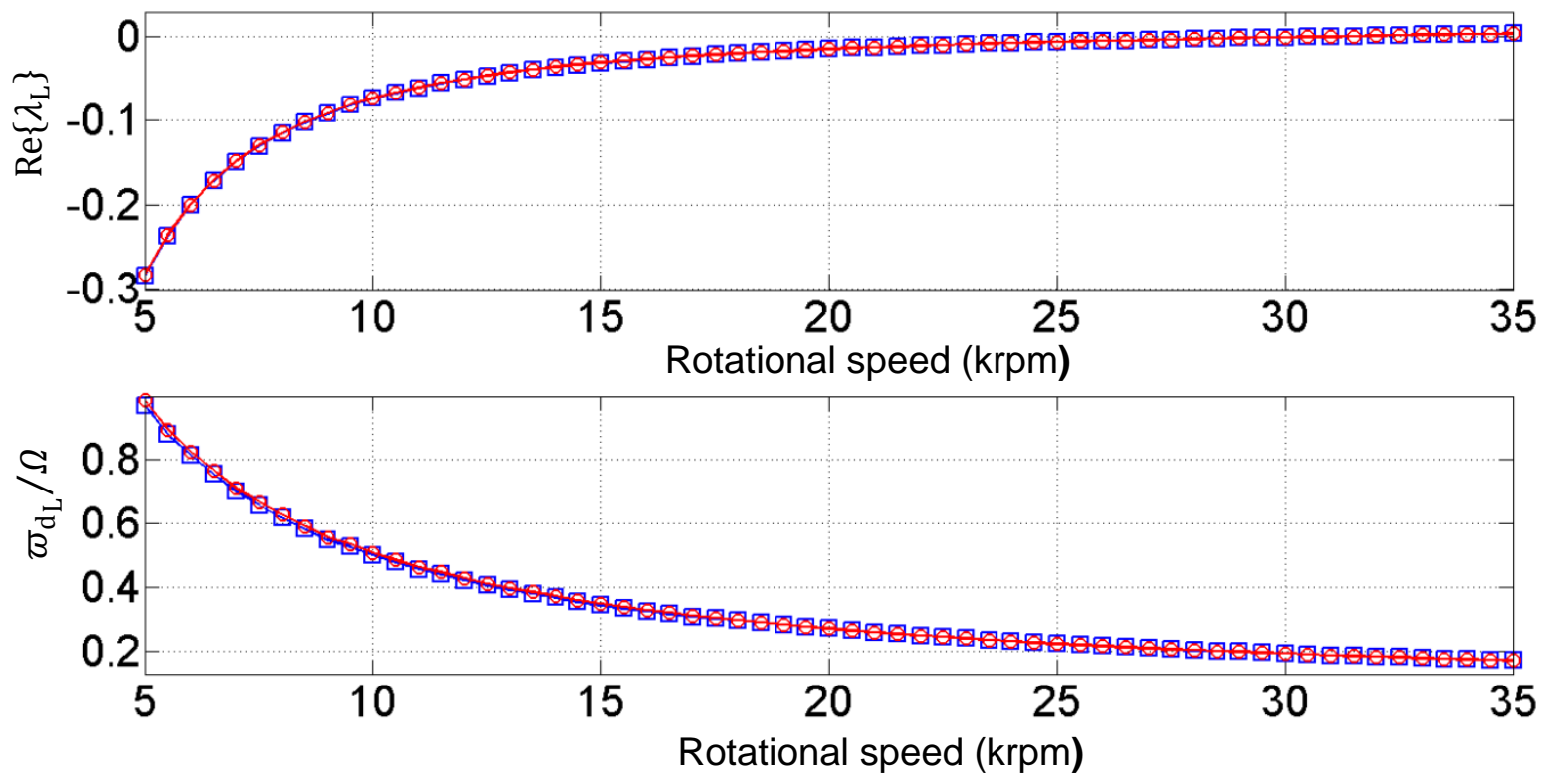

Figure 8. $\operatorname{Re}\left\{\lambda_{\mathrm{L}}\right\}$ vs rotational speed (upper figure), $\varpi_{\mathrm{d}_{\mathrm{L}}} / \Omega\left(=\left|\operatorname{Im}\left\{\lambda_{\mathrm{L}}\right\}\right| / 2\right)$ vs rotational speed (lower figure) for Case no. 1 (Table 2) with $K_{\mathrm{b}_{\mathrm{L}}}=K_{\mathrm{b}_{\mathrm{R}}}=8.8 \times 10^{9} \mathrm{~N} / \mathrm{m}^{3}, 7 \times 49$ FD grid per pad and Jacobian computed by two different methods: fully numerical method using numjac.m [3] (red circles); part-analytical method of section 3.1.1 (blue squares). 


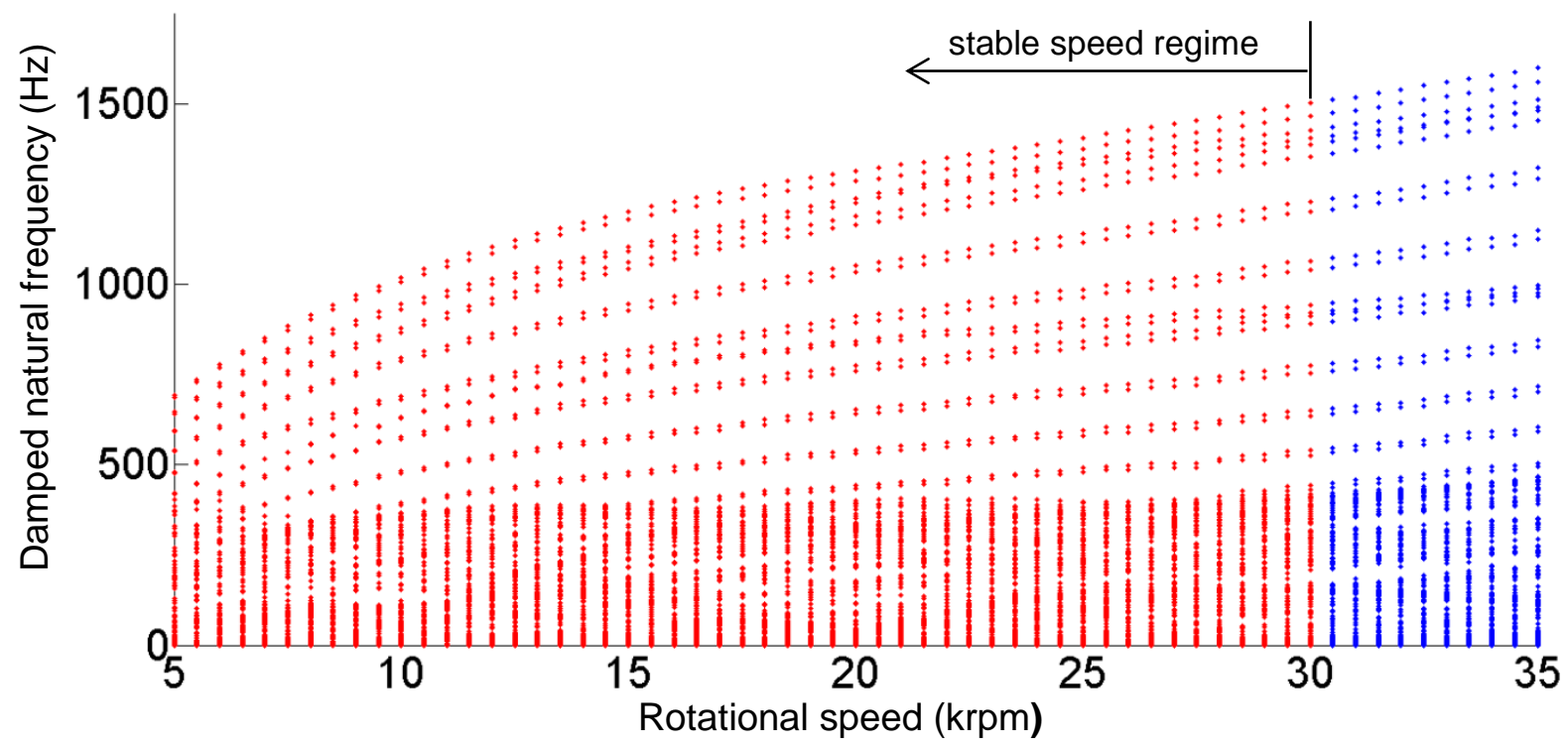

Figure 9. Unfiltered eigenfrequency $\varpi_{\mathrm{d}, n} /(2 \pi) v s$ speed map for Case no. 1 (Table 2) with $K_{\mathrm{b}_{\mathrm{L}}}=K_{\mathrm{b}_{\mathrm{R}}}=8.8 \times 10^{9} \mathrm{~N} / \mathrm{m}^{3}$ and $7 \times 49$ FD grid per pad.

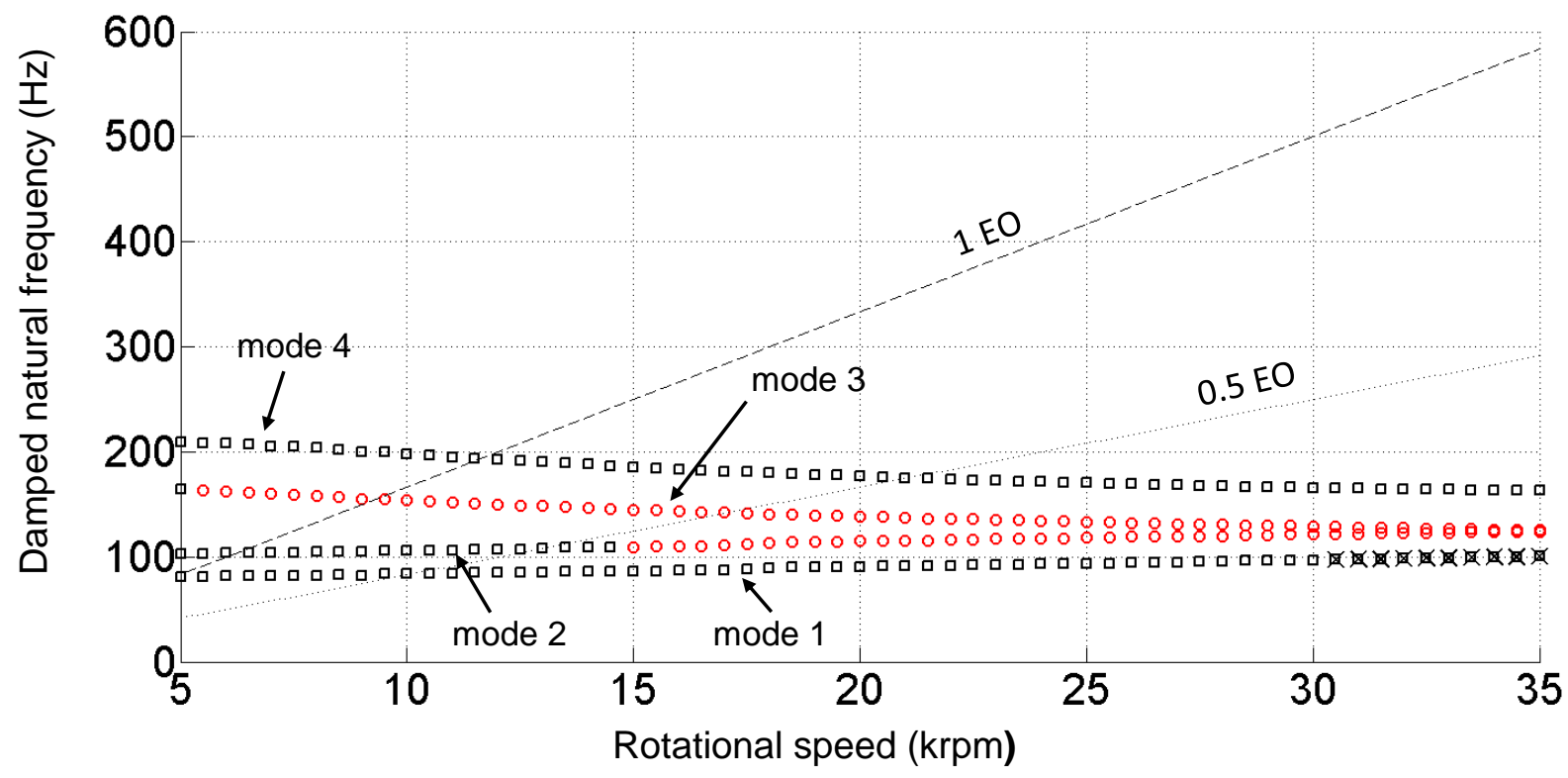

Figure 10. Campbell diagram for Case no. 1 (Table 2) with $K_{\mathrm{b}_{\mathrm{L}}}=K_{\mathrm{b}_{\mathrm{R}}}=8.8 \times 10^{9} \mathrm{~N} / \mathrm{m}^{3}$ and $7 \times 49$ FD grid per pad, extracted from raw eigenfrequency $v s$ speed map of Figure 9 using minimum journal amplitude criterion, eq. (51), with $C=0.1$ : forward whirl (black squares); reverse whirl (red circles); unstable mode points overlaid with a cross; EO (engine order). 
(a) 2

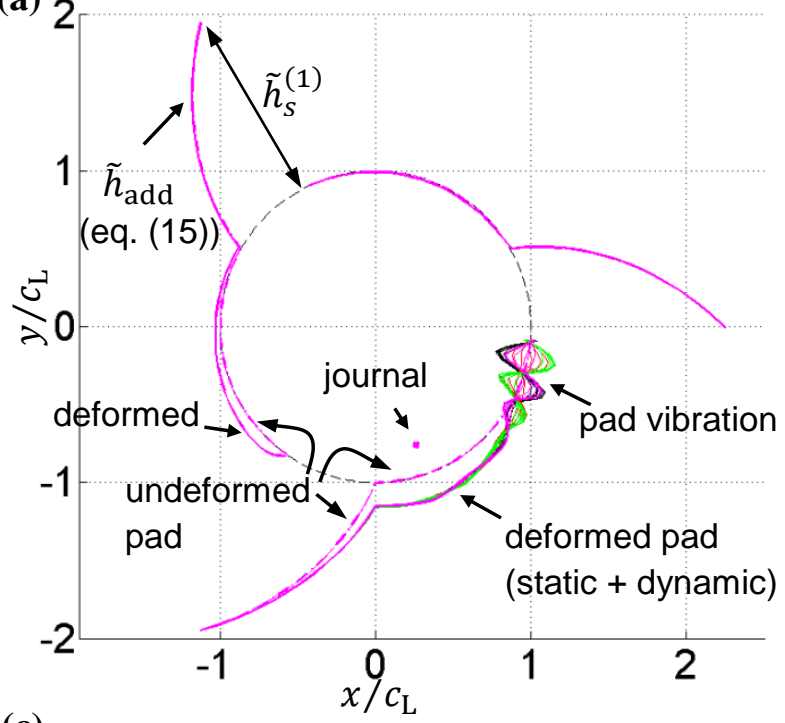

(c)

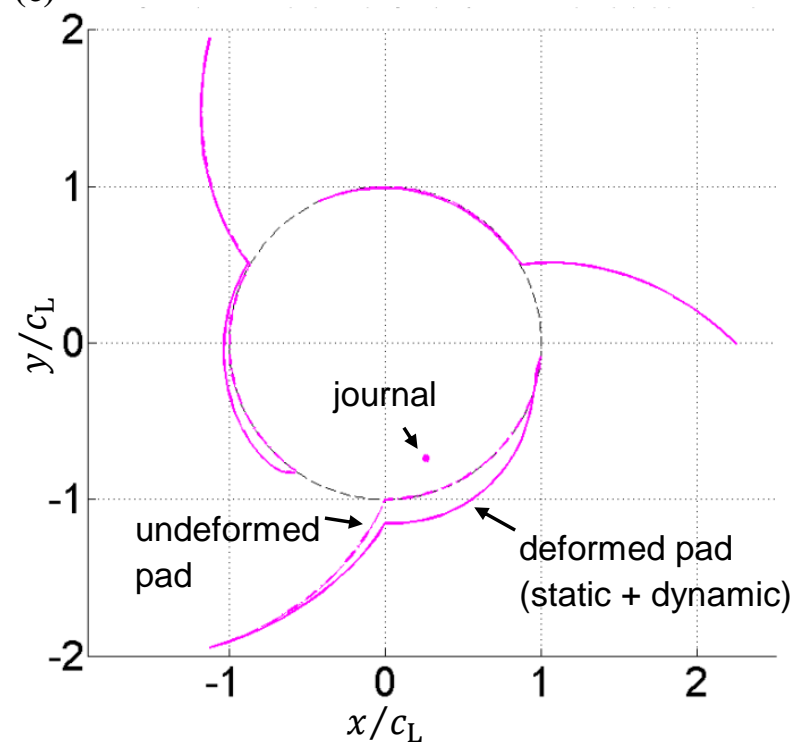

(b)

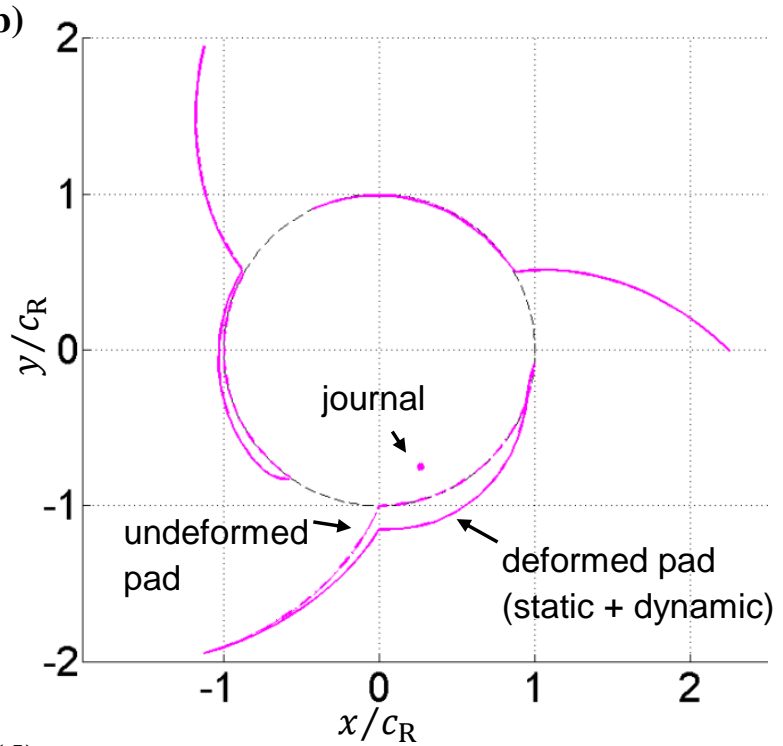

(d)

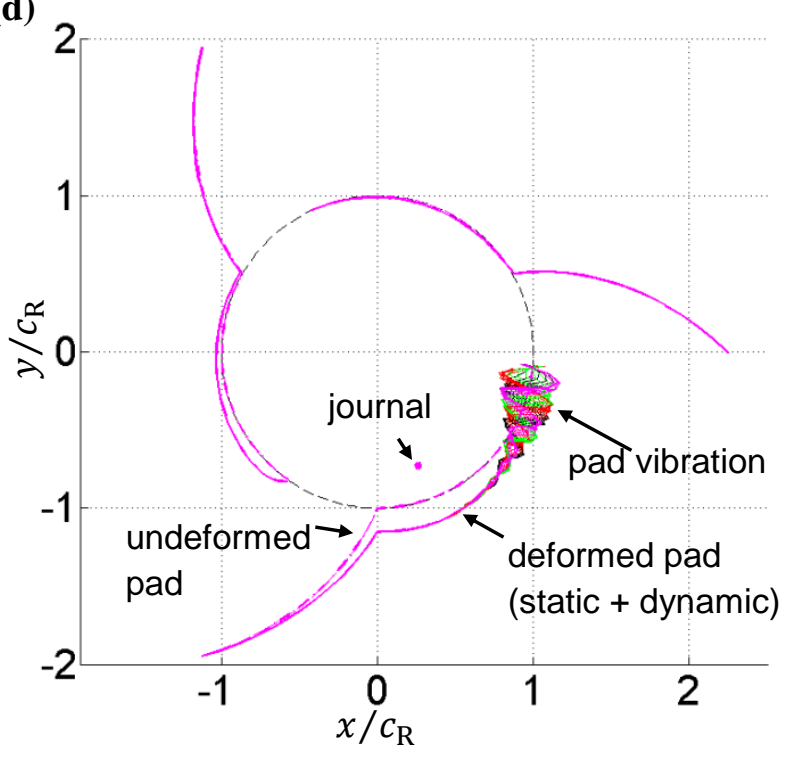

Figure 11. Two examples of modes rejected by minimum journal amplitude criterion, eq. (51), for Case no. 1 (Table 2) with $K_{\mathrm{b}_{\mathrm{L}}}=K_{\mathrm{b}_{\mathrm{R}}}=8.8 \times 10^{9} \mathrm{~N} / \mathrm{m}^{3}$ and $7 \times 49$ FD grid per pad: (a), (b) L, R FABs respectively at $29.5 \mathrm{krpm}$ with $f_{\mathrm{d}, n}=\varpi_{\mathrm{d}, n} /(2 \pi)=114.45 / \mathrm{Hz}, \zeta_{n}=0.99993$ (vibration only in one pad of L FAB); (c), (d) L, R FABs respectively at $32 \mathrm{krpm}$ with $f_{\mathrm{d}, n}=328.50 / \mathrm{Hz}, \zeta_{n}=$ 0.99982 (vibration only in one pad of R FAB) (NB: for clarity of display, the exponential decay factor $\mathrm{e}^{-\zeta_{n} \varpi_{\mathrm{u}, n} t}$ in eqs. (40), (53) was omitted when calculating the modal vibration). 
(a)

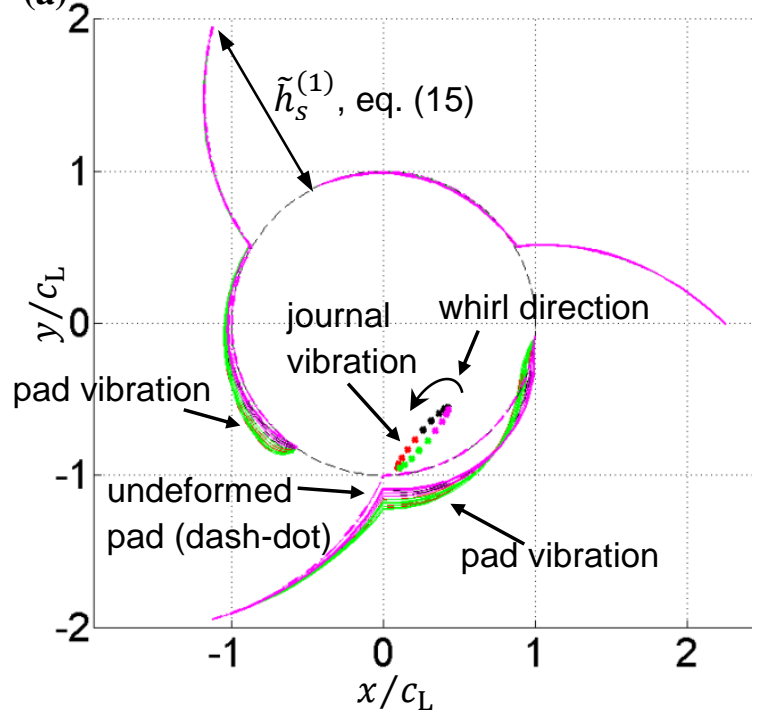

(b)

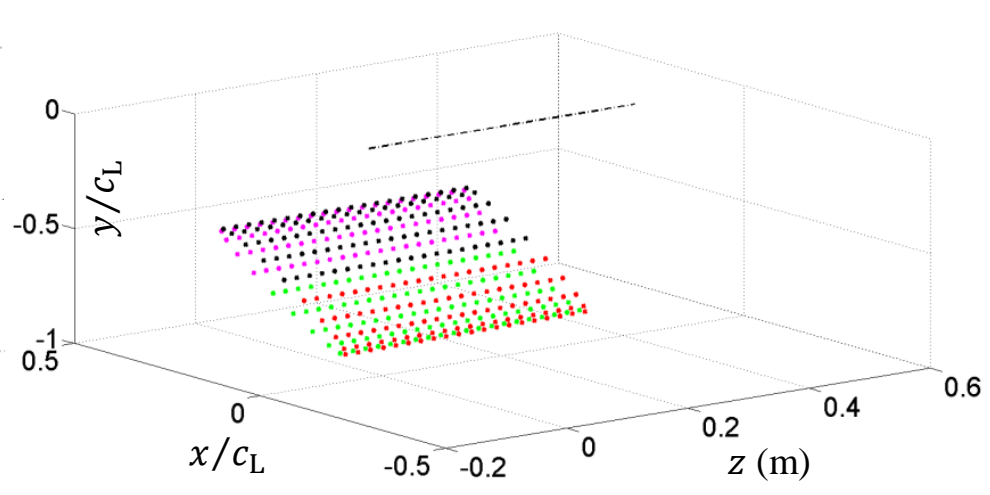

Figure 12. Mode 1 at $30 \mathrm{krpm}\left(f_{\mathrm{d}, n}=96.99 \mathrm{~Hz}, \zeta_{n}=0.00092\right.$, forward whirl $)$, taken from Campbell diagram of Figure 10 (Case no. 1, Table 2, with $K_{\mathrm{b}_{\mathrm{L}}}=K_{\mathrm{b}_{\mathrm{R}}}=8.8 \times 10^{9} \mathrm{~N} / \mathrm{m}^{3}$ and $7 \times 49$ FD grid per pad): (a) vibration at L FAB; (b) rotor mode shape. (NB for all figures in paper: temporal sequence of vibration in digital version of paper is indicated by colour sequence black-red-green-magenta; for clarity of display, the exponential decay factor $\mathrm{e}^{-\zeta_{n} \varpi_{\mathrm{u}, n} t}$ in eqs. (40), (53) was omitted when calculating the modal vibration).

(a)

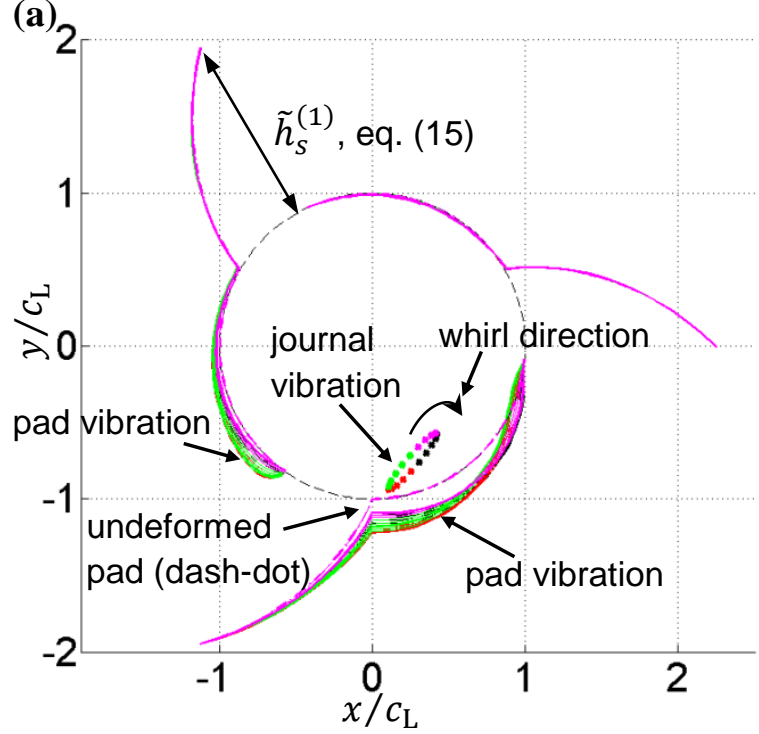

(b)

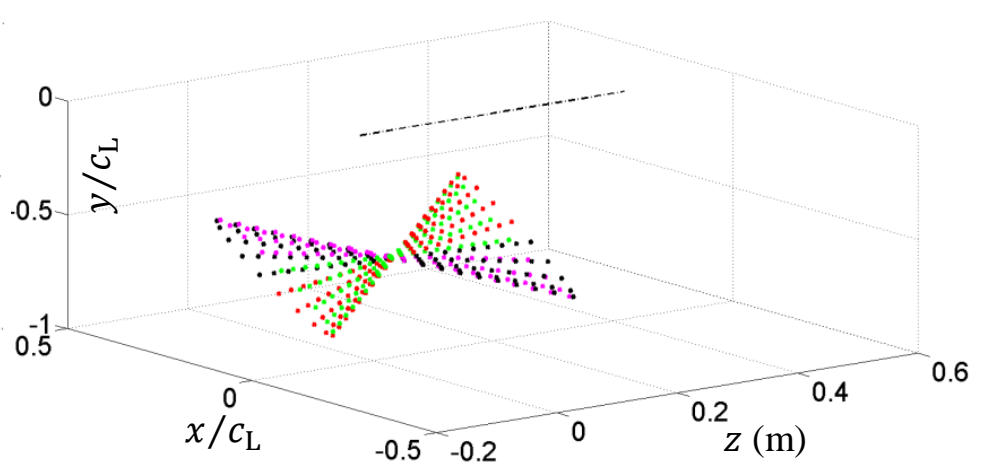

Figure 13. Mode 2 at $30 \mathrm{krpm}\left(f_{\mathrm{d}, n}=121.57 \mathrm{~Hz}, \zeta_{n}=0.080575\right.$, reverse whirl $)$, taken from Campbell diagram of Figure 10 (Case no. 1, Table 2, with $K_{\mathrm{b}_{\mathrm{L}}}=K_{\mathrm{b}_{\mathrm{R}}}=8.8 \times 10^{9} \mathrm{~N} / \mathrm{m}^{3}$ and $7 \times 49$ FD grid per pad): (a) vibration at L FAB; (b) rotor mode shape. 
(a)

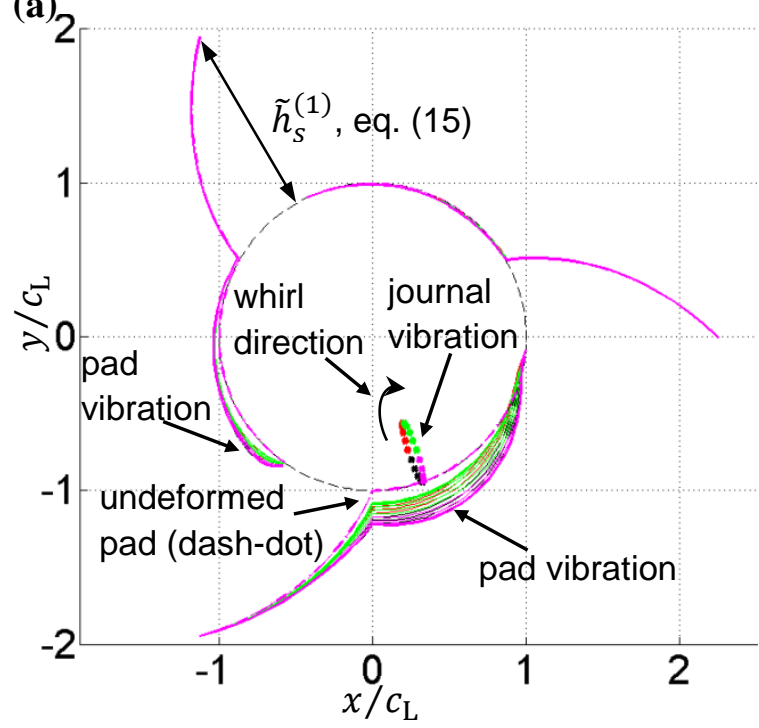

(b)

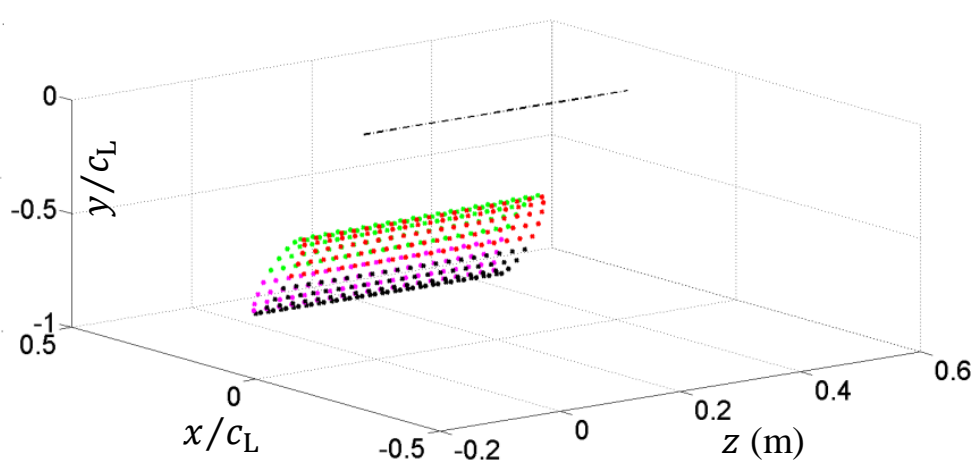

Figure 14. Mode 3 at $30 \mathrm{krpm}\left(f_{\mathrm{d}, n}=129.04 \mathrm{~Hz}, \zeta_{n}=0.080668\right.$, reverse whirl $)$, taken from Campbell diagram of Figure 10 (Case no. 1, Table 2, with $K_{\mathrm{b}_{\mathrm{L}}}=K_{\mathrm{b}_{\mathrm{R}}}=8.8 \times 10^{9} \mathrm{~N} / \mathrm{m}^{3}$ and $7 \times 49$ FD grid per pad): (a) vibration at L FAB; (b) rotor mode shape.

(a)

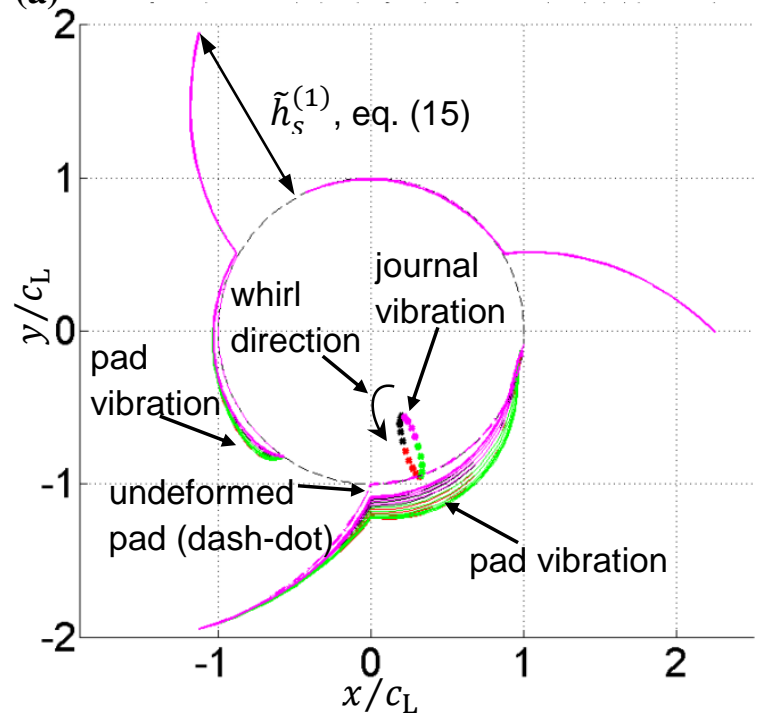

(b)

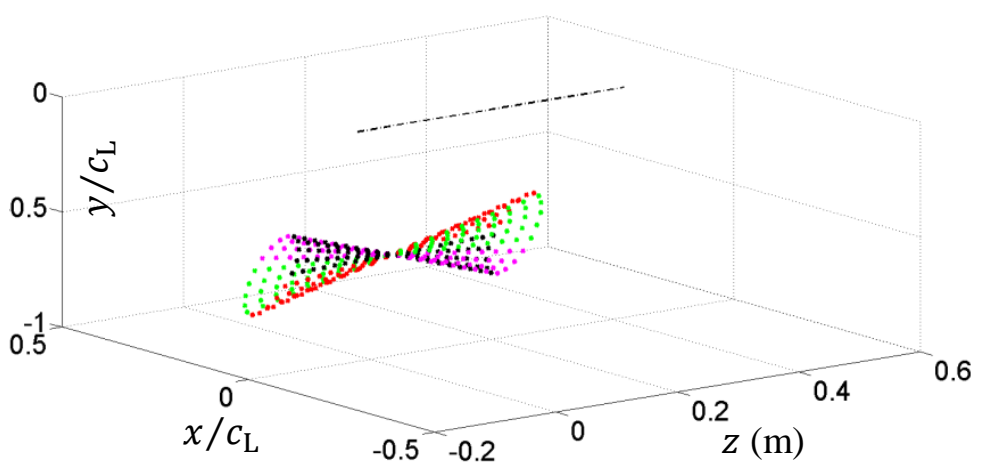

Figure 15. Mode 4 at $30 \mathrm{krpm}\left(f_{\mathrm{d}, n}=165.75 \mathrm{~Hz}, \zeta_{n}=0.04443\right.$, forward whirl $)$, taken from Campbell diagram of Figure 10 (Case no. 1, Table 2, with $K_{\mathrm{b}_{\mathrm{L}}}=K_{\mathrm{b}_{\mathrm{R}}}=8.8 \times 10^{9} \mathrm{~N} / \mathrm{m}^{3}$ and $7 \times 49$ FD grid per pad): (a) vibration at L FAB; (b) rotor mode shape. 

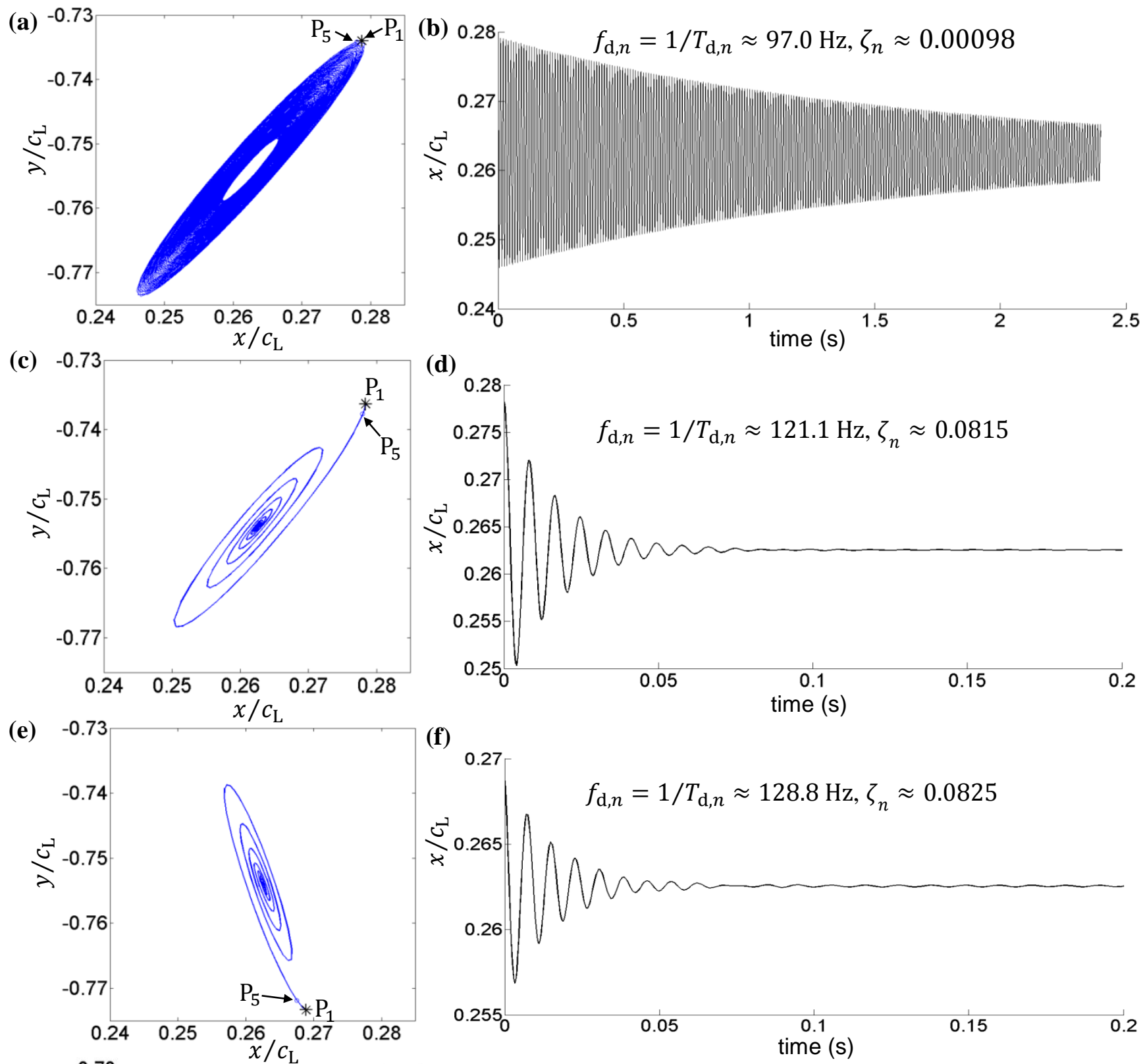

(g)

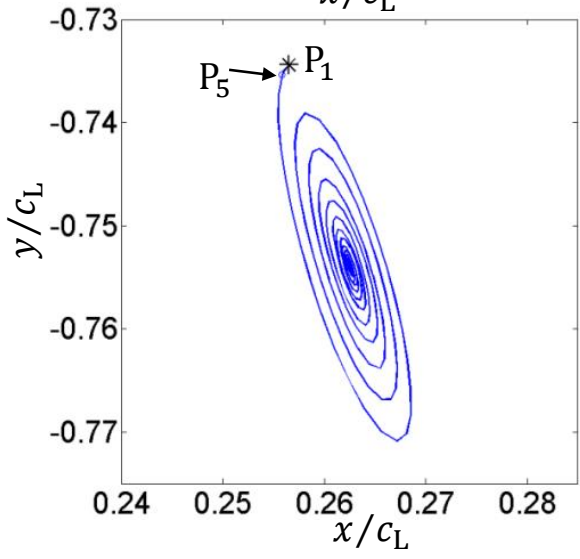

(h)

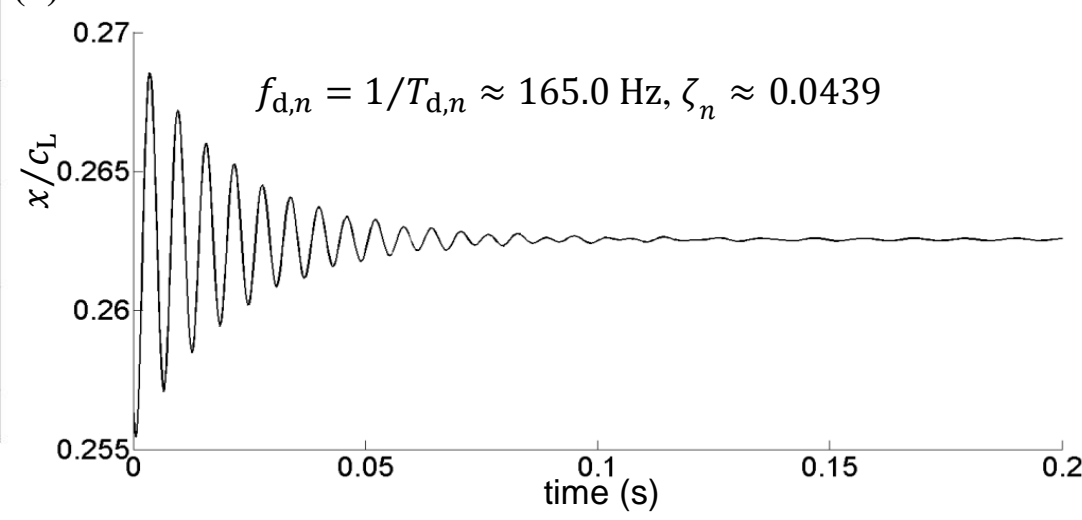

Figure 16. Transient nonlinear dynamic analysis (TNDA) journal trajectories and time histories of L FAB at $30 \mathrm{krpm}$ using mode-specific initial conditions (eq. (54)) taken from Campbell diagram of Figure 10 (Case no. 1, Table 2, with $K_{\mathrm{b}_{\mathrm{L}}}=K_{\mathrm{b}_{\mathrm{R}}}=8.8 \times 10^{9} \mathrm{~N} / \mathrm{m}^{3}$ and $7 \times 49$ FD grid per pad): (a), (b) mode 1 ; (c), (d) mode 2; (e), (f) mode $3 ;(\mathrm{g})$, (h) mode $4\left(\mathrm{P}_{1}\right.$ : point at $t=0 ; \mathrm{P}_{5}$ : point four time steps later; displayed estimates for $f_{\mathrm{d}, n}, \zeta_{n}$ determined from corresponding time histories). 


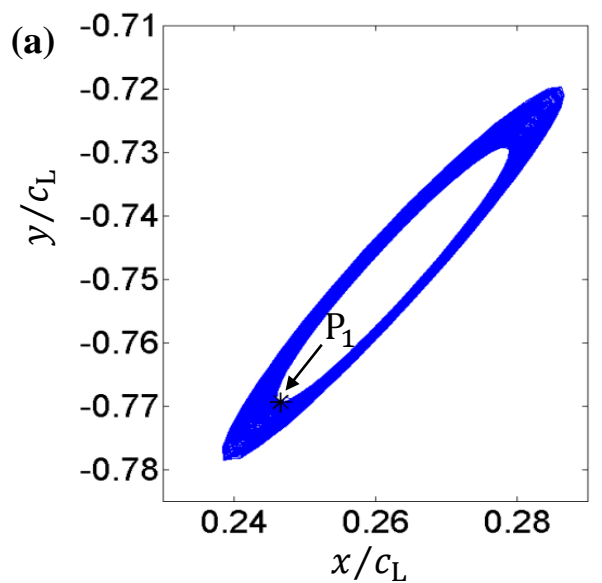

(b)
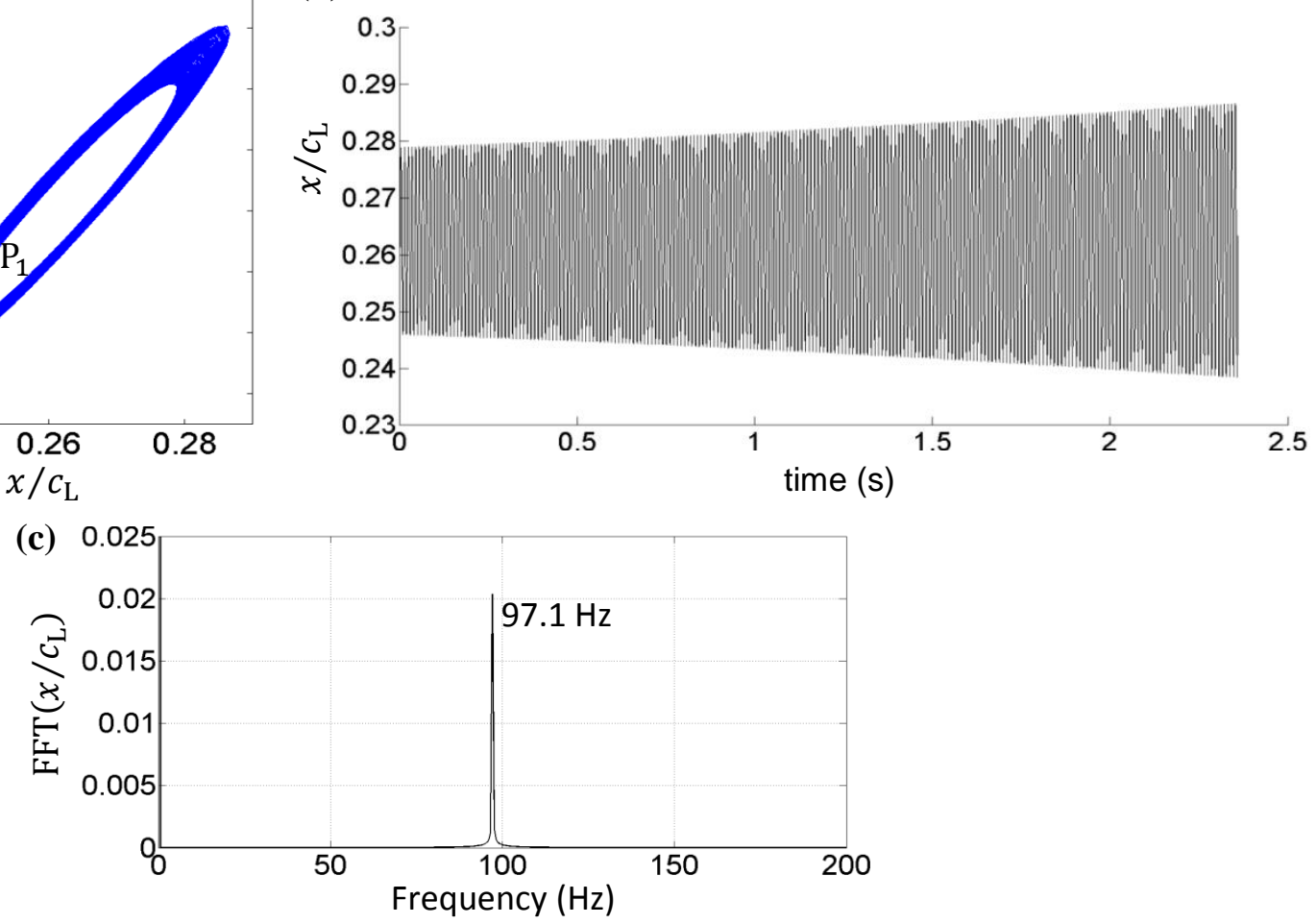

Figure 17. Transient nonlinear dynamic analysis (TNDA) journal trajectory, time history, and frequency spectrum of L FAB at $30.5 \mathrm{krpm}$ using mode-specific (mode 1) initial conditions (eq. (54)) taken from Campbell diagram of Figure 10 (Case no. 1, Table 2, with $K_{\mathrm{b}_{\mathrm{L}}}=K_{\mathrm{b}_{\mathrm{R}}}=8.8 \times 10^{9} \mathrm{~N} / \mathrm{m}^{3}$ and $7 \times 49$ FD grid per pad): (a) journal trajectory; (b) time history; (c) FFT of time history ( $\mathrm{P}_{1}$ : point at $t=0$; for mode 1 @ $30.5 \mathrm{krpm}$ on Campbell diagram, $f_{\mathrm{d}, n}=97.22 \mathrm{~Hz}, \zeta_{n}=-0.0001252$ i.e. unstable). 


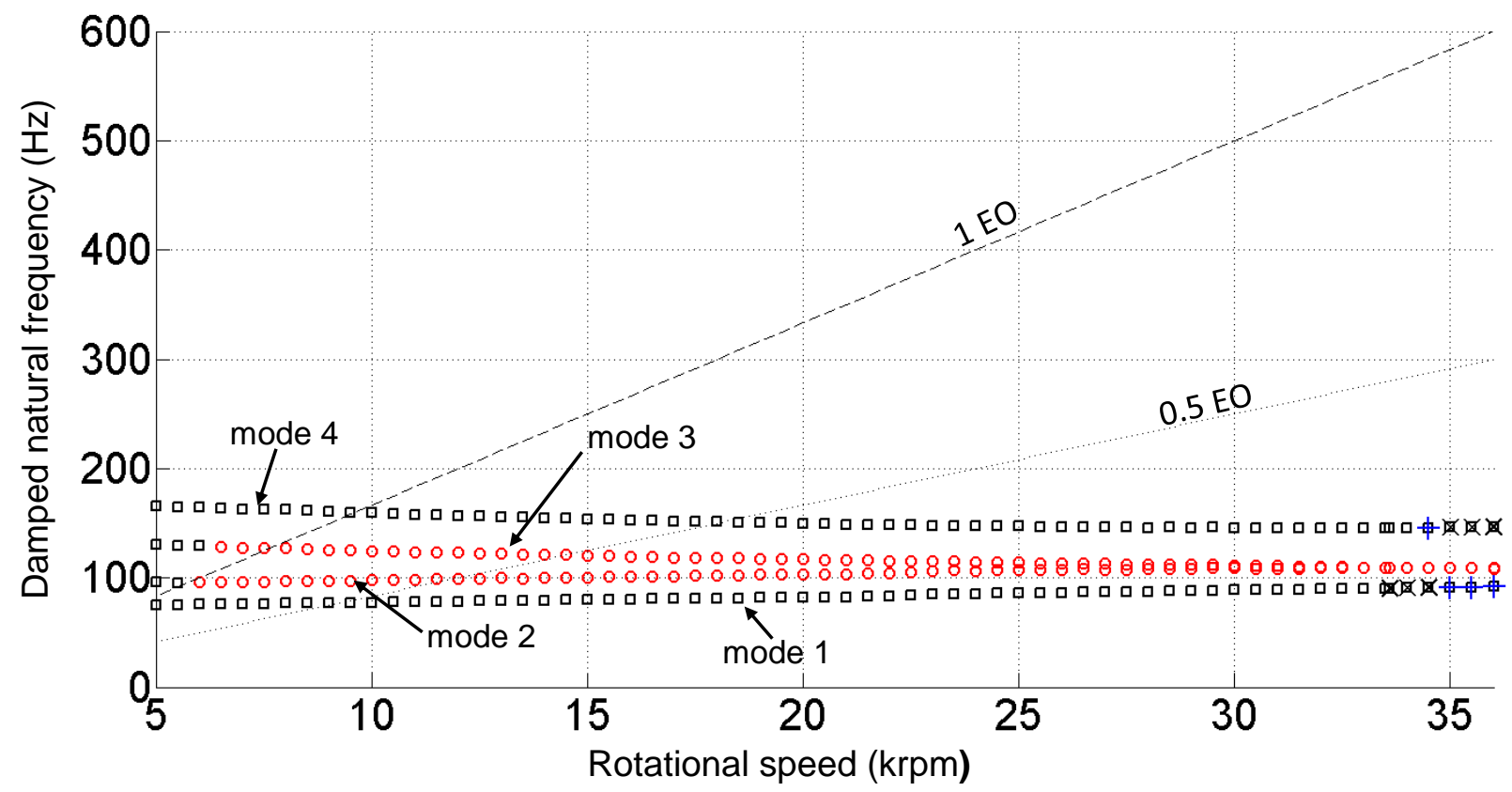

Figure 18. Campbell diagram for Case no. 1 (Table 2) with $K_{\mathrm{b}_{\mathrm{L}}}=K_{\mathrm{b}_{\mathrm{R}}}=4.4 \times 10^{9} \mathrm{~N} / \mathrm{m}^{3}$ and $7 \times 49$ FD grid per pad, extracted from raw eigenfrequency $v s$ speed map using minimum journal amplitude criterion, eq. (51), with $C=0.1$ : forward whirl (black squares); reverse whirl (red circles); unstable mode points overlaid with a cross - oblique black cross for leading unstable mode, upright blue cross for secondary unstable mode; EO (engine order). 


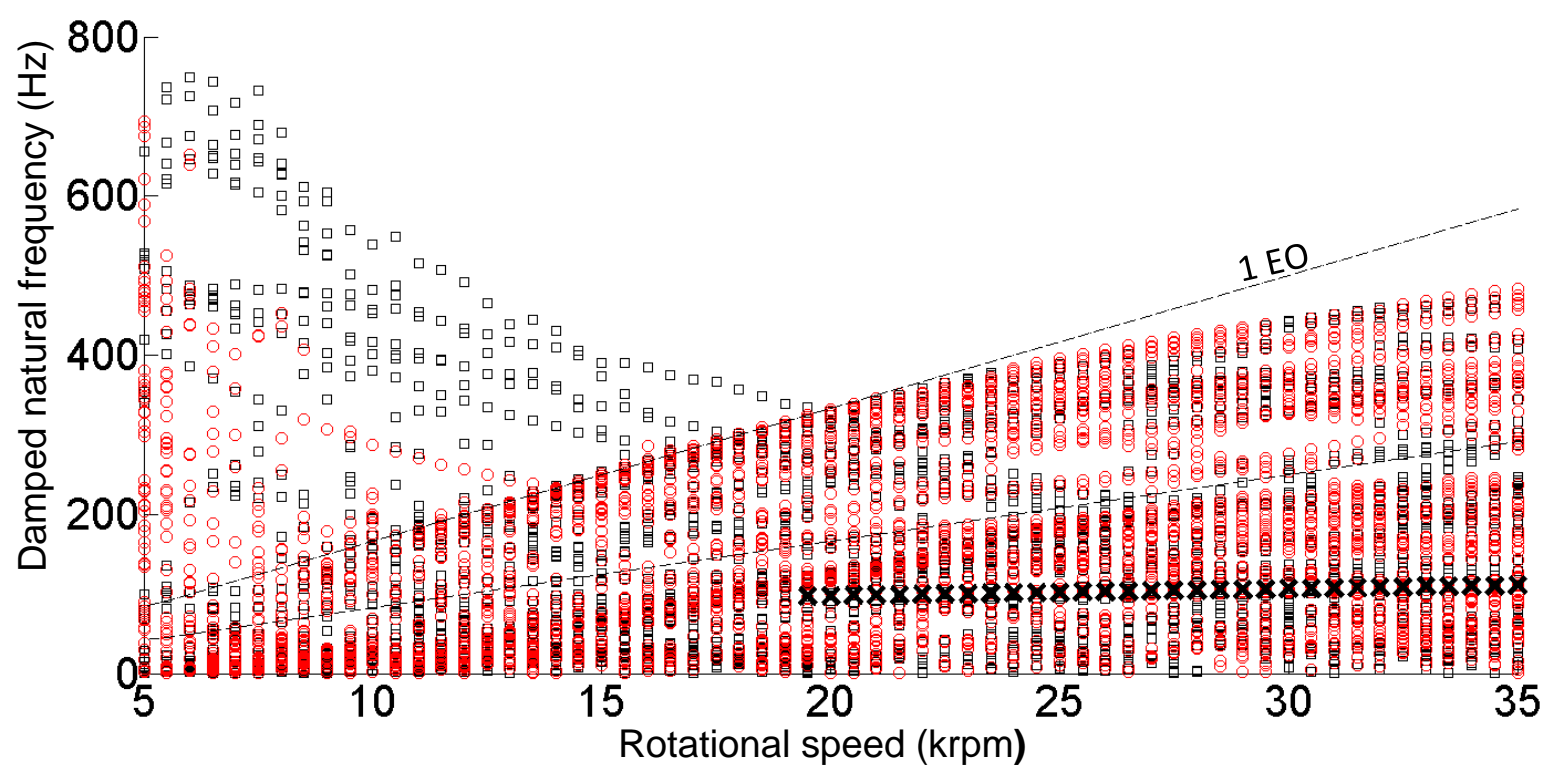

Figure 19. Failed extraction of Campbell diagram from raw eigenfrequency $v s$ speed map using minimum journal amplitude criterion, eq. (51), for Case no. 1 (Table 2) with $K_{\mathrm{b}_{\mathrm{L}}}=K_{\mathrm{b}_{\mathrm{R}}}=8.8 \times$ $10^{16} \mathrm{~N} / \mathrm{m}^{3}$ (i.e. rigid pads) and $7 \times 49$ FD grid per pad: forward whirl (black squares); reverse whirl (red circles); unstable mode points overlaid with a cross; EO (engine order).

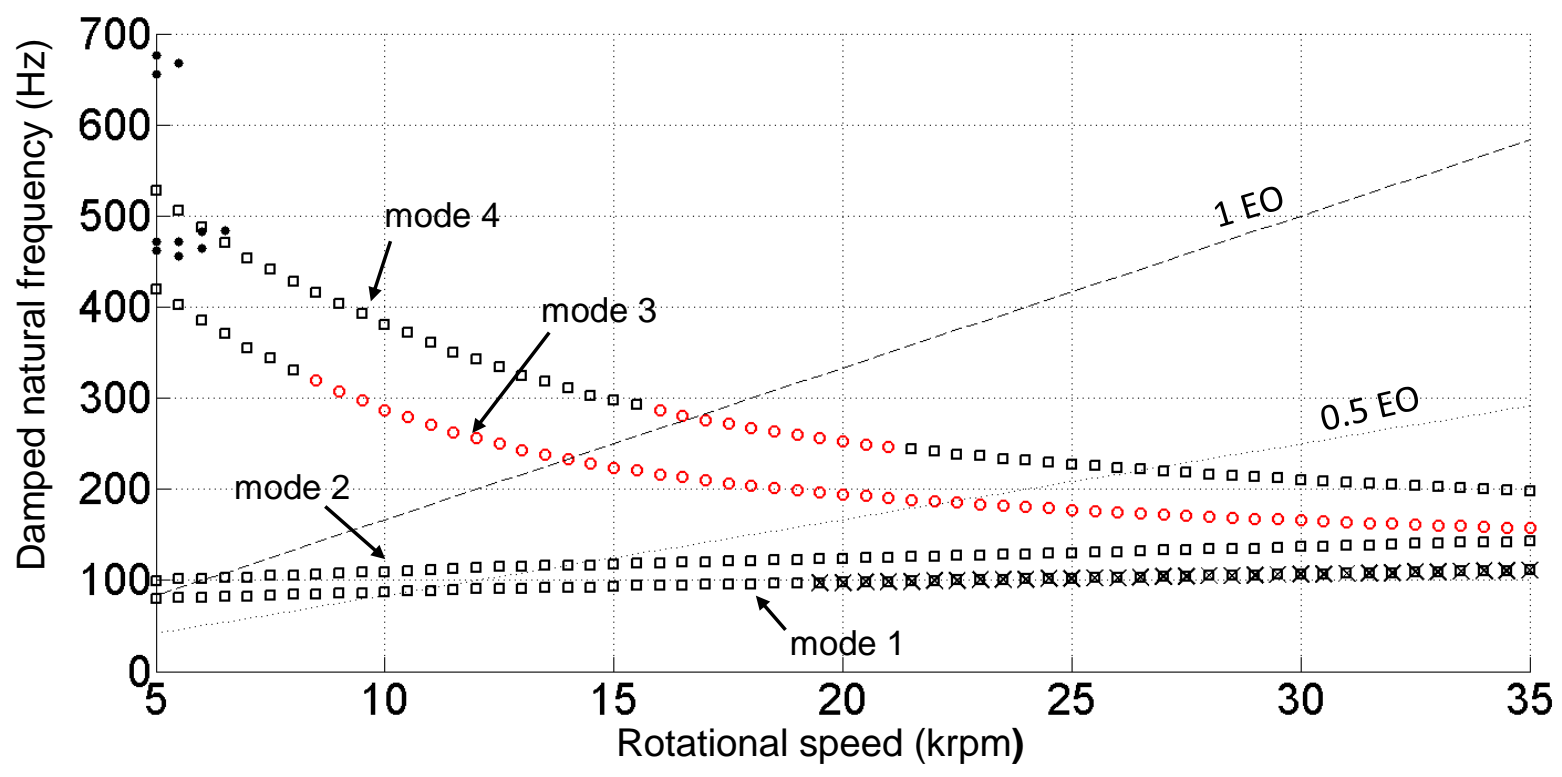

Figure 20. Successful extraction of Campbell diagram from raw eigenfrequency vs speed map using maximum allowable damping ratio criterion of $\zeta_{n}<0.8$ for Case no. 1 (Table 2) with $K_{\mathrm{b}_{\mathrm{L}}}=K_{\mathrm{b}_{\mathrm{R}}}=8.8 \times 10^{16} \mathrm{~N} / \mathrm{m}^{3}$ (i.e. rigid pads) and $7 \times 49$ FD grid per pad: forward whirl (black squares); reverse whirl (red circles); isolated modes (black dots); unstable mode points overlaid with a cross; EO (engine order). 


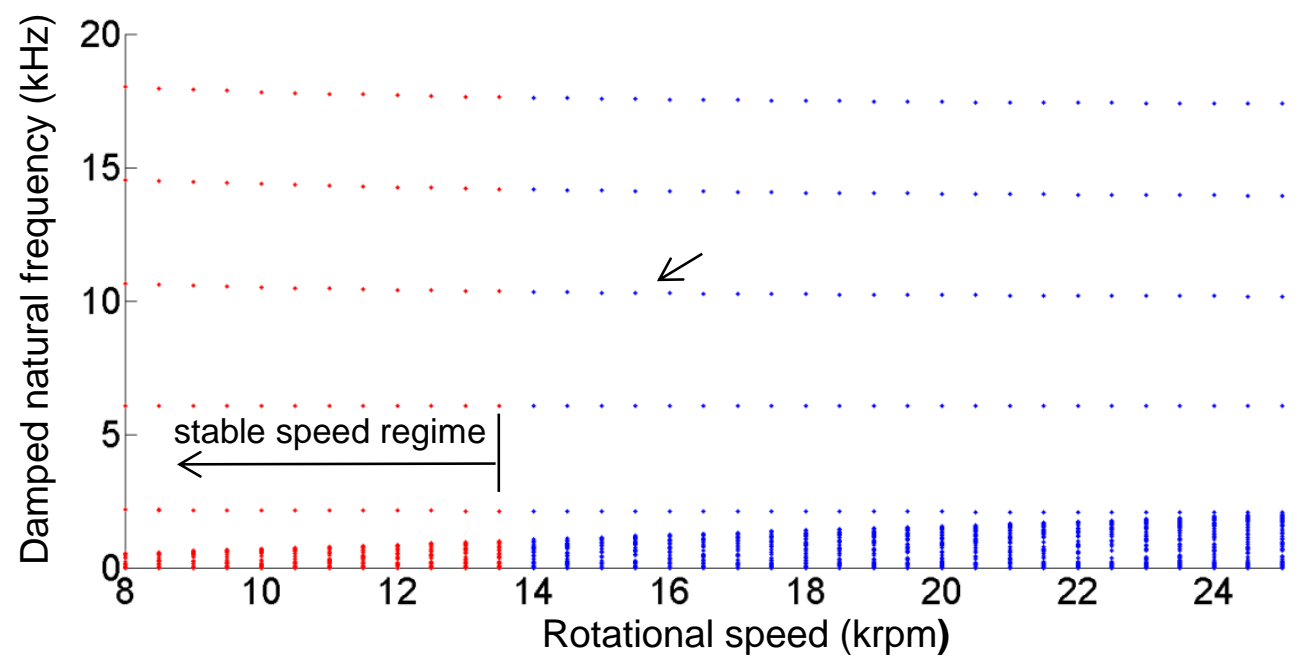

Figure 21. Unfiltered eigenfrequency $\varpi_{\mathrm{d}, n} /(2 \pi) v s$ speed map for Case no. 2 (Table 2) with FD grid is $7 \times 72$ (arrow indicates one of the five high frequency modes introduced by the bump foil inertia).
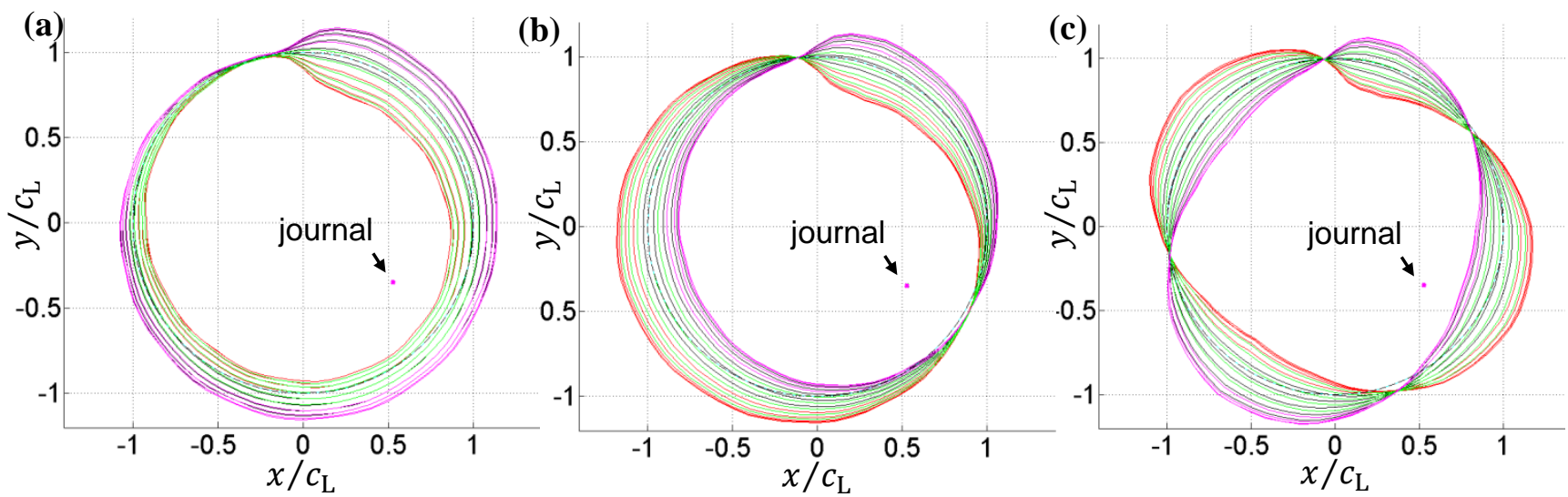

Figure 22. The first, second and fourth of the five high frequency modes introduced by the bump foil inertia, taken from the unfiltered eigenfrequency $v s$ speed map in Figure 21 at $15 \mathrm{krpm}$ : (a) $f_{\mathrm{d}, n}=2.119 \mathrm{kHz}, \zeta_{n}=0.13311$; (b) $f_{\mathrm{d}, n}=6.057 \mathrm{kHz}, \zeta_{n}=0.12957$; (c) $f_{\mathrm{d}, n}=14.138 \mathrm{kHz}$, $\zeta_{n}=0.12175$. 


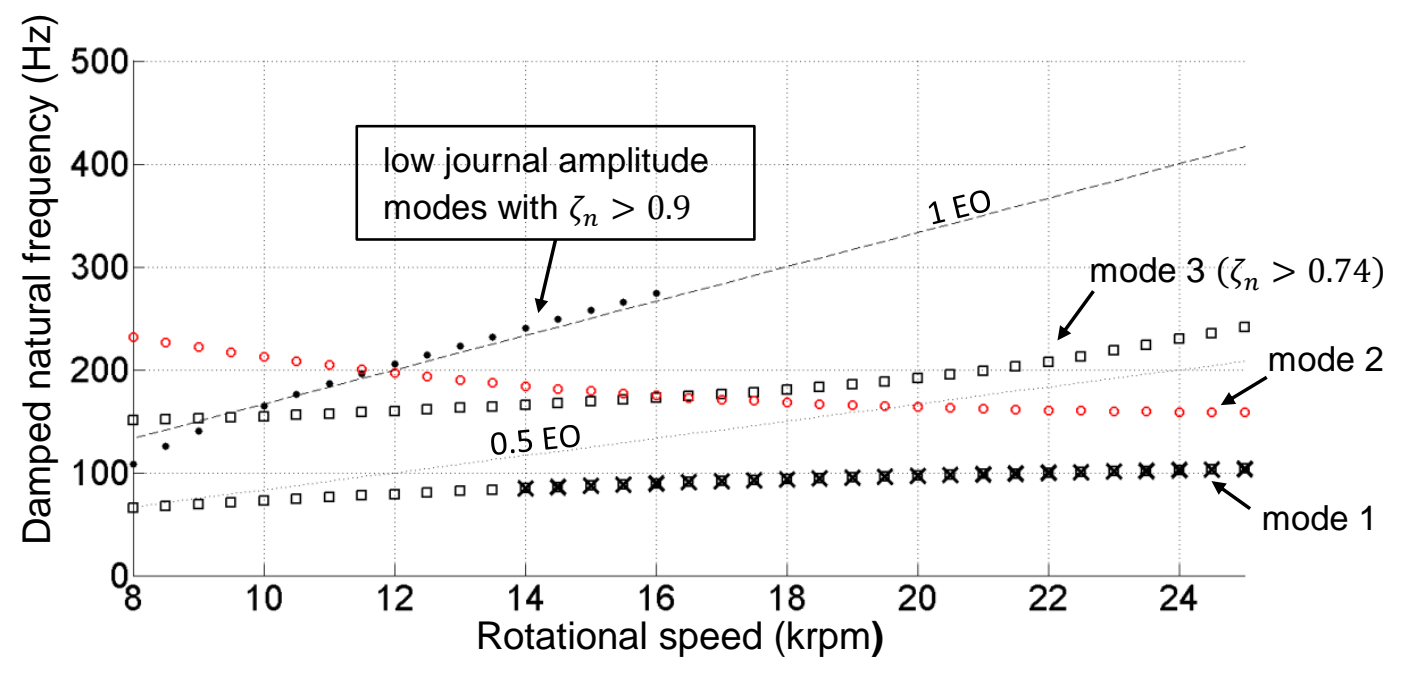

Figure 23. Campbell diagram for Case no. 2 (Table 2) with FD grid is $7 \times 72$, extracted from raw eigenfrequency $v s$ speed map of Figure 21 using minimum journal amplitude criterion, eq. (51), with $C=0.1$ : forward whirl (black squares); reverse whirl (red circles); unstable mode points overlaid with a cross; EO (engine order).

(a)

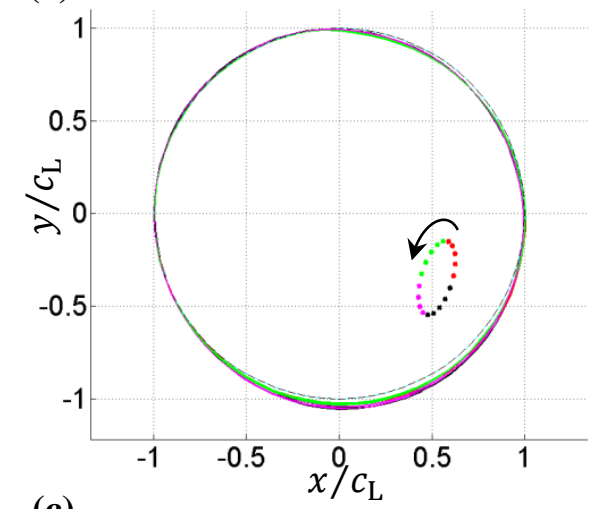

(c)

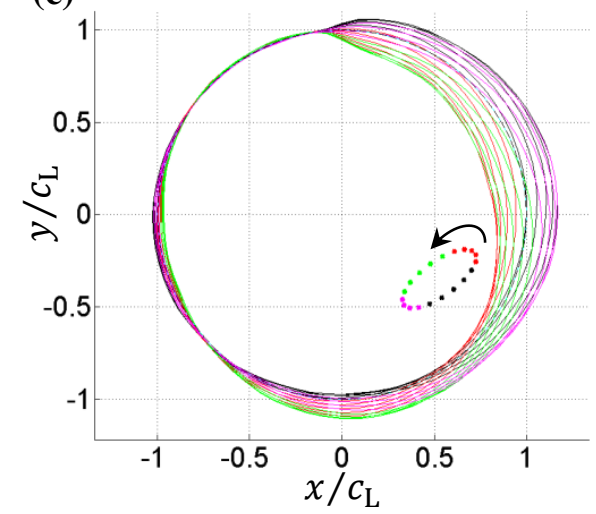

(b)
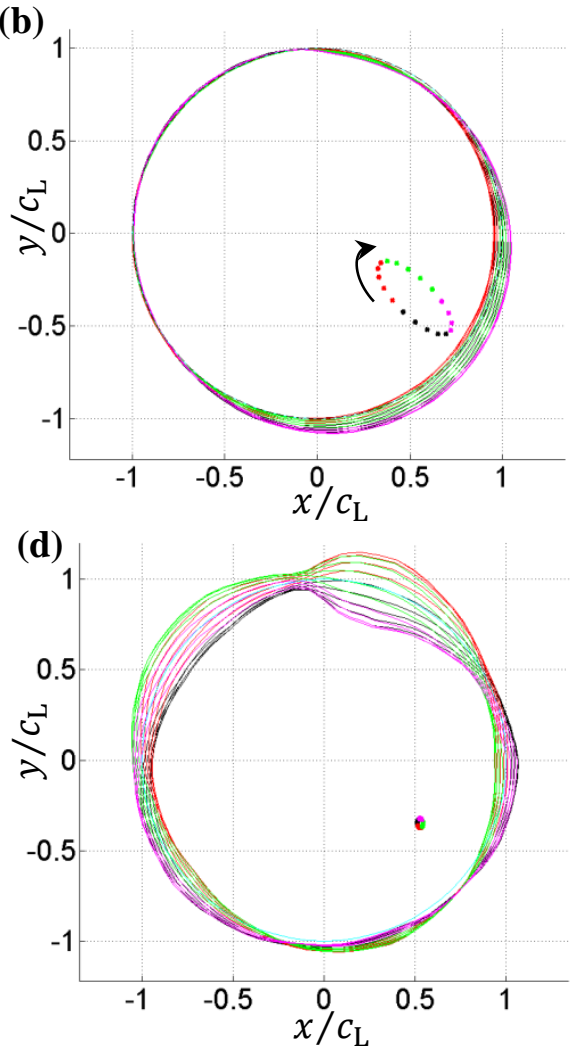

Figure 24. Modes at $15 \mathrm{krpm}$ taken from Campbell diagram in Figure 23: (a) mode $1\left(f_{\mathrm{d}, n}=87.12 \mathrm{~Hz}\right.$, $\zeta_{n}=-0.046431$ i.e. unstable, forward whirl); (b) mode $2\left(f_{\mathrm{d}, n}=179.24 \mathrm{~Hz}, \zeta_{n}=0.28526\right.$, reverse whirl); (c) mode $3\left(f_{\mathrm{d}, n}=168.81 \mathrm{~Hz}, \zeta_{n}=0.86913\right.$, forward whirl) (d) low journal amplitude mode $\left(f_{\mathrm{d}, n}=257.4326 \mathrm{~Hz}, \zeta_{n}=0.96338\right)$. 


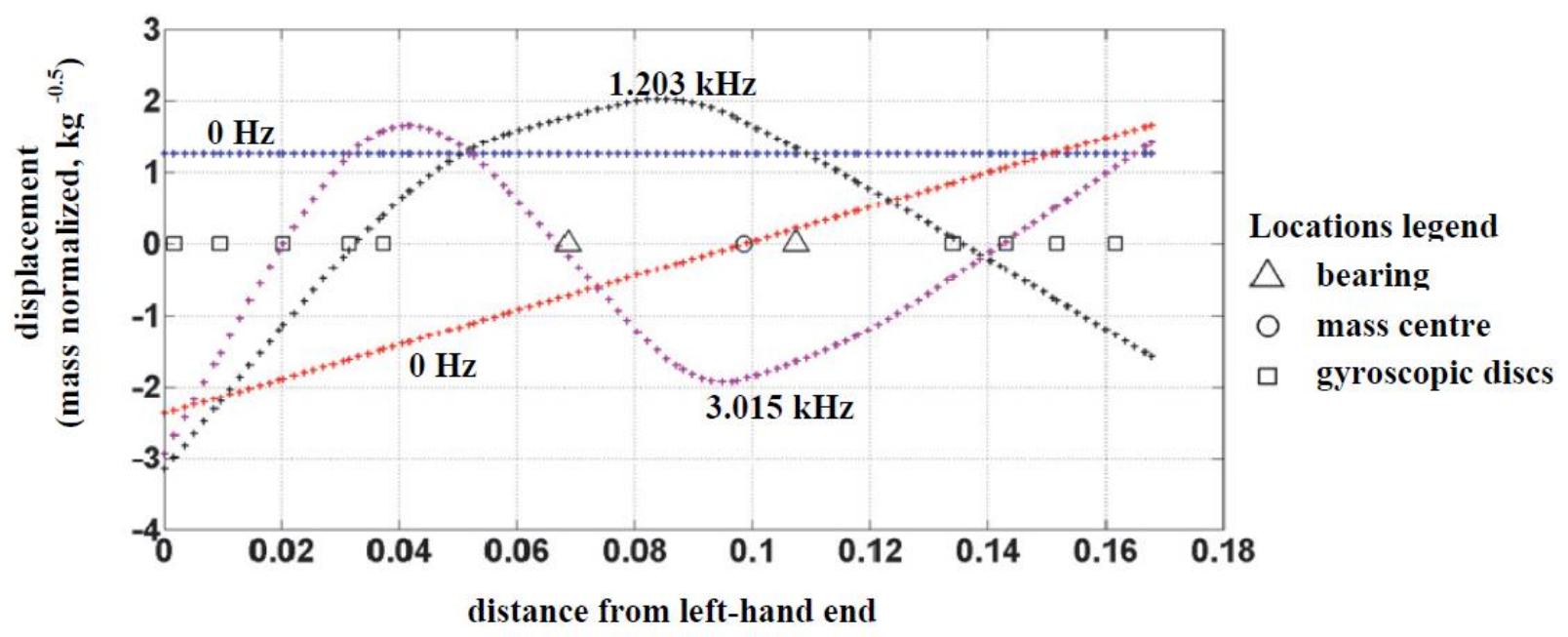

Figure 25. Component modes (free-free undamped rotor modes at zero speed) in $x z$ or $y z$ plane used in Case no. 3 (Table 2) [6, 30]. 


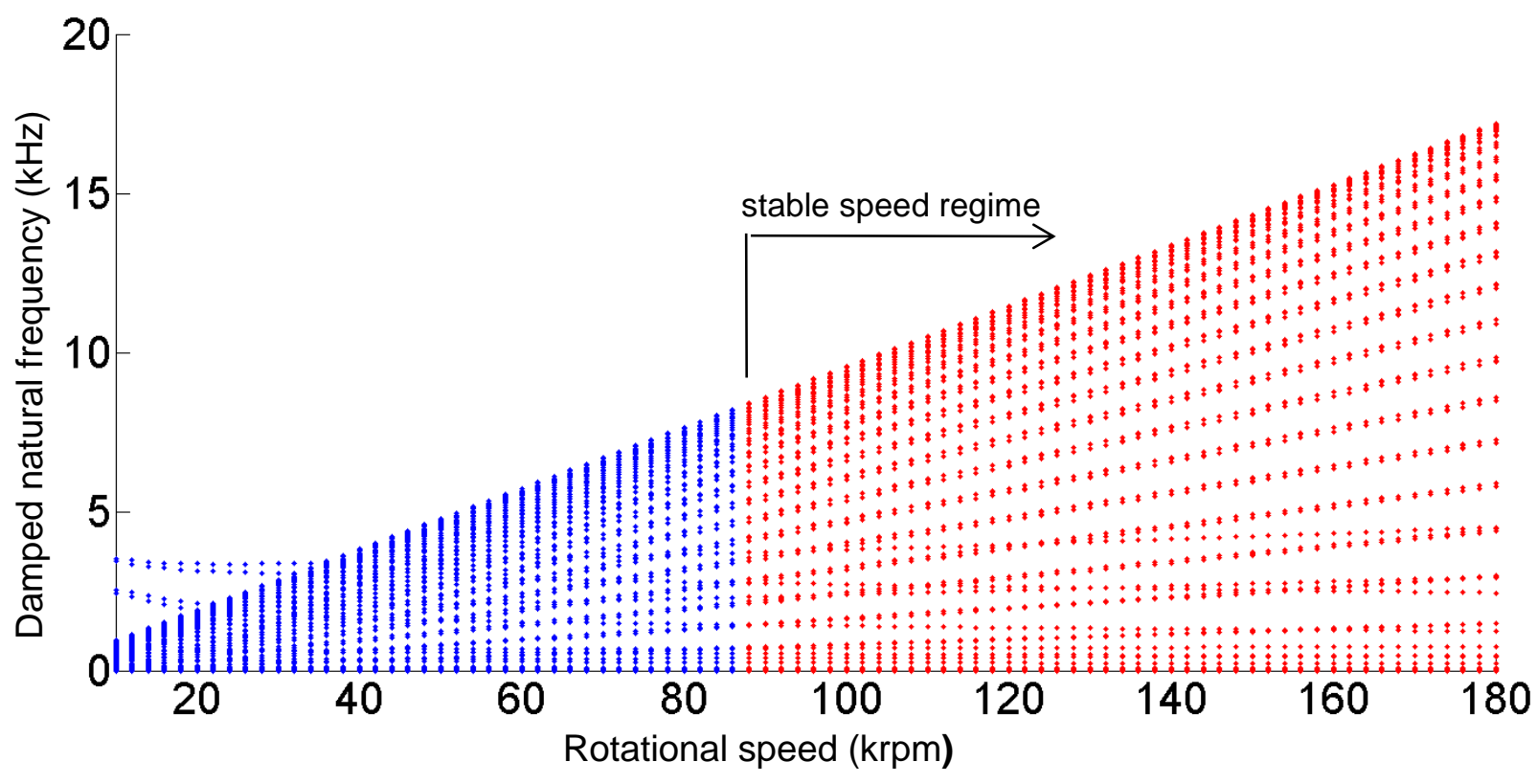

Figure 26. Unfiltered eigenfrequency $\varpi_{\mathrm{d}, n} /(2 \pi) v s$ speed map for Case no. 3 (Table 2) (FD grid is $7 \times 72$ ).

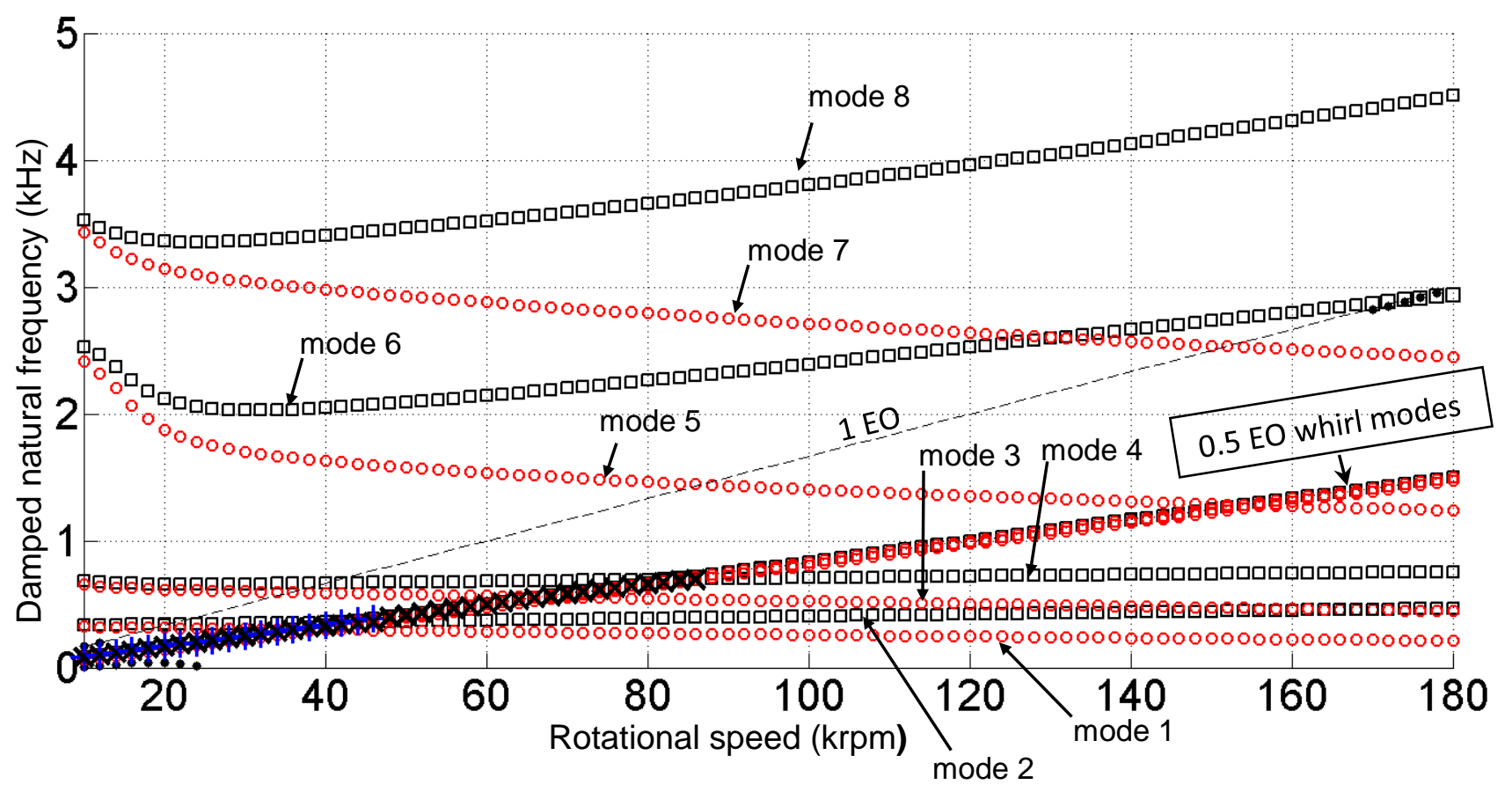

Figure 27. Campbell diagram for Case no. 3 (Table 2) (FD grid is $7 \times 72$ ), extracted from raw eigenfrequency $v s$ speed map of Figure 26 using minimum journal amplitude criterion, eq. (51), with $C=0.1$ : forward whirl (black squares); reverse whirl (red circles); unstable mode points overlaid with a cross - oblique black cross for leading unstable mode, upright blue cross for secondary unstable mode; isolated black dots indicate modes that can be removed by increasing $C$ to 0.25 and adding simultaneous condition $\zeta_{n}<0.7$; EO (engine order). 


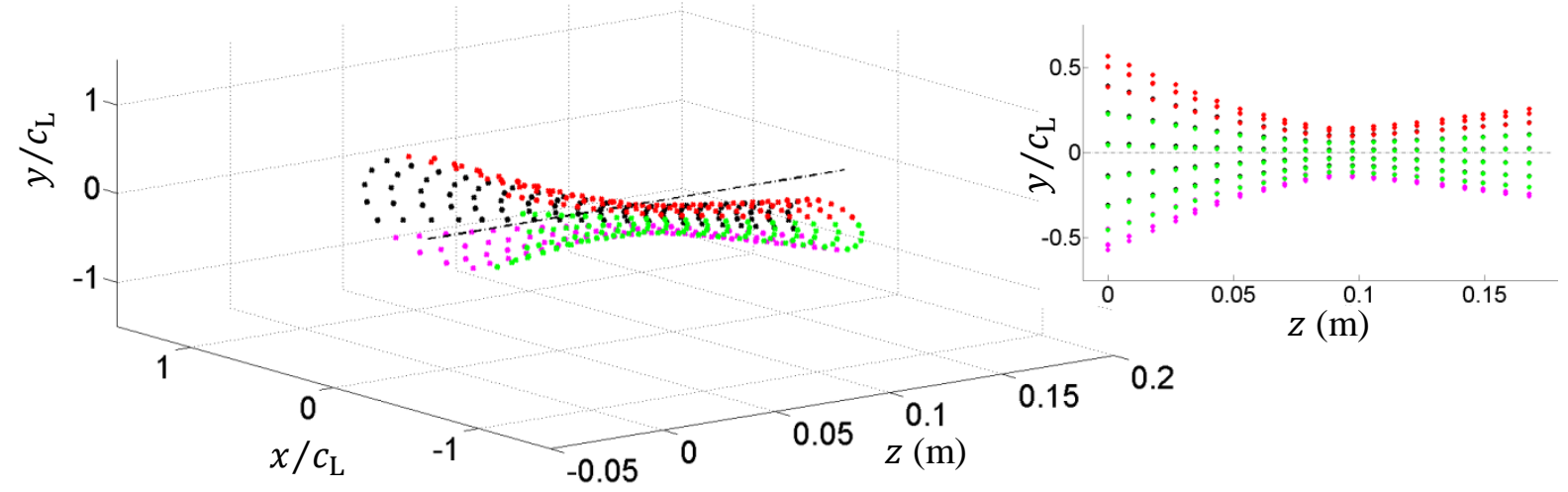

Figure 28. Unstable $0.5 \mathrm{EO}$ whirl mode at $72 \mathrm{krpm}\left(f_{\mathrm{d}, n}=598.65 / \mathrm{Hz}, \zeta_{n}=-0.0052535\right.$, forward whirl), taken from Campbell diagram of Figure 27 (the main figure is the 3D-view including the static equilibrium configuration (SEC) and the inset is the side view with the SEC omitted).
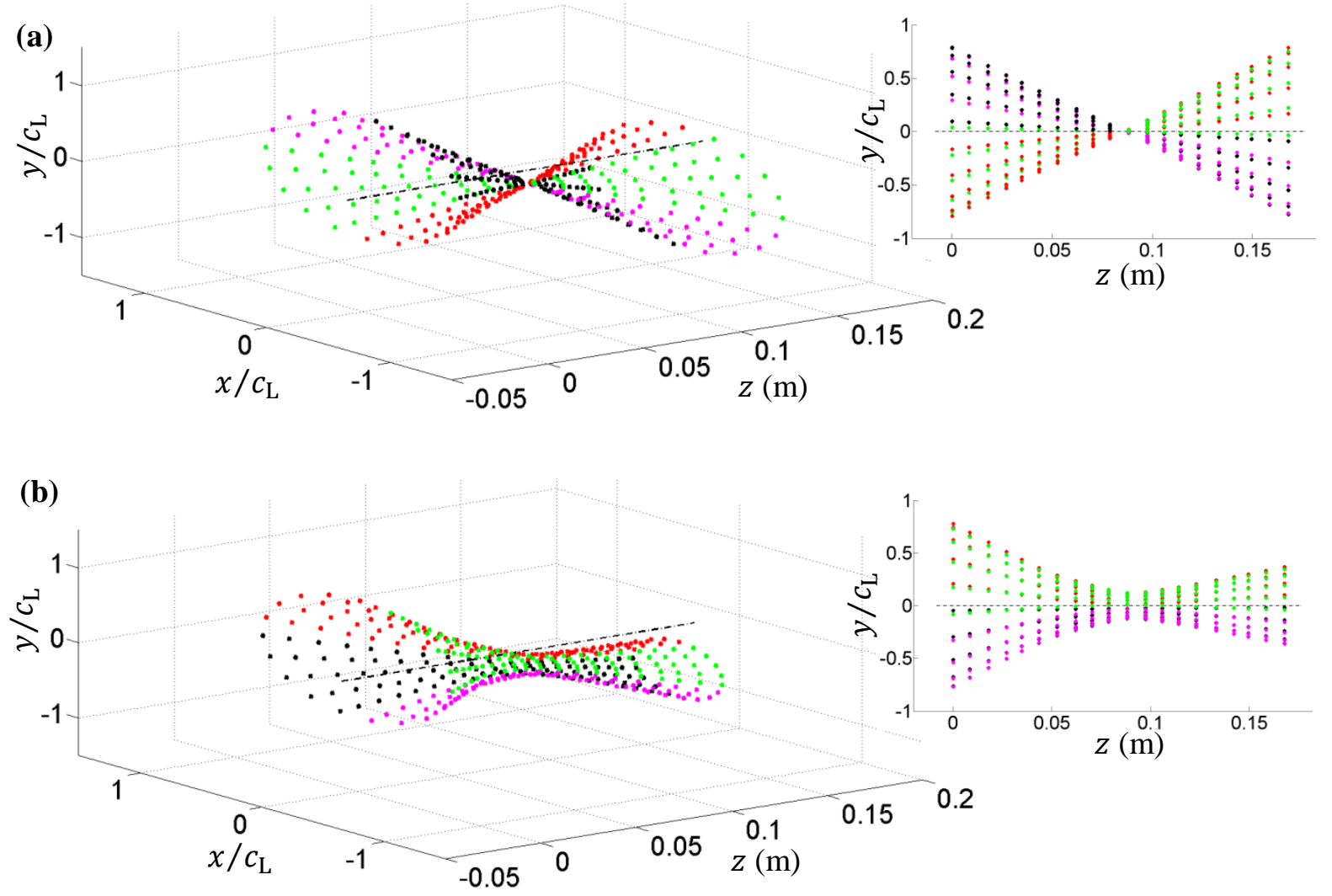

Figure 29. Stable, forward whirl, modes 2, 4 at $72 \mathrm{krpm}$ taken from Campbell diagram of Figure 27: (a) mode $2\left(f_{\mathrm{d}, n}=382.48 / \mathrm{Hz}, \zeta_{n}=0.015414\right)$; (b) mode $4\left(f_{\mathrm{d}, n}=687.33 / \mathrm{Hz}, \zeta_{n}=\right.$ 0.034114 ) (in each case, the main figure is the 3D-view including the static equilibrium configuration (SEC) and the inset is the side view with the SEC omitted). 

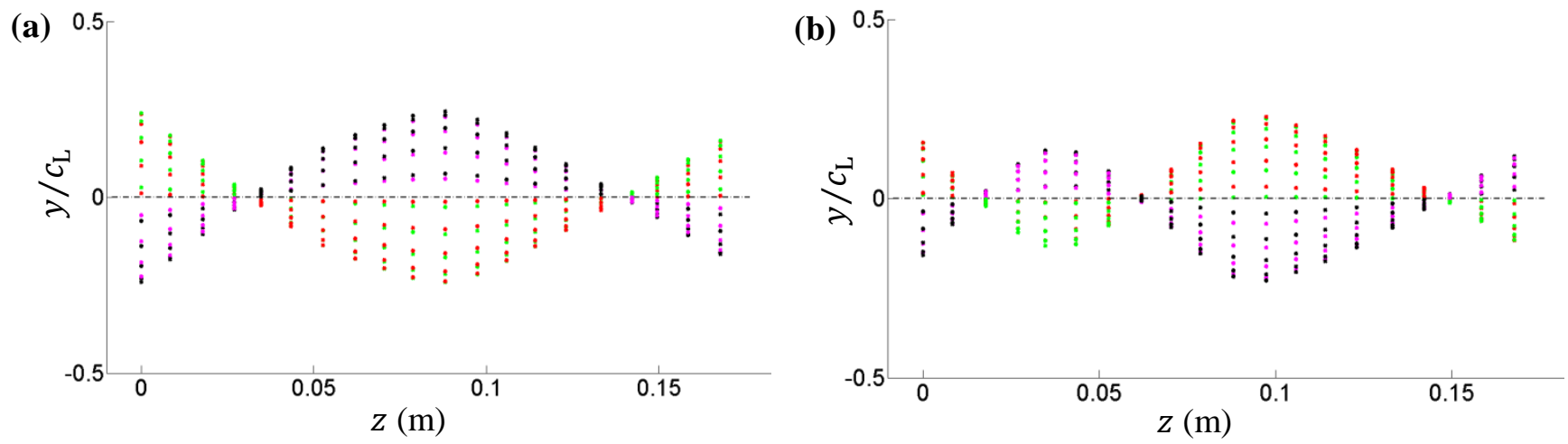

Figure 30. Side view of modes 6 and 7 at speeds where their frequencies intersect with 1 EO line on Campbell diagram of Figure 27: (a) mode 6 at $174 \mathrm{krpm}\left(f_{\mathrm{d}, n}=2.9 / \mathrm{kHz}, \zeta_{n}=0.028722\right.$, forward whirl); (b) mode 7 at $152 \mathrm{krpm}\left(f_{\mathrm{d}, n}=2.5 / \mathrm{kHz}, \zeta_{n}=0.015261\right.$, reverse whirl $)$. NB: static equilibrium configuration omitted. 